\title{
NUMERICAL MODELING OF MICROFLUIDIC TWO-PHASE ELECTROHYDRODYNAMIC INSTABILITY
}

BY VENKAT RAMAN THENKARAI NARAYANAN

\author{
A thesis submitted to the \\ Graduate School-New Brunswick \\ Rutgers, The State University of New Jersey \\ in partial fulfillment of the requirements \\ for the degree of \\ Master of Science \\ Graduate Program in Mechanical and Aerospace Engineering \\ Written under the direction of \\ Professor Hao Lin \\ and approved by
}

New Brunswick, New Jersey

January, 2010 


\section{ABSTRACT OF THE THESIS}

\section{Numerical modeling of microfluidic two-phase electrohydrodynamic instability}

\section{by Venkat raman Thenkarai Narayanan Thesis Director: Professor Hao Lin}

Organic-aqueous liquid (phenol) extraction is one of many standard techniques to efficiently purify DNA directly from cells. Effective dispersion of one fluid phase in the other increases the surface area over which biological component partitioning may occur, and hence enhances DNA extraction efficiency. Electrohydrodynamic (EHD) instability can be harnessed to achieve this goal and has been experimentally demonstrated by Zahn and Reddy (2006). In this work, analysis and simulation are combined to study two-phase EHD instability. In the problem configuration, the organic (phenol) phase flows into the microchannel in parallel with and sandwiched between two aqueous streams, creating a three-layer planar geometry; the two liquid phases are immiscible. An electric field is applied 
to induce instability and to break the organic stream into droplets. The TaylorMelcher leaky-dielectric model is employed to investigate this phenomenon. A linear analysis is carried out with a Chebyshev pseudo-spectral method, whereas a fully nonlinear numerical simulation is implemented using a finite volume, immersed boundary method (IBM). The results from both models compare favorably with each other. The linear analysis reveals basic instability characteristics such as kink and sausage modes. On the other hand, the nonlinear simulation predicts surface deformation in the strongly nonlinear regime pertinent to droplet formation. These numerical tools will be used to investigate the effects of the applied electric field, geometry, and convective flow rate on mixing and dispersion. The eventual objective is to maximize surface area of the organic phase under given experimental conditions for optimized DNA extraction. 


\section{Acknowledgements}

First and foremost, I would like to extend my hearty thanks to my advisor Prof. Hao Lin for his wonderful guidance, motivation and support throughout the course of my Masters research. His thoughtful leadership, meticulous planning and tenacious pursuits towards newer vistas of research were a huge learning experience for a fledging researcher like me. I consider myself extremely fortunate to have been part of his research group. Next, I would like to express my gratitude to Prof. Jeffrey D. Zahn for being my co-advisor and offering me financial support during this research endeavour. Partial support from NSF grants (CBET-0721341, Dr. Zahn) and (CBET-0747886, Dr. Lin) is acknowledged.

My heartfelt gratitude to my current advisor Prof. Joachim V. Heberlein at University of Minnesota for offering me time and computational resources to complete my Masters thesis. I also extend my thanks to Prof. Haim Baruh and Prof. Yogesh Jaluria for offering an admission into the Rutgers MAE department.

I am indebted to Prof. Ellis H. Dill for his immense help during tough times and for being such a wonderful human being.

This thesis work wouldn't have been possible without intense technical discussions with Mr. Jianbo Li. My sincere thanks for his enthusiasm and suggestions. My colleague Mr. Jia Zhang has been extremely helpful during my stay. My 
roommates Hari, Shiva and Raghu shared invaluable moments to help me beat the stress out of research and academics. My friends Pappu, Pallab, Sai, Jingru, Kellie, Tushar, Dhaval and Murthy have been brave, caring and patient during their interactions with me. I'd cherish our group's sweet moments during lunch sessions and New Jersey Symphony Orchestra's performances.

Last but not the least, my Dad and Grandma have been the bedrock of support throughout my life. Words of gratitude aren't enough for their love and sacrifices. 


\section{Dedication}

To my Appa and Paatti 


\section{Table of Contents}

Abstract .......................... ii

Acknowledgements ................... iv

Dedication ............................... vi

List of Tables . . . . . . . . . . . . . . . . . .

List of Figures . . . . . . . . . . . . . . . . xi

1. Introduction . . . . . . . . . . . . . . . 1

1.1. Electrohydrodynamics in microchannel flows . . . . . . . . . . . 1

1.2. Motivation: Organic-aqueous extraction ........... 3

1.3. Previous work . . . . . . . . . . . . . . . . 5

1.4. Issues and challenges . . . . . . . . . . . . . . . . 11

1.5. Scope of the thesis . . . . . . . . . . . . . . . 11

2. Linear stability analysis . . . . . . . . . . . . . . 13

2.1. Problem specification ................... 13

2.2. Governing equations ................... . . 14

2.3. Numerical solution via pseudospectral methods . . . . . . . . . . 17

2.3.1. Scaling and dimensionless parameters . . . . . . . . . 18 
2.3.2. Dimensionless governing equations . . . . . . . . . . . 19

2.3.3. Perturbation analysis . . . . . . . . . . . . . . . . . 19

2.3.4. Chebyshev pseudospectral method . . . . . . . . . 28

2.4. Results from pseudospectral method . . . . . . . . . . . . . . 30

2.4.1. Effects of electrical property ratios . . . . . . . . . 31

2.4.2. Effects of depth ratio . . . . . . . . . . . . . . 38

2.4.3. Effects of electric field . . . . . . . . . . . . . . 39

2.4.4. Effects of surface tension . . . . . . . . . . . . . 42

2.4.5. Effects of convective base flow . . . . . . . . . . . . 43

3. Fully nonlinear simulation . . . . . . . . . . . . . . . . 45

3.1. Nonlinear simulation methodology . . . . . . . . . . . . . . . 45

3.1.1. Governing equations ............... 45

3.1.2. Scaling and dimensionless parameters . . . . . . . . . . 47

3.1.3. Numerical solution technique . . . . . . . . . . . . . . 47

3.1.4. Staggered grid . . . . . . . . . . . . . . . . 49

3.1.5. Interface tracking . . . . . . . . . . . . . 51

3.1.6. Flow solver ................. . . 53

3.2. Validation .................... 63

3.2.1. Step size accuracy . . . . . . . . . . . . . . . . 63

3.2.2. Growth rate calculation accuracy ........... 64

3.2.3. Grid resolution validation ............. 65 
3.2.4. Miscellaneous validations . . . . . . . . . . . . . . . 67

3.3. Results from nonlinear analysis . . . . . . . . . . . . . . 68

3.3.1. Electrical conductivity variation . . . . . . . . . 70

3.3.2. Electrical conductivity and density variations . . . . . 72

3.3.3. Electrical conductivity, density and permittivity variations 73

3.3.4. Electrical conductivity, density, permittivity and viscosity variations . . . . . . . . . . . . . . . 75

3.3.5. Grid resolution results . . . . . . . . . . . . . 79

4. Conclusions and directions of future work . . . . . . . . . . . 81

4.1. Summary of the thesis . . . . . . . . . . . . . 81

4.2. Future directions .................... 85

Appendix A. Long-wave asymptotic analysis . . . . . . . . . . 87

A.1. Scaling and dimensionless parameters . . . . . . . . . . . 87

A.2. Dimensionless governing equations . . . . . . . . . . 88

A.3. Perturbation analysis . . . . . . . . . . . . . . 93

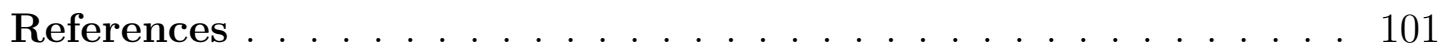




\section{List of Tables}

3.1. Summary of growth rate results comparison between linear and nonlinear analyses for the four different cases. . . . . . . . . . . 79

A.1. Various scales used in the long-wave analysis. . . . . . . . . . . 88 


\section{List of Figures}

1.1. EHD instability experiments in a two fluid interface [15]. Interfacial deformation, rupture and subsequent droplet formation can be observed. . . . . . . . . . . . . . . . . 3

1.2. Schematic of the set-up for EHD instability in a three-layer stratified flow [3]. The electric field is applied perpendicular to the direction of flow. . . . . . . . . . . . . . . . . 4

1.3. EHD instability experiments in a three-layer stratified flow [3]. The formation of droplets and enhanced mixing as electric field is increased can be observed. . . . . . . . . . . . . . . . 5

2.1. Two dimensional domain for linear analysis of three-layer viscous stratified flow. The schematic shows the electric field applied parallel to the conductivity gradient. . . . . . . . . . . . . . 14 
2.2. The plot of imaginary part of dimensional growth rate $(\operatorname{Im}(\omega))$ vs. the dimensional wavenumber $(\mathrm{kx})$ for cases A and B (Type-I) for which the product $(\sigma-1)\left(\sigma^{2}-\epsilon\right)$ is positive. Higher growth rates for both modes compared to cases C and D (Type-II) are predicted primarily because the electrical properties considered have a destabilizing effect on the flow. In figure (a), $\sigma=10^{1}, \epsilon=10^{1}$ and in figure (b) $\sigma=10^{-0.5}, \epsilon=10^{1}$ have been used. . . . . . . . . . . .

2.3. The plot of imaginary part of dimensional growth rate $(\operatorname{Im}(\omega))$ vs. the dimensional wavenumber $(\mathrm{kx})$ for cases $\mathrm{C}$ and $\mathrm{D}$ (Type-II) for which the product $(\sigma-1)\left(\sigma^{2}-\epsilon\right)$ is negative. Lower growth rates for both modes compared to cases A and B (Type-I, figure 2.2) can be observed. The electrical properties considered are said to have a stabilizing effect on the flow. In figure (a) $\sigma=10^{-0.5}, \epsilon=10^{-1.5}$ and in figure (b) $\sigma=10^{0.25}, \epsilon=10^{1}$ are used. . . . . . . . . . . 34

2.4. The plot of imaginary part of dimensional growth rate $(\operatorname{Im}(\omega))$ vs. the dimensional wavenumber $(\mathrm{kx})$ for cases $\mathrm{E}$ (Type-III) for which the product $(\sigma-1)\left(\sigma^{2}-\epsilon\right)$ is zero. The stability/instability of the flow is dictated by the tangential stress contributions $\left(Q_{T}\right)_{1}$ and $\left(Q_{T}\right)_{2}$ alone. The values of $\sigma=10^{0.5}, \epsilon=10^{1}$ are used. . . . . . 34 
2.5. Log-log plot of electrical conductivity ratio $(\sigma)$ against dielectric permittivity ratio $(\epsilon)$ depicting maximum growth rate values for the case of kink mode with the depth ratio $n=1$. The neutral stability curve (red) and other constant growth rate curves have been plotted. Three dashed lines corresponding to $\epsilon=\sigma, \epsilon=\sigma^{2}$ and $\sigma=1$ are also drawn to highlight the regions pertaining to types I and II. . . . . . . . . . . . . . . .

2.6. Log-log plot of electrical conductivity ratio $(\sigma)$ against dielectric permittivity ratio $(\epsilon)$ with maximum growth rate values for the case of sausage mode with the depth ratio $n=1$. The neutral stability curve (red), a few constant growth rate curves and the three dashed lines corresponding to $\epsilon=\sigma, \epsilon=\sigma^{2}$ and $\sigma=1$ are plotted. . . . . 36

2.7. The plot of imaginary part of dimensional growth rate $(\operatorname{Im}(\omega))$ vs. the dimensional wavenumber $(\mathrm{kx})$ for cases A and B (Type-I) and the depth ratio $n$ equals 0.5 . Higher growth rates for both modes compared to cases C and D (Type-II) are predicted as the electrical properties tend to destabilize the flow. In figure (a), $\sigma=10^{1}, \epsilon=10^{1}$ and in figure (b) $\sigma=10^{-0.5}, \epsilon=10^{1} \ldots \ldots 38$ 
2.8. The plot of imaginary part of dimensional growth rate $(\operatorname{Im}(\omega))$ vs. the dimensional wavenumber $(\mathrm{kx})$ for cases $\mathrm{C}$ and D (Type-II) and the depth ratio $n$ equals 0.5 . Lower growth rates for both modes compared to cases A and B (Type-I, figure 2.2) can be observed. The electrical properties are said to have a stabilizing effect on the flow. In figure (a) $\sigma=10^{-0.5}, \epsilon=10^{-1.5}$ and in figure (b) $\sigma=10^{0.25}, \epsilon=10^{1}$ are used. . . . . . . . . . . . .

2.9. The growth rate plot for kink mode perturbation pertaining to cases $A$ and $D$ with various electric field values. The specific values of $\sigma=10^{1}, \epsilon=10^{1}$ and $\sigma=10^{0.25}, \epsilon=10^{1}$ are taken for figures (a) and (b) respectively. With increasing electric field, flow tends to be more unstable with higher growth rates for case A. An opposite effect is observed for case D. . . . . . . . . . . . .

2.10. The growth rate plot for sausage mode perturbation pertaining to cases $\mathrm{A}$ and $\mathrm{D}$ with various electric field values. Same parametric values as figure 2.9 are used. An anologous behaviour as in the case of kink mode (figure 2.9) has been observed except that for case D, growth rates increase with increase in electric field for low

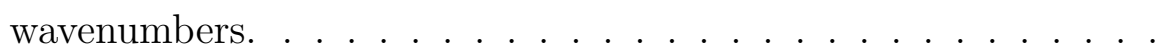


2.11. The growth rate plot for case A and various surface tension coefficients. The decrease in surface tension results in failure to stabilize the waves with high wavenumber (or small wavelength) resulting in higher growth rate values and a wider range of fastest growth rate wavenumbers. This trend has been observed for both kink (figure (a)) and sausage (figure (b)) modes. The values of $\sigma=10^{1}$ and $\epsilon=10^{1}$ were used. . . . . . . . . . . . . . . . .

2.12. The growth rate plot for kink mode and various superposed baseflow values (user-defined parameter $U_{c}$ ). Long wave growth is suppressed while short wave growth rates increase with increase in the user-defined parameter $U_{c}$ (pressure gradient). The fastest growth rate wavenumbers increase correspondingly. For case A, $\sigma=10^{1}$ and $\epsilon=10^{1}$ were used while for case D, $\sigma=10^{0.25}$ and $\epsilon=10^{1}$ were used. . . . . . . . . . . . . . . . . . .

2.13. The growth rate plot for sausage mode and various superposed base-flow values (user-defined parameter $U_{c}$ ). Same parametric ratios as figure 2.12 are considered. Similar conclusion as that for the kink mode applies for the sausage mode. . . . . . . . . . . . . 44

3.1. control volume for $\mathrm{u}, E_{x} \ldots \ldots \ldots$. . . . . . . . 50

3.2. control volume for $\mathrm{v}, E_{y} \ldots \ldots \ldots \ldots$. . . . . . . . 51

3.3. control volume for $p, \sigma, \mu, \epsilon, \rho$ and $\Phi \ldots \ldots . \ldots . \ldots 52$ 
3.4. Log-log plot for error norm variation with time-step size for (a) $\sigma$ variation alone and (b) $\sigma$ and $\rho$ variations. The slope values obtained are (a) 0.8123 and (b) 0.7528 respectively, meaning that the algorithm is approximately first-oder accurate. . . . . . .

3.5. Semi-log plot of maximum interface displacement with time for (a) $\sigma$ variation alone and (b) $\sigma$ and $\rho$ variations for three different time-step sizes. With respect to the baseline values obtained for $\Delta t=5 \times 10^{-4}$, the maximum error in the growth rate calculation for cases (a) and (b) are $0.15 \%$ and $0.11 \%$ respectively. . . . . . . 65

3.6. Growth rates extracted from nonlinear simulation for $k^{*}=10.0$, sausage mode for a conductivity ratio of 100 , density ratio of 0.752 , permittivity ratio of 10.974 , viscosity ratio of 0.284 . The extracted growth rate for double resolution (DR) case is $3.331 \times 10^{4} s^{-1}$, a $2.67 \%$ difference from the growth rate result obtained from the original resolution (OR) dataset captured in the same time interval. 66

3.7. Electrical conductivity profile evolution for the case of conductivity ratio 100 and all other property ratios equal to 1 . The (a) initial profile, profiles at (c) $25 \mu s$ and (e) $30 \mu s$ for conductivity are shown. Red denotes the high conductivity regions (aqueous phase), and blue, the low conductivity region (phenol). (b), (d) and (f) depict the charge density snapshots at corresponding times. . . . 71 
3.8. Growth rates extracted from nonlinear simulation for conductivity variation alone with $k^{*}=10.0$, sausage mode. The ' $\times$ ' are from the simulation with a dimensionless time step size of $\Delta t=5 \times 10^{-4}$, and the line is from least-square (LS) fitting, from which the growth rates are calculated. The extracted growth rate is $1.877 \times 10^{5} \mathrm{~s}^{-1}$, an $8.25 \%$ difference from the linear analysis result. . . . . . . . . 72

3.9. Interface evolution for the case of conductivity ratio 100, density ratio 0.752 and all other property ratios equal to 1 . The profiles at (b) $26 \mu \mathrm{s}$ and (c) $30 \mu \mathrm{s}$ are shown. Red denotes the high density region (phenol), and blue, the low density regions (aqueous phase). 73

3.10. Growth rates extracted from nonlinear simulation with $k^{*}=10.0$, sausage mode for a conductivity ratio 100 and density ratio 0.752 . The ' + ' are from the simulation with a dimensionless time step size of $\Delta t=5 \times 10^{-4}$. The extracted growth rate is $1.615 \times 10^{5} s^{-1}$, a $0.35 \%$ difference from the linear analysis result. . . . . . . . . . 74

3.11. Interface evolution for the case of conductivity ratio 100, density ratio 0.752 , permittivity ratio 10.974 and viscosity ratio 1 . The profiles at (a) $95 \mu s$ and (c) $125 \mu s$ for permittivity are shown. Red denotes the high permittivity regions (aqueous phase), and blue, the low permittivity region (phenol). The corresponding charge density snapshots are shown in (b) and (d) respectively. . . . . . 75 
3.12. Growth rates extracted from nonlinear simulation for $k^{*}=10.0$, kink mode for a conductivity ratio 100 , density ratio 0.752 and permittivity ratio 10.974 . The ' $\square$ ' are from the simulation with a dimensionless time step size of $\Delta t=5 \times 10^{-4}$. The extracted growth rate is $4.602 \times 10^{4} s^{-1}$, a $14.90 \%$ difference from the linear analysis result. 76

3.13. Interface evolution for the case of conductivity ratio 100, density ratio 0.752 , permittivity ratio 10.974 and viscosity ratio 0.284 with an initial sausage-mode perturbation of $k^{*}=10.0$. The profiles at (a) $75 \mu \mathrm{s}$ and (b) $150 \mu \mathrm{s}$ are shown. Red denotes the high viscosity region (phenol), and blue, the low viscosity regions (aqueous phase). 77

3.14. Growth rates extracted from nonlinear simulation for $k^{*}=10.0$, sausage mode for a conductivity ratio 100 , density ratio 0.752 , permittivity ratio 10.974 and viscosity ratio 0.284 . The ' $\nabla$ ' are from the simulation with a dimensionless time step size of $\Delta t=5 \times 10^{-4}$. The extracted growth rate is $3.298 \times 10^{4} s^{-1}$, a $9.70 \%$ difference from the linear analysis result. . . . . . . . . . . . . . . . 78

3.15. Interface profile evolution snapshots at (a) $270 \mu \mathrm{s}$ and (b) 320 $\mu s$ for the interfaces initialized with small-amplitude white-noise disturbance and properties mentioned in figure 3.13. The profiles exhibit mixed unstable modes of different wavenumbers as observed in experiments of Zahn and Reddy [3]. . . . . . . . . . . . . . . . 79 
3.16. Interface profile evolution for the case of conductivity ratio of 100 , density ratio of 0.752 , permittivity ratio of 10.974 , viscosity ratio of 0.284 with an initial sausage-mode perturbation of $k^{*}=10.0$ and double the grid resolution compared to the previous cases. Conductivity and charge density snapshots at $80 \mu s$ are given in (a) and (b) respectively. Snapshots at $120 \mu \mathrm{s}$ are provided in (c) and

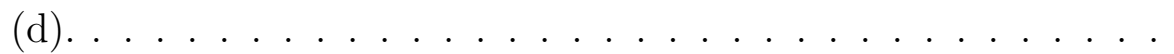




\section{Chapter 1}

\section{Introduction}

\subsection{Electrohydrodynamics in microchannel flows}

Microfluidic systems usually utilize microchannels of planar geometry which are typically 10-100 $\mu \mathrm{m}$ wide, 1-50 $\mu \mathrm{m}$ deep and several mm long. The key components of a microfluidic control system include micro-pumps, micro-valves, microactuators and micro-sensors. In the last fifteen years, there has been a growing demand for analyzing biological materials such as proteins, DNA, cells and chemical reagents using microfluidic devices. The advantages of these devices include substantially reduced sample size, extremely short analysis times, high throughput and portability. Towards achieving this goal, development of miniaturized diagnostic platforms called micro total analysis systems $(\mu T A S)$ which mimic conventional laboratory processes on microchips have been attracting extensive research studies over the past decade. In particular, active and passive micromixing techniques have received increased attention with developments in microreactors, where two chemical species are mixed prior to introducing them into a reaction chamber. Micromixers are concerned with efficient mixing of two or more miscible or immiscible phases and being severely disadvantaged by the 
low Reynolds number regimes in a typical microchannel flow, employ either a complex geometry modification (passive mixing) or an external field (active mixing) to improve mixing. A basic understanding and modeling of microfluidic processes are essential for optimizing the performance of these microdevices.

Electrokinetics is the study of electric field and flow field interfaction close (usually a few nanometers) to the microchannel walls. Solid-liquid interfaces in the presence of electrolytes (aqueous buffer solutions) carry electrostatic charge, as a result of which there is an electric double layer (EDL) in the region close to the channel wall [1]. The EDL field is fundamentally responsible for two basic electrokinetic phenomena: electroosmosis and electrophoresis. Electroosmosis is related to the fluid flow due to interaction between the EDL close to the walls and the applied electric field while electrophoresis is the motion of a charged particle relative to the surrounding fluid under an applied electric field. On the contrary, electrohydrodynamics (EHD) is the study of electric field effects on the fluid flow in the bulk (far away from the walls). Electrohydrodynamic theory has been used to explain the evolution of liquid-liquid interfaces in the bulk, far from the solidliquid interfaces, where electrokinetic effects are negligible. The interface between two miscible/immiscible fluids with different electrical properties (like electrical conductivity) can be destabilized with an applied electric field due to charge deposition on the interface and henceforth, an electric body force acting on the interface. Such an instability is termed electrohydrodynamic (EHD) instability and has been actively studied since Melcher's initial works [17], [18] on the subject. 


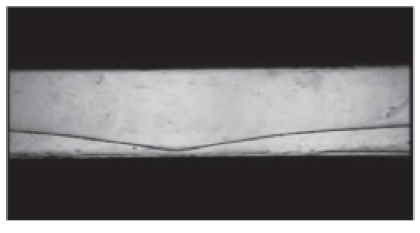

(a) Deformation.

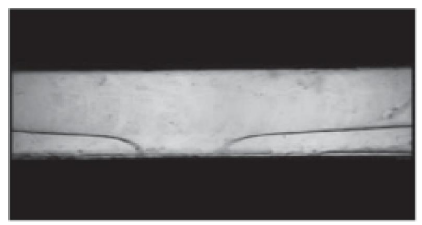

(b) Rupture.

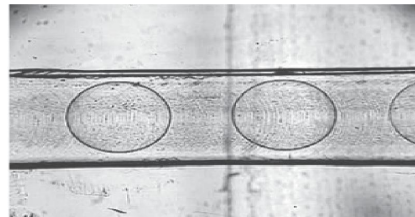

(c) Droplet formation.

Figure 1.1: EHD instability experiments in a two fluid interface [15]. Interfacial deformation, rupture and subsequent droplet formation can be observed.

The use of EHD instabilities to induce mixing in miscible fluids has been reported in the experimental work of Oddy et al. [10], Glasgow et al. [11] and Lin et al. [12]. They have also been used in microchannels for the generation of droplets in immiscible fluids and have been experimentally observed using a two-fluid interface by Ozen et al. [15] as seen in figure 1.1. Another potential application explored by Zahn and Reddy [3] is aimed at utilizing EHD instabilities towards miniaturizing the organic-aqueous extraction technique for DNA purification.

\subsection{Motivation: Organic-aqueous extraction}

Genomic or plasmid DNA extraction using aqueous-organic liquids is one of the most commonly used techniques for DNA purification in biological laboratories (Sambrook et al. [2]). Briefly speaking, cells are lysed in an aqueous solution

(the lysis buffer), and then mixed with an equal volume of organic fluid (phenol, chloroform and isoamyl alcohol, 25:24:1 by volume). Cell components naturally distribute themselves, termed "biological partitioning", into the two immiscible phases: the membrane components and proteins partition to the organic-aqueous 


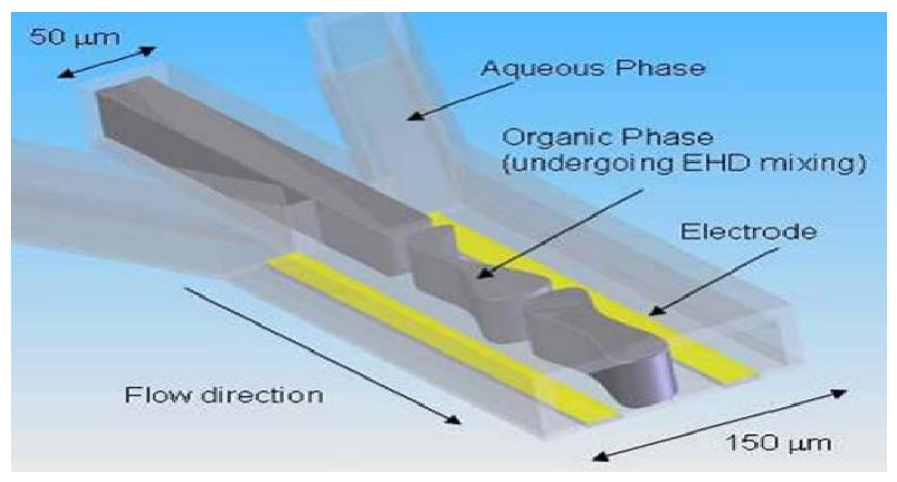

Figure 1.2: Schematic of the set-up for EHD instability in a three-layer stratified flow [3]. The electric field is applied perpendicular to the direction of flow.

interface, whereas DNA stays in the aqueous phase. The latter phase containing the purified DNA is then extracted for further processing. In order to efficiently partition the cell components, the two phases need to be well-dispersed to maximize the surface areas in contact and maximize DNA purification. This "mixing" step is particularly challenging when implementing the technique in a microfluidic platform. In a previous work by Zahn and Reddy [3], it was demonstrated that EHD instability could be utilized to break a stream into droplets and increase the interfacial area available for partitioning. A schematic of the experimental set-up of Zahn and Reddy [3] is shown in figure 1.2. We can observe a threeinlet geometry in which an organic solution is sandwiched between two layers of aqueous solutions and an electric field is applied perpendicular to the direction of flow. This induces electrohydrodynamic instability leading to interfacial deformation and droplet formation. Some of the experimental observations are shown in figure 1.3. 


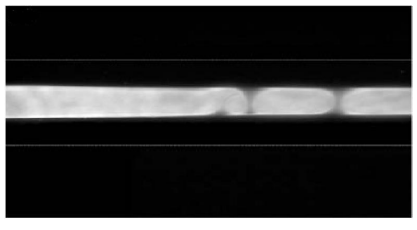

(a) $E_{0}=8.2 \times 10^{5} \mathrm{~V} / \mathrm{m}$.

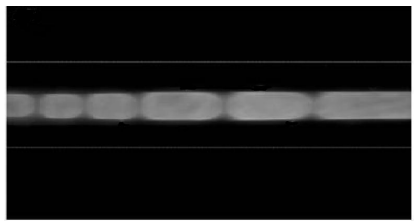

(b) $E_{0}=8.4 \times 10^{5} \mathrm{~V} / \mathrm{m}$.

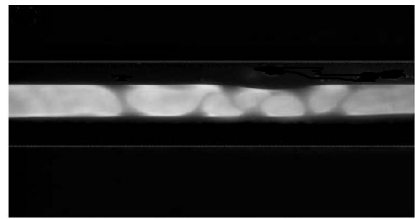

(c) $E_{0}=8.8 \times 10^{5} \mathrm{~V} / \mathrm{m}$.

Figure 1.3: EHD instability experiments in a three-layer stratified flow [3]. The formation of droplets and enhanced mixing as electric field is increased can be observed.

\subsection{Previous work}

Yih [4] first analyzed the problem of hydrodynamic instability due to viscosity stratification in a two-layer fluid flow for both plane Couette and plane Poiseuille base flows. Using a method of non-singular perturbation for long waves ([5]), he observed the presence of an unstable mode for a finite Reynolds number flow, however small the Reynolds number was, and concluded that this unstable mode was primarily due to the viscosity stratification. This mode is referred to as an "interfacial mode". Following Yih's work, Li [6] extended the stability analysis to plane Couette base flow with three-layer fluids of different viscosities and noted the existence of two modes of disturbances corresponding to the two surfaces of discontinuity. Refering to Yih's conclusion of the cause of instability due to viscosity stratification, Li also pointed out that the flow can be stabilizing or destabilizing depending on the viscosity ratio and depth ratio of the fluids. Yiantsios and Higgins [9], utilizing Hooper and Boyd's [7] asymptotic analysis for Couette flow in an infinite region, studied the linear stability of plane Poiseuille 
flow of two superimposed fluids of different densities and viscosities. They essentially extended Yih's work for small wavenumbers [4] to large wavenumbers and also considered the effects of density ratio, thickness ratio, gravity and interfacial tension. Explaining the instabilities not only due to the "interfacial mode" (as observed by Yih) but also to "shear mode" of the Tollmien-Schlichting type for high Reynolds number, they asymptotically analyzed the long-wave and shortwave cases to compare with the experimental results of Kao and Park [8]. Joseph and Renardy [22] provide an extensive summary of linear analysis of two-fluid flows.

For analyzing EHD instability, two different approaches have been considered: (1) surface coupled model, in which the charges are assumed to accumulate only on the interface and hence, the electric stresses are coupled to the fluid flow only at the interface and (2) bulk coupled model, in which charges accumulate over a diffuse interface with a conductivity gradient along its thickness and the diffuse interface experiences an electric body force. Melcher and co-workers first used the surface coupled model to study stability of a two fluid interface subject to a parallel electric field [17] and perpendicular electric field [18]. Hoburg and Melcher [19] later studied the two fluid diffuse interfacial stability subject to a parallel electric field using bulk coupled model and included viscous diffusion effects. Mohamed et al. [20] examined the electrohydrodynamic stability of an interface, formed by an upper conducting fluid and a lower dielectric fluid of different viscosities and densities, which is subjected to a normal electric field 
between two parallel plates. A long-wave asymptotic analysis was performed for a plane Couette-Poiseuille base flow. They concluded that the normal electric field produced destabilizing effects for all Reynolds numbers and the stability was enhanced if the depth of the lower layer was increased. Abdella and Rasmussen [21] concentrated on linear stability of a planar interface between two unbounded fluids with different viscosities, densities and electrical properties subjected to a Couette flow and derived the dispersion relation in terms of Airy functions and integrals. They considered two limiting cases: one in which the fluid interface is perfectly conducting and hence supports free charges and the other in which the interface does not support any free charges as a result of which polarization charges play a key role in the instability and electrical shear forces are zero at the interface. G.I. Taylor introduced the concept of leaky dielectrics in his work [16] in which he mentioned that immiscible fluids when subjected to electric fields resulted in the accumulation of electric charges on their interfaces irrespective of the magnitude of electrical conductivities of either fluids. Melcher and his coworkers [17], [18], [19] used the theory extensively to study electrohydrodynamic flows and the theory has been summarized along with potential applications by Saville [26].

Several experimental and numerical works have been reported for EHD instability in microchannels. Lin et al. [12], following Melcher's framework for a bulk-coupled model and considering diffusion of electrical conductivity as in Baygents and Baldessari [13], numerically analyzed the electrokinetic temporal 
instability of a diffuse inteface formed between two miscible fluids with a conductivity gradient. Based on their linear analysis for 2D, 3D cases and fully nonlinear simulations using spectral methods, they emphasized the increased stability due to the presence of side walls and detailed the range of validity of the electroviscous scaling proposed by Hoburg and Melcher [19]. Chen et al. [14] used depth-averaged linearized governing equations to study the convective and absolute instabilities in the microchannel. Ozen et al. [23], following their experimental work [15], derived dispersion relations for the case of an interface between two immiscible leaky dielectrics in a plane Couette Poiseuille base flow and solved the eigenvalue problem using Chebyshev pseudospectral methods. For the case when the charge relaxation times are much smaller than the fluid flow time scale, they concluded that the permittivity and conductivity ratios play an important role in determining if the electric field plays a stabilizing or a destabilizing role. Li et al. [24] performed a long-wave asymptotic analysis for the same problem, compared the results with the numerical solution obtained using Chebyshev pseudospectral methods for all wavenumbers and highlighted the importance of the electrical property ratios and surface tension effects. Furthermore, Uguz et al. [25] provided a qualitative comparison of the linear stability of the interface between two leaky dielectrics for the cases of parallel and perpendicular electric fields, concluding that the normal electric field had a destabilizing effect over a wider range of parametric values compared to that of the parallel electric field. Zahn et al. [3] theoretically derived the dispersion relations for a three-layer stratified 
flow configuration with a zero base flow and a limiting case of infinite conductivity ratio between the sandwiching and sandwiched fluid. The most interesting characteristics observed are two distinct modes called the "kink" and "sausage" modes, with the kink mode having the two interfacial perturbations in phase with each other and the sausage mode having the two interfacial perturbations out of phase by $\pi$. Our current work focuses on the linear stability analysis for a generalized three-layer EHD flow with property variations and a Plane-Poiseuille base flow.

Apart from the linear stability analysis of EHD flows, understanding the breakup of an interface separating immiscible fluids of different mechanical and electrical properties is of considerable importance in microfluidic applications like optimizing aqueous-organic extraction. Significant progress has been made recently with regard to numerical simulations of multiphase flow, especially homogeneous bubbly flows and drops in channel flows. Numerical simulations of electrohydrodynamic flows of droplets considering simplified flow assumptions like Stokes flow or inviscid flow have been conducted by several authors [27], [28] using boundary integral methods. The axisymmetric interaction of drops in the limiting case of zero Reynolds number was comprehensively studied by Baygents et al. [29] using boundary integral technique. Basaran et al. [30]-[33] utilized finite element methods to simulate drop deformations of pendant and sessile conducting drops due to electric field and characterized equilibrium drop shapes as well as their morphological evolution. Tsukuda et al. [34], using Galerkin finite 
element method, described the circulation produced inside and outside a single suspended drop and subsequently, studied the effects of gravity and electrohydrodynamic forces [35]. The results were found to be in good agreement with their experimental observations and Taylor's theoretical results. Fernandez et al. [36] studied the response of an emulsion of drops in a channel subjected to an electric field using interface front tracking method [37] and concluded that the drop distribution depends strongly on the dielectric properties of both fluids. Zhang and Kwok [46] simulated 2D drop deformation in electric field using a Lattice Boltzmann method. A few numerical simulations for behaviour of flat interfaces with and without the influence of an electric field have also been reported. Tauber et al. [45] analyzed the Kelvin-Helmholtz instability of an initial flat interface between two immiscible fluids using front-tracking method and explained the formation of 2D fingers for different density ratios, Reynolds numbers and Weber numbers. Recently, Tomar et al. [47] devised a numerical method to simulate two-phase electrohydrodynamic flows using a combination of volume of fluid (VOF) and level set (LS) methods and presented results for the cases of flat interface between two dielectric fluids and droplets. In our work, we extend the interface front tracking method [37] for simulating interface evolution in a three-layer stratified flow in the presence of an electric field directed parallel to the conductivity gradient. 


\subsection{Issues and challenges}

Electrohydrodynamic mixing has been a subject of investigation in the past decade. In the case of immiscible fluids, the mixing involves a complex coupling between the fluid flow (viscous stresses) and electric surface forces (Maxwell stresses) due to charge accumulation at the interfaces. Such a complex multiscale and multiphysics process makes it difficult for a theoretical analysis without various simplifying assumptions. Currently, the formation of droplets resulting from interfacial rupture is a subject of intense research and as on date, a cogent model to explain the physics of this phenomenon is still in progress. Numerical models using level set or volume of fluid methods use an arbitrary scheme based on gridsize to artificially rupture the interfaces to form droplets. As mentioned above, though several computational models have been developed to address this process, a complete three-dimensional (3D) computational model which employs interface rupture and subsequent droplet formation is further needed. Such a tool would provide a detailed understanding of this complex, multiscale and multiphysics process.

\subsection{Scope of the thesis}

In this work, analysis and simulation are combined to study two phase electrohydrodynamic instability. In the problem configuration, the organic (phenol) phase flows into the microchannel in parallel with and sandwiched between two aqueous streams, creating a three layer planar geometry, the two liquid phases are 
immiscible. An electric field is applied to induce instability and break the organic stream into droplets. We consider a two-dimensional (2D) three-layer stratified, immiscible fluid flow with non-uniformities in density, viscosity, dielectric permittivity and electrical conductivity. The objective of this thesis is two-fold - (a) To perform linear stability analysis using Chebyshev pseudo-spectral methods and (b) a 2D nonlinear numerical simulation using a finite volume/front tracking immersed boundary technique. The combination of the linear analysis and nonlinear simulation provides us the necessary tools to investigate and optimize droplet dispersion in favor of efficient DNA extraction. The linear stability analysis is presented in Chapter 2. The numerical methodology for the fully nonlinear simulation is presented in Chapter 3. The discussion on results from linear analysis and nonlinear simulations are provided in Chapters 2 and 3 respectively. The main conclusions and future work are also outlined in Chapter 4 . 


\section{Chapter 2}

\section{Linear stability analysis}

\subsection{Problem specification}

We consider two-dimensional, three-layer viscous stratified flow of immiscible fluids in a microchannel subjected to a constant applied electric field $E_{0}$ in the positive y-direction (figure 2.1). The channel is bounded by two infinitely long flat plates placed parallel to the $X$-axis in the $X Y$ coordinate system as shown. The height of the channel is $2 a+b$, where $a$ and $b$ are the thickness of fluid layers "a" and "b" respectively. The sandwiching fluid (layers 1 and 3) has properties $\rho_{1}, \mu_{1}, \epsilon_{1}$ and $\sigma_{1}$ while the sandwiched fluid (layer 2) has different properties $\rho_{2}, \mu_{2}, \epsilon_{2}$ and $\sigma_{2}$. Here, $\rho, \mu, \epsilon$ and $\sigma$ refer to the density, viscosity, dielectric permittitivity and electrical conductivity of the fluids respectively. Furthermore, we assume the immiscible fluids under consideration to be incompressible, leaky dielectrics and employ the "surface-coupled" model to study the interfacial deformation characteristics (as against the "bulk-coupled" model considered by Lin et al. [12]). We also assume that the gravity forces have negligible effects. The interfacial deformations of the top and bottom interfaces are given by $\zeta_{1}$ and $\zeta_{2}$. The interface normals are then obtained as $\mathbf{n}_{1}=\left[-\zeta_{1 x}, 1\right]$ and $\mathbf{n}_{2}=\left[-\zeta_{2 x}, 1\right]$ 


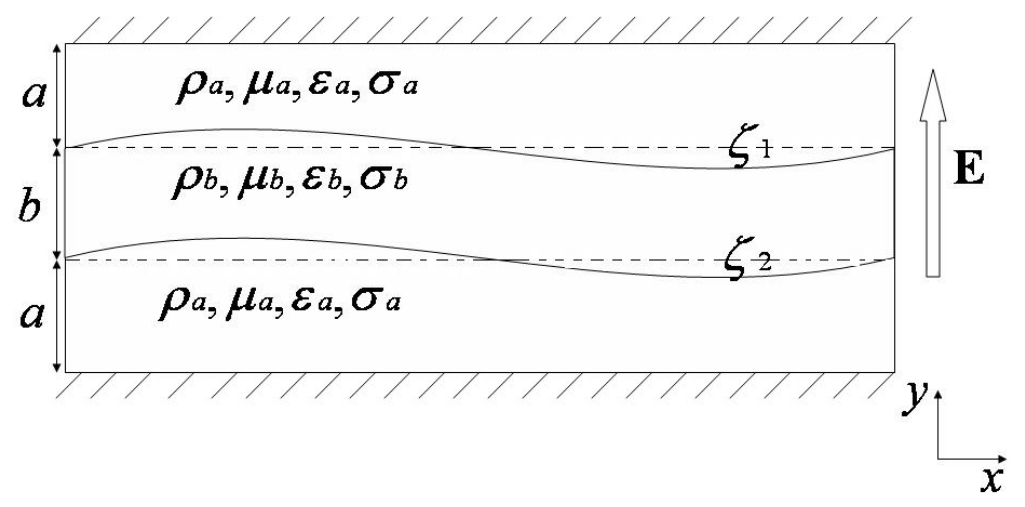

Figure 2.1: Two dimensional domain for linear analysis of three-layer viscous stratified flow. The schematic shows the electric field applied parallel to the conductivity gradient.

respectively, where the subscript " $x$ " denotes the partial differentiation with respect to $x$. Correspondingly, the tangent vectors at the two interfaces become $\mathbf{t}_{1}=\left[1, \zeta_{1 x}\right]$ and $\mathbf{t}_{2}=\left[1, \zeta_{2 x}\right]$ respectively. A convective base flow (Plane-Poiseuille flow) may also be imposed.

\subsection{Governing equations}

The fluid motion is governed by the continuity and Navier-Stokes equations in each layer $(j=1,2$ and 3$)$ as:

$$
\begin{gathered}
\nabla \cdot \mathbf{u}^{(j)}=0 \\
\rho_{j}\left(\frac{\partial \mathbf{u}^{(j)}}{\partial t}+\mathbf{u}^{(j)} \cdot \nabla \mathbf{u}^{(j)}\right)=-\nabla p+\mu_{j} \nabla^{2} \mathbf{u}^{(j)},
\end{gathered}
$$

No-slip boundary conditions for velocities are specified at the wall. The absence of an electric body force term on the right-hand side of the Navier-Stokes equation 
is a consequence of the surface-coupled model, which stipulates (1) a jump in electrical properties (electrical conductivity and dielectric permittivity) at the interfaces and (2) zero electric charge density and hence, zero electric body forces in the bulk. The electric field in each layer is related to the electric potential as $\mathbf{E}^{(j)}=-\nabla \Phi^{(j)}$. In absence of charge density in the bulk, the charge density evolution equation reduces to the Ohmic equation [12].

$$
\nabla \cdot\left(\sigma \nabla \Phi^{(j)}\right)=0
$$

Since the electrical conductivities are constant in each layer, the Ohmic equation reduces to Laplace's equation for electric potential distribution in each layer:

$$
\nabla^{2} \Phi^{(j)}=0
$$

with a constant potential of $V_{0}$ at the lower wall and zero potential at the upper wall being specified. The total stress tensor $\tau$ is the sum of (1) the fluid stress tensor $T^{f}$, which includes pressure and viscous stress tensor, and (2) the Maxwell's electric stress tensor $T^{e}$ :

$$
\begin{aligned}
\tau & =T^{f}+T^{e} \\
T^{f} & =-p I+\mu\left(\nabla \mathbf{u}+\nabla \mathbf{u}^{T}\right), \\
T^{e} & =\epsilon \mathbf{E} \mathbf{E}-\frac{1}{2} \epsilon|\mathbf{E}|^{2} I,
\end{aligned}
$$


The problem is completely specified with the jump conditions on each interface. We denote the jump discontinuity of a variable "." across the interface as $\|$.$\| .$

Pertaining to the electrical variables on each interface, we require the continuity of electric potential and continuity of Ohmic current:

$$
\begin{gathered}
\|\Phi\|=0, \\
\|\sigma \nabla \Phi \cdot \mathbf{n}\|=0,
\end{gathered}
$$

Regarding the flow variables, the velocities are continuous across each interface and the interfaces advect with the local fluid velocities. In an equational form, these conditions can be expressed as:

$$
\begin{gathered}
\|\mathbf{u}\|=0, \\
v=\frac{D \zeta}{D t},
\end{gathered}
$$

where, $D$ denotes the material derivative (following the particle). While the total tangential stress is continuous across each interface, the jump in the total normal stress is balanced by the surface tension forces. We denote the surface tension coefficient by $\gamma$ and the interfacial curvature by $\kappa$. The jump in the total tangential and normal stresses can be written as:

$$
\|\mathbf{t} \cdot \tau \cdot \mathbf{n}\|=0
$$




$$
\|\mathbf{n} \cdot \tau \cdot \mathbf{n}\|+\gamma \kappa=0
$$

Equations (2.2.1)-(2.2.10) along with the relevant boundary conditions at the walls need to be solved to obtain the velocity field.

\subsection{Numerical solution via pseudospectral methods}

Before discussing the numerical method to study the stability of the system at arbitrary wavenumbers, we first derive the governing equations for the long-wave asymptotic behaviour for small wavenumbers or long wavelengths $(k \rightarrow 0)$. The detailed derivation considering a convective base flow is given in Appendix A. The primary intention is to enumerate the governing equations, although an analytical solution exists for the final forms of equations derived herein (using a procedure similar to the one outlined in [24]). The discussion of the analytical solution would be taken up in future works. Since long-wave asymptotic methods are limited to very small wavenumbers, numerical methods are required for analyzing the system behaviour at arbitrary wavenumbers. Spectral methods have been found particularly to be attractive because of ease of implementation and high accuracy. Chebyshev pseudospectral method has been employed in our current work, which eventually reduces the perturbation analysis to an "eigenvalue problem" (explained in later sections). On the other hand, the perturbation analysis in Zahn and Reddy [3], whose earlier work for infinite electrical conductivity ratio is currently extended to general electrical conductivity ratios, results in a "root-finding problem". It is worth pointing out that though the details of the 
"root-finding" solution would not be provided in this report, the results from the pseudospectral method provided in this report were verified against the results from the root-finding method and both are in excellent agreement with each other. Most importantly, the "root-finding" methodology is applicable only for the case of zero convective base flow.

\subsubsection{Scaling and dimensionless parameters}

The governing equations are made dimensionless by using $d=b / 2$ as the characteristic length scale, the electro-viscous velocity $U_{e v}=\epsilon_{2} E_{0}^{2} d / \mu_{2}$ as the velocity scale and $t^{*}=d / U_{e v}$ as the time scale. The pressure is scaled by $p^{*}=\mu_{2} U_{e v} / d$. The electric potential and electric field are scaled with $V_{0}$ and $E_{0}$ respectively. The fluid properties (electrical and mechanical) are scaled with respect to the properties of the sandwiched fluid. The major dimensionless parameters are:

$$
\begin{aligned}
\eta & =2 n+1, n=\frac{a}{b} \\
\sigma & =\frac{\sigma_{1}}{\sigma_{2}}, \epsilon=\frac{\epsilon_{1}}{\epsilon_{2}}, \mu=\frac{\mu_{1}}{\mu_{2}}, \rho=\frac{\rho_{1}}{\rho_{2}}, \\
R e_{1,2} & =\frac{\rho_{1,2} U_{e v} d}{\mu_{2}}, \\
C a & =\frac{\mu U_{e v}}{\gamma}
\end{aligned}
$$

Here, Re is the Reynolds number and $\mathrm{Ca}$ is the capillary number, which is the ratio of viscous force to the interfacial tension force. 


\subsubsection{Dimensionless governing equations}

Based on the scaling considered, the Navier stokes equation in each layer is given as

$$
\begin{aligned}
& R e_{1}\left(\frac{\partial \mathbf{u}_{1}}{\partial t}+\mathbf{u}_{1} \cdot \nabla \mathbf{u}_{1}\right)=-\nabla p_{1}+\mu \nabla^{2} \mathbf{u}_{1},-\mathrm{h} \leq \mathrm{y}_{1} \leq \mathrm{h} \\
& R e_{2}\left(\frac{\partial \mathbf{u}_{2}}{\partial t}+\mathbf{u}_{2} \cdot \nabla \mathbf{u}_{2}\right)=-\nabla p_{2}+\mu \nabla^{2} \mathbf{u}_{2},-1 \leq \mathrm{y} \leq 1 \\
& R e_{1}\left(\frac{\partial \mathbf{u}_{3}}{\partial t}+\mathbf{u}_{3} \cdot \nabla \mathbf{u}_{3}\right)=-\nabla p_{3}+\mu \nabla^{2} \mathbf{u}_{3},-\mathrm{h} \leq \mathrm{y}_{1} \leq \mathrm{h}
\end{aligned}
$$

The forms of other governing equations, boundary and jump conditions remain the same as in (2.2.1)-(2.2.9) except for the normal stress jump condition (2.2.10). In dimensionless terms, the jump in the normal stress is expressed as:

$$
\|\mathbf{n} \cdot \tau \cdot \mathbf{n}\|+\frac{1}{C a} \kappa=0
$$

\subsubsection{Perturbation analysis}

We are primarily interested in perturbation analysis of a base state with a Poiseuille flow of zero velocity. Following the standard perturbation analysis procedure, we denote the perturbation variables with a prime $\left(^{\prime}\right)$ and the base state variables with a subscript of "0". Hence, the total velocity, pressure, electric potential and electric field are denoted as $\mathbf{u}=\left[U+u^{\prime}, v^{\prime}\right], p=p_{0}+p^{\prime}, \Phi=\Phi_{0}+\Phi^{\prime}$ and $\mathbf{E}=\left[E_{x}^{\prime}, E_{0}+E_{y}^{\prime}\right]$ respectively (The subscripts in the perturbation components of electric field imply $\mathrm{x}-$, $\mathrm{y}$ - components and not partial differentiation). The 
generalized base state Poiseuille flow velocity profile can be obtained as:

$$
\begin{aligned}
U_{1} & =\frac{U_{c}}{\mu}\left(y_{1}^{2}+2(1+h) y_{1}-3 h^{2}-2 h\right),-\mathrm{h} \leq \mathrm{y}_{1} \leq \mathrm{h} \\
U_{2} & =U_{c}\left(\left(y_{1}^{2}-1\right)-\frac{4}{\mu}\left(h^{2}+h\right)\right),-1 \leq \mathrm{y} \leq 1 \\
U_{3} & =\frac{U_{c}}{\mu}\left(y_{1}^{2}-2(1+h) y_{1}-3 h^{2}-2 h\right),-\mathrm{h} \leq \mathrm{y}_{1} \leq \mathrm{h} \\
U_{c} & =\frac{1}{2} \frac{\partial p}{\partial x},
\end{aligned}
$$

where, $U_{c}$ is a user-defined parameter (known). Also, the base state electric field in each layer can be obtained as:

$$
\begin{aligned}
& E_{1}=E_{0} \frac{2 a+b}{2 a+\sigma b}=E_{0} \frac{\eta}{\eta+\sigma-1}, \\
& E_{2}=E_{0} \sigma \frac{2 a+b}{2 a+\sigma b}=E_{0} \sigma \frac{\eta}{\eta+\sigma-1}, \\
& E_{3}=E_{0} \frac{2 a+b}{2 a+\sigma b}=E_{0} \frac{\eta}{\eta+\sigma-1},
\end{aligned}
$$

The continuity equation for perturbation flow velocities (dimensionless) in each layer is given by:

$$
\begin{aligned}
& \frac{\partial u_{1}^{\prime}}{\partial x}+\frac{\partial v_{1}^{\prime}}{\partial y}=0, \\
& \frac{\partial u_{2}^{\prime}}{\partial x}+\frac{\partial v_{2}^{\prime}}{\partial y}=0 \\
& \frac{\partial u_{3}^{\prime}}{\partial x}+\frac{\partial v_{3}^{\prime}}{\partial y}=0
\end{aligned}
$$

On substituting the flow variables (base + perturbation) into the dimensionless Navier Stokes' equation (2.3.5a), we have for $\mathrm{x}$-momentum and $\mathrm{y}$-momentum 
equations for fluid layer 1:

$$
\begin{aligned}
& R e_{1}\left(\frac{\partial u_{1}^{\prime}}{\partial t}+u_{1}^{\prime} \frac{\partial u_{1}^{\prime}}{\partial x}+v_{1}^{\prime} \frac{\partial u_{1}^{\prime}}{\partial y}\right)=-\frac{\partial p_{1}^{\prime}}{\partial x}+\frac{\partial^{2} u_{1}^{\prime}}{\partial x^{2}}+\frac{\partial^{2} u_{1}^{\prime}}{\partial y^{2}} \\
& R e_{1}\left(\frac{\partial v_{1}^{\prime}}{\partial t}+u_{1}^{\prime} \frac{\partial v_{1}^{\prime}}{\partial x}+v_{1}^{\prime} \frac{\partial v_{1}^{\prime}}{\partial y}\right)=-\frac{\partial p_{1}^{\prime}}{\partial y}+\frac{\partial^{2} v_{1}^{\prime}}{\partial x^{2}}+\frac{\partial^{2} v_{1}^{\prime}}{\partial y^{2}}
\end{aligned}
$$

Similarly, from (2.3.5b) for layer 2:

$$
\begin{aligned}
& R e_{2}\left(\frac{\partial u_{2}^{\prime}}{\partial t}+u_{2}^{\prime} \frac{\partial u_{2}^{\prime}}{\partial x}+v_{2}^{\prime} \frac{\partial u_{2}^{\prime}}{\partial y}\right)=-\frac{\partial p_{2}^{\prime}}{\partial x}+\frac{\partial^{2} u_{2}^{\prime}}{\partial x^{2}}+\frac{\partial^{2} u_{2}^{\prime}}{\partial y^{2}} \\
& R e_{2}\left(\frac{\partial v_{2}^{\prime}}{\partial t}+u_{2}^{\prime} \frac{\partial v_{2}^{\prime}}{\partial x}+v_{2}^{\prime} \frac{\partial v_{2}^{\prime}}{\partial y}\right)=-\frac{\partial p_{2}^{\prime}}{\partial y}+\frac{\partial^{2} v_{2}^{\prime}}{\partial x^{2}}+\frac{\partial^{2} v_{2}^{\prime}}{\partial y^{2}}
\end{aligned}
$$

and likewise, from $(2.3 .5 \mathrm{c})$ for layer 3 :

$$
\begin{aligned}
R e_{1}\left(\frac{\partial u_{3}^{\prime}}{\partial t}+u_{3}^{\prime} \frac{\partial u_{3}^{\prime}}{\partial x}+v_{3}^{\prime} \frac{\partial u_{3}^{\prime}}{\partial y}\right) & =-\frac{\partial p_{3}^{\prime}}{\partial x}+\frac{\partial^{2} u_{3}^{\prime}}{\partial x^{2}}+\frac{\partial^{2} u_{3}^{\prime}}{\partial y^{2}} \\
R e_{1}\left(\frac{\partial v_{3}^{\prime}}{\partial t}+u_{3}^{\prime} \frac{\partial v_{3}^{\prime}}{\partial x}+v_{3}^{\prime} \frac{\partial v_{3}^{\prime}}{\partial y}\right) & =-\frac{\partial p_{3}^{\prime}}{\partial y}+\frac{\partial^{2} v_{3}^{\prime}}{\partial x^{2}}+\frac{\partial^{2} v_{3}^{\prime}}{\partial y^{2}}
\end{aligned}
$$

The no-slip conditions on the upper and lower wall result in: $u_{1}^{\prime}=v_{1}^{\prime}=u_{3}^{\prime}=$ $v_{3}^{\prime}=0$. The electric potential perturbations obey the Laplace's equation in each layer, since the base state electric potentials obey the same:

$$
\begin{aligned}
& \frac{\partial^{2} \Phi_{1}^{\prime}}{\partial x^{2}}+\frac{\partial^{2} \Phi_{1}^{\prime}}{\partial y^{2}}=0 \\
& \frac{\partial^{2} \Phi_{2}^{\prime}}{\partial x^{2}}+\frac{\partial^{2} \Phi_{2}^{\prime}}{\partial y^{2}}=0
\end{aligned}
$$




$$
\frac{\partial^{2} \Phi_{3}^{\prime}}{\partial x^{2}}+\frac{\partial^{2} \Phi_{3}^{\prime}}{\partial y^{2}}=0
$$

The constant potentials applied at the upper and lower wall lead to: $\Phi_{1}^{\prime}=\Phi_{3}^{\prime}=$ 0. Expanding the potential continuity and Ohmic current equations (2.2.5) and (2.2.6), and neglecting terms second-order and higher, we get the dimensionless forms as:

$$
\begin{gathered}
\left\|-E \frac{\partial \zeta}{\partial x}+\frac{\partial \Phi^{\prime}}{\partial x}\right\|=0, \\
\left\|\sigma\left(-E+\frac{\partial \Phi^{\prime}}{\partial y}\right)\right\|=0,
\end{gathered}
$$

where, $\sigma$ is the conductivity ratio and $\mathrm{E}$ is the base state electric field in the layer. Similarly, substituting the field variables into the stress continuity equations (2.2.9) and (2.2.10), expanding and neglecting terms second-order and higher, the dimensionless forms are obtained as:

$$
\begin{gathered}
\|\mathbf{t} \cdot \tau \cdot \mathbf{n}\|=\left\|\mu\left(\frac{\partial u^{\prime}}{\partial y}+\frac{\partial v^{\prime}}{\partial x}\right)+\epsilon E^{2} \zeta_{x}+\epsilon E E_{x}^{\prime}\right\|=0 \\
\|\mathbf{n} \cdot \tau \cdot \mathbf{n}\|=\left\|-p^{\prime}+2 \mu \frac{\partial v^{\prime}}{\partial y}+\epsilon E E_{y}^{\prime}\right\|+\frac{1}{C a} \frac{\partial^{2} \zeta}{\partial x}=0,
\end{gathered}
$$

where, $\mu$ is the viscosity ratio in the layer and $\epsilon$ is the dielectric permittivity ratio. Equations (2.3.7)-(2.3.17) represent the linearized equations satisfied by the perturbation variables and together with the boundary conditions, can be solved to determine the perturbation variables and their evolution.

In our current work, we are primarily concerned with the temporal stability analysis and hence, we assume perturbations of the form $f^{\prime}=\hat{f}(y) e^{i k x+s t}$, where 
" $f$ " represents any perturbation variable (velocity, electric potential etc.). Here, " $k$ " is the real wavenumber and " $s "$ is the complex growth rate i.e. $s=s_{r}+i s_{i}$ where $s_{r}$ is the real part and $s_{i}$ is the imaginary part of the growth rate. From the definition, we can state that the system would be "stable" if $s_{r}$ is negative while the system would be "unstable" if $s_{r}$ is positive. Convective stability analysis of this system would be considered in our future work. Proceeding with the temporal analysis and considering fluid layer $1\left(-\mathrm{h} \leq \mathrm{y}_{1} \leq \mathrm{h}\right)$, the $\mathrm{x}$-momentum equation of (2.3.10a) is expressed as:

$$
R e_{1}\left(s \hat{u}_{1}\right)=-i k \hat{p}_{1}+\mu\left(D^{2}-k^{2}\right) \hat{u}_{1}
$$

subject to the no-slip condition at the wall and velocity continuity (2.2.7):

$$
\hat{u}_{1}(h)=0, \hat{u}_{1}(-h)+D U_{1}(-h) \hat{\zeta}_{1}=\hat{u}_{2}(1)+D U_{2}(1) \hat{\zeta}_{1},
$$

and the $\mathrm{y}$-momentum equation of $(2.3 .10 \mathrm{~b})$ can be written as:

$$
\operatorname{Re}_{1}\left(s \hat{v}_{1}\right)=-D \hat{p}_{1}+\mu\left(D^{2}-k^{2}\right) \hat{v}_{1}
$$

subject to the no-slip condition at the wall and velocity continuity (2.2.7):

$$
\hat{v}_{1}(h)=0, \hat{v}_{1}(-h)=\hat{v}_{2}(1),
$$


and finally, the continuity equation (2.3.9a) is given by:

$$
i k \hat{u}_{1}+D \hat{v}_{1}=0
$$

Here, "D" in the above equations denotes differentiation with respect to "y". Proceeding similarly for layer 2, the x-momentum (2.3.11a), y-momentum (2.3.11b) and continuity (2.3.9b) equations are expressed as:

$$
\begin{gathered}
\operatorname{Re}_{2}\left(s \hat{u}_{2}\right)=-i k \hat{p}_{2}+\mu\left(D^{2}-k^{2}\right) \hat{u}_{2}, \\
\operatorname{Re}_{2}\left(s \hat{v}_{2}\right)=-D \hat{p}_{2}+\mu\left(D^{2}-k^{2}\right) \hat{v}_{2}, \\
i k \hat{u}_{2}+D \hat{v}_{2}=0,
\end{gathered}
$$

The perturbation electric potential Laplace equation for layer 1 is written as:

$$
\left(D^{2}-k^{2}\right) \hat{\phi}_{1}=0
$$

subject to the zero perturbation at the upper wall and the potential continuity condition from (2.3.14):

$$
\hat{\phi}_{1}(h)=0,-E_{1} \hat{\zeta}_{1}+\hat{\phi}_{1}(-h)=-E_{2} \hat{\zeta}_{1}+\hat{\phi}_{2}(1)
$$

From the normal and tangential stress matching conditions (2.3.16) and (2.3.17) 
for the top interface, we get:

$$
\begin{gathered}
-\left(\mu\left(D \hat{u}_{1}(-h)+i k \hat{v}_{1}(-h)\right)+i k \epsilon E_{1}^{2} \hat{\zeta}_{1}-i k \epsilon E_{1} \hat{\phi}_{1}(-h)\right) \\
+\left(D \hat{u}_{2}(1)+i k \hat{v}_{2}(1)\right)+i k \epsilon E_{2}^{2} \hat{\zeta}_{1}-i k \epsilon E_{2} \hat{\phi}_{2}(1)=0 \\
-\left(-\hat{p}_{1}(-h)+2 \mu D \hat{v}_{1}(-h)-\epsilon E_{1} D \hat{\phi}_{1}(-h)-\frac{k^{2}}{C a} \hat{\zeta}_{1}\right) \\
\left(-\hat{p}_{2}(1)+2 D \hat{v}_{2}(1)-E_{2} D \hat{\phi}_{2}(1)\right)=0
\end{gathered}
$$

Finally, for layer 3, the x-momentum (2.3.12a), y-momentum (2.3.12b) and continuity $(2.3 .9 \mathrm{c})$ equations are expressed as:

$$
\begin{gathered}
R e_{1}\left(s \hat{u}_{3}\right)=-i k \hat{p}_{3}+\mu\left(D^{2}-k^{2}\right) \hat{u}_{3}, \\
\operatorname{Re}_{1}\left(s \hat{v}_{3}\right)=-D \hat{p}_{3}+\mu\left(D^{2}-k^{2}\right) \hat{v}_{3}, \\
i k \hat{u}_{3}+D \hat{v}_{3}=0,
\end{gathered}
$$

subject to the boundary and jump conditions:

$$
\begin{gathered}
\hat{u}_{3}(h)+D U_{3}(h) \hat{\zeta}_{2}=\hat{u}_{2}(-1)+D U_{2}(-1) \hat{\zeta}_{2}, \hat{u}_{1}(-h)=0, \\
\hat{v}_{3}(h)=\hat{v}_{2}(-1), \hat{v}_{3}(-h)=0,
\end{gathered}
$$


The perturbation potential Laplace equation for layer 2 is written as:

$$
\left(D^{2}-k^{2}\right) \hat{\phi}_{2}=0
$$

subject to the Ohmic current continuity for the top interface (from (2.3.15)),

$$
\sigma D \hat{\phi}_{1}(-h)=D \hat{\phi}_{2}(1)
$$

and Ohmic current continuity for the bottom interface (from (2.3.15)),

$$
D \hat{\phi}_{2}(-1)=\sigma D \hat{\phi}_{3}(h)
$$

The perturbation potential Laplace equation for layer 3 is written as:

$$
\left(D^{2}-k^{2}\right) \hat{\phi}_{3}=0
$$

subject to the zero perturbation at lower wall and from potential continuity at the bottom interface (2.3.14),

$$
\hat{\phi}_{3}(-h)=0,-E_{1} \hat{\zeta}_{2}+\hat{\phi}_{3}(h)=-E_{2} \hat{\zeta}_{2}+\hat{\phi}_{2}(-1),
$$

The tangential and normal stress matching conditions for the bottom interface 
are

$$
\begin{gathered}
\left(D \hat{u}_{2}(-1)+i k \hat{v}_{2}(-1)\right)+i k \epsilon E_{2}^{2} \hat{\zeta}_{2}-i k \epsilon E_{2} \hat{\phi}_{2}(-1) \\
-\left(\mu\left(D \hat{u}_{3}(h)+i k \hat{v}_{3}(h)\right)+i k \epsilon E_{1}^{2} \hat{\zeta}_{2}-i k \epsilon E_{1} \hat{\phi}_{3}(h)\right)=0
\end{gathered}
$$

$$
\begin{gathered}
\left(-\hat{p}_{2}(-1)+2 D \hat{v}_{2}(-1)-E_{2} D \hat{\phi}_{2}(-1)-\frac{k^{2}}{C a} \hat{\zeta}_{2}\right) \\
-\left(-\hat{p}_{3}(h)+2 \mu D \hat{v}_{3}(h)-\epsilon E_{1} D \hat{\phi}_{3}(h)\right)=0
\end{gathered}
$$

The inteface advection equation (2.2.8) becomes:

$$
\hat{v}=s \hat{\zeta}+i k U \hat{\zeta}
$$

i.e. for the top interface (with $\mathrm{U}=0$ ):

$$
\hat{\zeta}_{1}=\frac{\hat{v}_{2}(1)}{s}
$$

and for the bottom interface (with $\mathrm{U}=0$ ):

$$
\hat{\zeta}_{2}=\frac{\hat{v}_{2}(-1)}{s}
$$

(2.3.18a)-(2.3.28) are the set of perturbation equations that need to be solved to obtain the solution for perturbation variables. We attempt to solve these equations numerically using Chebyshev pseudospectral methods. 


\subsubsection{Chebyshev pseudospectral method}

The fundamental concept of a spectral method is to assume an approximation of the unknown variable $\mathrm{u}(\mathrm{x})$ by a sum of $\mathrm{N}+1$ basis functions $\phi_{n}(x)$ :

$$
u(x) \approx u_{N}(x)=\sum_{i=0}^{N} a_{n} \phi_{n}(x)
$$

Substituting this series into the equation of the form

$$
L u=f(x)
$$

where $\mathrm{L}$ is the operator of the partial differential equation, results in a "residual function" defined by

$$
R\left(x ; a_{0}, a_{1}, \ldots, a_{N}\right)=L u_{N}-f
$$

For the exact solution, the residual function $R\left(x ; a_{n}\right)$ is identically equal to zero. Hence, we need to choose the series coefficients $a_{n}$ so that the residual function is minimized. In the case of pseudospectral methods, the strategy for residual minimization is to make the residual zero at a set of points called "collocation" or "interpolation" points. Since the pseudospectral method evaluates the residual function only at the collocation points, the grid point values of the approximate solution, $u_{N}\left(x_{i}\right)$, are taken as the unknowns instead of the series coefficients. We then compute the $(\mathrm{N}+1)$ series coefficients $a_{n}$ through polynomial or trigonometric interpolation, having known the value of a function at $(\mathrm{N}+1)$ points. The 
error $\epsilon$ of a pseudospectral method is

$$
\epsilon \approx O\left[(1 / N)^{N}\right]
$$

The error decreases faster than any finite power of $\mathrm{N}$ since the power in the formula is always increasing. This is known as the "infinite order" or "exponential" convergence [48]. One of the most important basis functions used in (2.3.29) are the Chebyshev polynomials which are defined by

$$
T_{n}(\cos \theta)=\cos (n \theta)
$$

The Chebyshev pseudospectral method is a commonly used pseudo-spectral method for problems with non-periodic boundary conditions.

We solve the linearized three-layer electrohydrodynamic instability problem numerically using Chebyshev pseudospectral methods. The computational domain is mapped to $(-1,1)$ in each fluid layer, which is a standard procedure in numerical solution using Chebyshev polynomials. We follow the notation used in the "matrix differentiation suite" for MATLAB devised by Wiedemann and Reddy, 2000 [51]. Following their terminology, we suitably replace the differential operator "D" to its numerical counterparts " $D_{1}$ " in the first layer, " $D_{2}$ " in the second layer and " $D_{3}$ " in the third layer. Here, $D_{1}=D_{2} / h, D_{1}^{2}=D_{2}^{2} / h^{2}$, $D_{3}=D_{1}$ and $D_{2}$ is the standard differentiation assuming $-1 \leq \mathrm{y}^{*} \leq 1$. From the perturbation analysis performed in the previous subsection, we can see that there 
are totally 14 variables to be determined i.e. $\hat{u}_{i}, \hat{v}_{i}, \hat{p}_{i}, \hat{\phi}_{i}(i=1,2,3), \hat{\zeta}_{1}$ and $\hat{\zeta}_{2}$. The equations for the layers 1,2 and 3 are arranged suitably into matrices with the boundary conditions suitably incorporated to obtain the final form $A \mathbf{x}=\mathbf{b}$, where $\mathbf{x}$ is the set of 14 unknown variables. We refer the readers to [49], [50] and [51] for the relevant details. We then solve the matrix system to obtain the eigenvalues and eigenvectors of the matrix $A$. The eigenvalues provide us the growth rate values corresponding to the kink and sausage modes, while the eigenvectors contain the flow and electric variable perturbations corresponding to the kink and sausage modes.

\subsection{Results from pseudospectral method}

Firstly, we provide representative results from the pseudospectral method with regard to five important effects viz. - (1) Electrical property ratio effects, (2) depth ratio effects, (3) electric field effects, (4) surface-tension effects and (5) convective base flow effects. The influence of the afore-mentioned parameters on the stability (or, instability) of the kink and sausage modes would be addressed in the following sections. It is assumed that the density ratio equals 1 and the viscosity ratio is kept fixed as 0.284 . The experimental value of $1 \times 10^{-4} \mathrm{~N} / \mathrm{m}$ for the surface tension coefficient and a zero base flow velocity are used in all sections except for those where the effects of surface tension and convective base flow are studied. 


\subsubsection{Effects of electrical property ratios}

In the long-wave asymptotic analysis presented in Appendix A, it was deduced that the electrical properties play a crucial role in deciding the flow stability through their contributions to the tangential $\left(\left(Q_{T}\right)_{1}\right.$ and $\left.\left(Q_{T}\right)_{2}\right)$ and normal stress $\left(\left(Q_{N}\right)_{1}\right.$ and $\left.\left(Q_{N}\right)_{2}\right)$ continuity. Especially, the contributions of electrical properties to the total tangential stress $\left(Q_{T}\right)_{1}$ and $\left(Q_{T}\right)_{2}$ were found to be of utmost importance when $\epsilon=\sigma^{2}$. Another similar observation is that when $\epsilon=\sigma$, both $\left(\left(Q_{T}\right)_{1}\right.$ and $\left.\left(Q_{T}\right)_{2}\right)$ become zero implying a non-zero contribution from the $\left(\left(Q_{N}\right)_{1}\right.$ and $\left.\left(Q_{N}\right)_{2}\right)$ terms alone. In this section, we simplify the expressions of $\left(Q_{T}\right)_{1},\left(Q_{T}\right)_{2}$, $\left(Q_{N}\right)_{1}$ and $\left(Q_{N}\right)_{2}$ for kink $\left(\hat{s}_{1}=\hat{s}_{2}\right)$ and sausage $\left(\hat{s}_{1}=-\hat{s}_{2}\right)$ modes and illustrate the significance of electrical properties with respect to the results provided herein.

On substituting $\hat{s}_{1}=\hat{s}_{2}$ for the kink mode in (A.3.7), (A.3.9), (A.3.11) and (A.3.13), we get:

$$
\begin{aligned}
& \left(Q_{T}\right)_{1}=\frac{\hat{s}_{1}}{(1-\sigma-\eta)^{2}}\left\{\frac{\sigma(\epsilon-\sigma)(t h s h+c h)}{t h s h+\sigma c h}\right\}, \\
& \left(Q_{T}\right)_{2}=\frac{\hat{s}_{1}}{(1-\sigma-\eta)^{2}}\left\{\frac{\sigma(\sigma-\epsilon)(t h s h+c h)}{t h s h+\sigma c h}\right\}, \\
& \left(Q_{N}\right)_{1}=\frac{-\hat{s}_{1}}{(1-\sigma-\eta)^{2}}\left\{\frac{(\sigma-1)\left(\sigma^{2}-\epsilon\right) k s h}{t h s h+\sigma c h}\right\}, \\
& \left(Q_{N}\right)_{2}=\frac{-\hat{s}_{1}}{(1-\sigma-\eta)^{2}}\left\{\frac{(\sigma-1)\left(\sigma^{2}-\epsilon\right) k s h}{t h s h+\sigma c h}\right\},
\end{aligned}
$$

from which we observe that $\left(Q_{T}\right)_{1}$ and $\left(Q_{T}\right)_{2}$ are equal in magnitude but opposite in sign while $\left(Q_{N}\right)_{1}$ and $\left(Q_{N}\right)_{2}$ are equal for the kink mode. Proceeding similarly 
with $\hat{s}_{1}=-\hat{s}_{2}$ for the sausage mode, we obtain:

$$
\begin{aligned}
& \left(Q_{T}\right)_{1}=\frac{\hat{s}_{1}}{(1-\sigma-\eta)^{2}}\left\{\frac{\sigma(\epsilon-\sigma)(t h c h+s h)}{t h c h+\sigma s h}\right\}, \\
& \left(Q_{T}\right)_{2}=\frac{\hat{s}_{1}}{(1-\sigma-\eta)^{2}}\left\{\frac{\sigma(\epsilon-\sigma)(t h c h+s h)}{t h c h+\sigma s h}\right\}, \\
& \left(Q_{N}\right)_{1}=\frac{-\hat{s}_{1}}{(1-\sigma-\eta)^{2}}\left\{\frac{(\sigma-1)\left(\sigma^{2}-\epsilon\right) k c h}{t h c h+\sigma s h}\right\}, \\
& \left(Q_{N}\right)_{2}=\frac{\hat{s}_{1}}{(1-\sigma-\eta)^{2}}\left\{\frac{(\sigma-1)\left(\sigma^{2}-\epsilon\right) k c h}{t h c h+\sigma s h}\right\},
\end{aligned}
$$

noting that $\left(Q_{T}\right)_{1}=\left(Q_{T}\right)_{2}$ and $\left(Q_{N}\right)_{1}=-\left(Q_{N}\right)_{2}$ for the sausage mode. It can also be inferred that the signs of $\left(Q_{N}\right)_{1}$ and $\left(Q_{N}\right)_{2}$ in both the modes change depending on whether $\epsilon>\sigma^{2}$ or $\epsilon<\sigma^{2}$ meaning that the normal stress acting across the interface can have different directions based on the values of $\epsilon$ and $\sigma^{2}$. It is also worth pointing out from the expressions of $\left(Q_{N}\right)_{1}$ and $\left(Q_{N}\right)_{2}$ that it is not just the value of " $\left(\sigma^{2}-\epsilon\right)$ ", but the product " $(\sigma-1)\left(\sigma^{2}-\epsilon\right)$ " which plays a crucial role in stabilizing or destabilizing the flow. This observation has been reported earlier in Ozen et al. [15] albeit for the case of a single interface undergoing EHD instability. We consider 5 different cases of interest for results to further substantiate the inferences above: (a) $\sigma^{2}>\epsilon$ and $\sigma>1$ (Case A), (b) $\sigma^{2}<\epsilon$ and $\sigma<1$ (Case B), (c) $\sigma^{2}>\epsilon$ and $\sigma<1$ (Case C), (d) $\sigma^{2}<\epsilon$ and $\sigma>1$ (Case D) and (e) $\sigma^{2}=\epsilon$ (Case E). It can be easily seen that for cases A and B (denoted together as "Type-I" from now on for convenience), the product " $(\sigma-1)\left(\sigma^{2}-\epsilon\right)$ " is positive, for the cases $\mathrm{C}$ and $\mathrm{D}$ ("Type-II") it is negative while for case E ("Type-III") it is zero. The depth ratio $n$ is taken to 


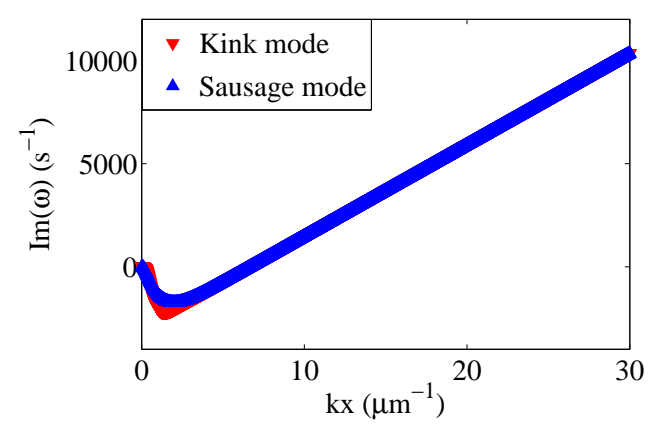

(a) Case $\mathrm{A}\left(\sigma^{2}>\epsilon\right.$ and $\left.\sigma>1\right)$.

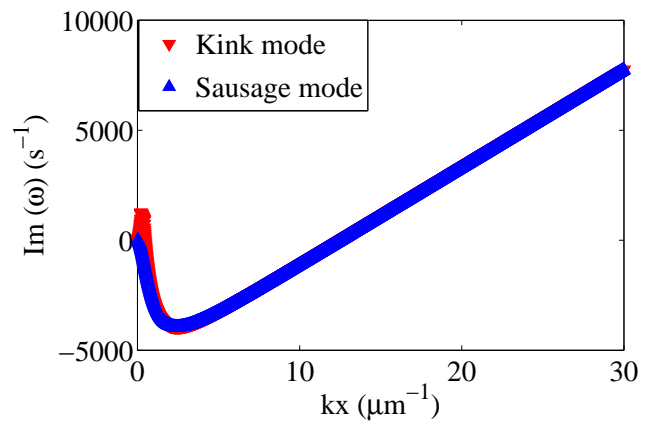

(b) Case B $\left(\sigma^{2}<\epsilon\right.$ and $\left.\sigma<1\right)$.

Figure 2.2: The plot of imaginary part of dimensional growth rate $(\operatorname{Im}(\omega))$ vs. the dimensional wavenumber $(\mathrm{kx})$ for cases $\mathrm{A}$ and B (Type-I) for which the product $(\sigma-1)\left(\sigma^{2}-\epsilon\right)$ is positive. Higher growth rates for both modes compared to cases $\mathrm{C}$ and D (Type-II) are predicted primarily because the electrical properties considered have a destabilizing effect on the flow. In figure (a), $\sigma=10^{1}, \epsilon=10^{1}$ and in figure (b) $\sigma=10^{-0.5}, \epsilon=10^{1}$ have been used.

be equal to 1 . The imaginary part of dimensional growth rate $(\operatorname{Im}(\omega))$ is plotted against the dimensional wavenumber $(\mathrm{kx})$ for the cases $\mathrm{A}$ and $\mathrm{B}$ in figure 2.2.

The growth rate versus wavenumber plot for the cases $\mathrm{C}$ and $\mathrm{D}$ are provided in figure 2.3. By comparing the growth rate results in figures 2.2 and 2.3 , it can be observed that for both kink and sausage modes, the maximum growth rates corresponding to type-I are much higher compared to those of type-II and concluded that the electrical properties are expected to have a destabilizing effect when the product " $(\sigma-1)\left(\sigma^{2}-\epsilon\right)$ " is positive and stabilizing effect when the product is negative. This is in agreement with the conclusion of Ozen et al. [23] for an interface sandwiched between two immiscible fluids and can be regarded as an extension to a two-interface case having two distinct modes of perturbation (kink and sausage). It can also be noted from figure 2.2 that the kink mode has 


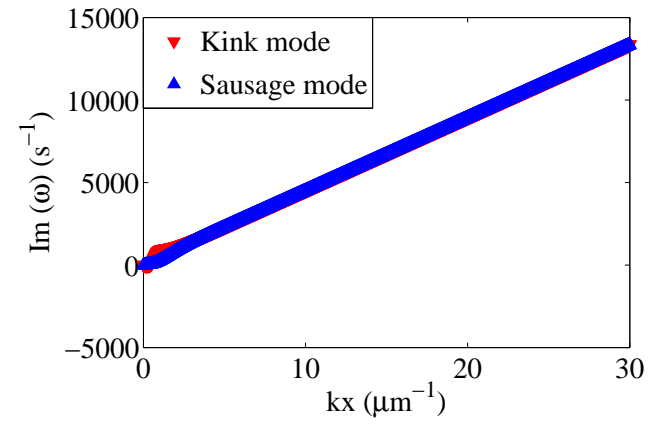

(a) Case $\mathrm{C}\left(\sigma^{2}>\epsilon\right.$ and $\left.\sigma<1\right)$.

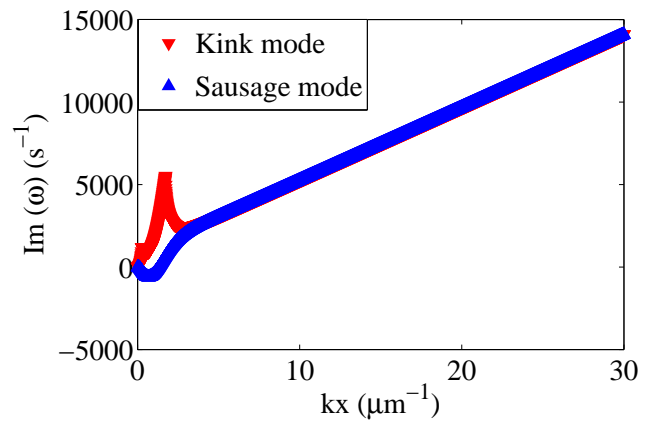

(b) Case $\mathrm{D}\left(\sigma^{2}<\epsilon\right.$ and $\left.\sigma>1\right)$.

Figure 2.3: The plot of imaginary part of dimensional growth rate $(\operatorname{Im}(\omega))$ vs. the dimensional wavenumber $(\mathrm{kx})$ for cases $\mathrm{C}$ and $\mathrm{D}$ (Type-II) for which the product $(\sigma-1)\left(\sigma^{2}-\epsilon\right)$ is negative. Lower growth rates for both modes compared to cases A and B (Type-I, figure 2.2) can be observed. The electrical properties considered are said to have a stabilizing effect on the flow. In figure (a) $\sigma=10^{-0.5}, \epsilon=10^{-1.5}$ and in figure (b) $\sigma=10^{0.25}, \epsilon=10^{1}$ are used.

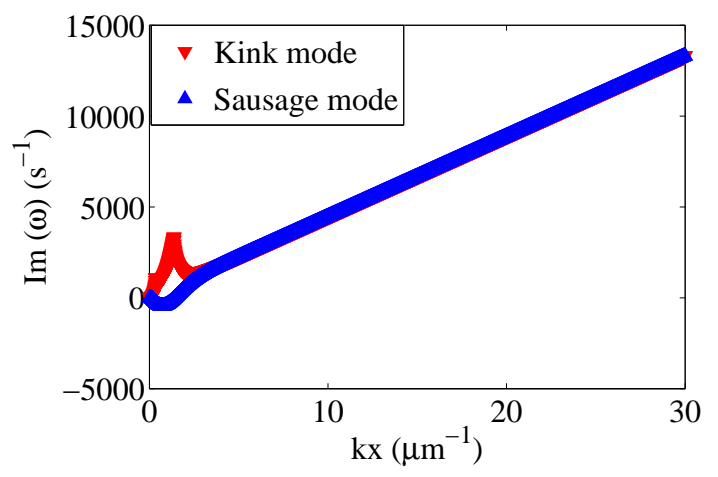

Figure 2.4: The plot of imaginary part of dimensional growth rate $(\operatorname{Im}(\omega))$ vs. the dimensional wavenumber $(\mathrm{kx})$ for cases $\mathrm{E}$ (Type-III) for which the product $(\sigma-1)\left(\sigma^{2}-\epsilon\right)$ is zero. The stability/instability of the flow is dictated by the tangential stress contributions $\left(Q_{T}\right)_{1}$ and $\left(Q_{T}\right)_{2}$ alone. The values of $\sigma=10^{0.5}$, $\epsilon=10^{1}$ are used. 


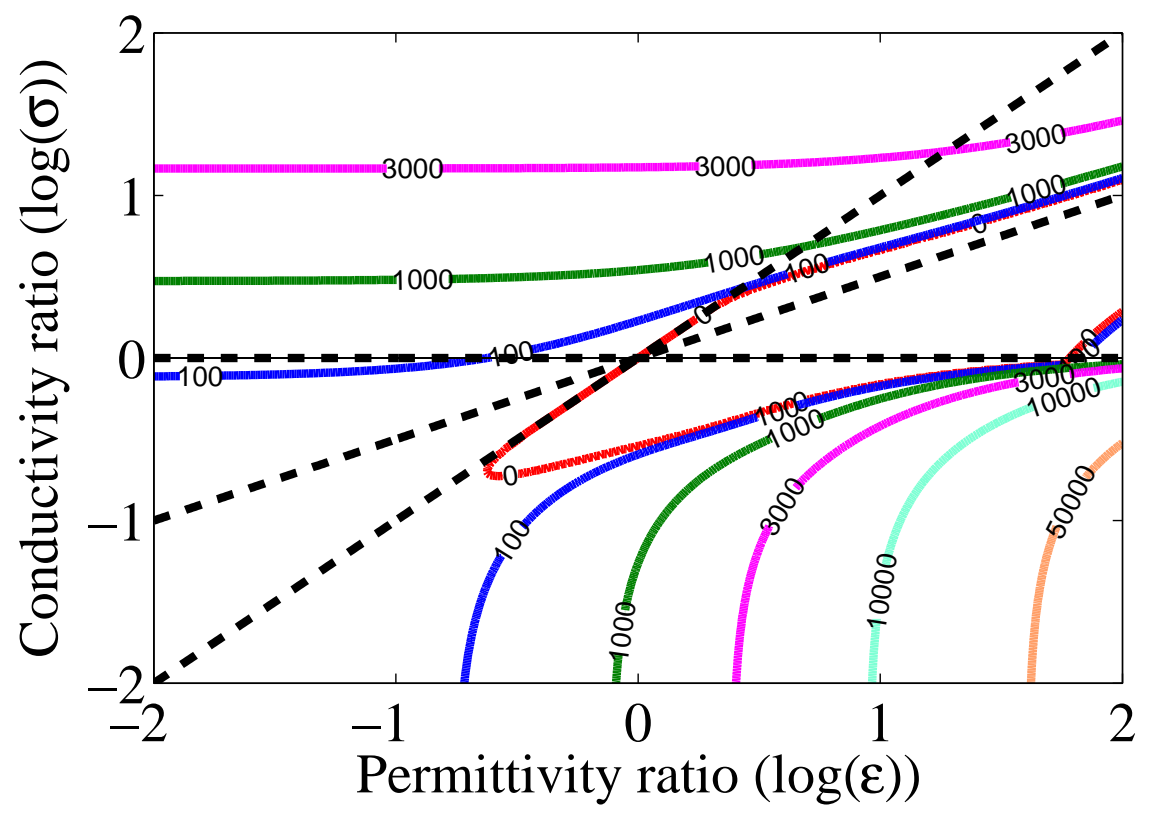

Figure 2.5: Log-log plot of electrical conductivity ratio $(\sigma)$ against dielectric permittivity ratio $(\epsilon)$ depicting maximum growth rate values for the case of kink mode with the depth ratio $n=1$. The neutral stability curve (red) and other constant growth rate curves have been plotted. Three dashed lines corresponding to $\epsilon=\sigma, \epsilon=\sigma^{2}$ and $\sigma=1$ are also drawn to highlight the regions pertaining to types I and II.

slightly higher maximum growth rates than the sausage mode for case A while the contrary is true for case B. For the case C, the growth rates of short waves for the kink mode are slightly negative (and hence, unstable) while the sausage mode is extremely stable (growth rates are positive). On the other hand, the kink mode is stable while sausage mode has small negative growth rates in the short wave region for the case D (figure 2.3). Furthermore, the results for case $\mathrm{E}$ in figure 2.4 are similar to those observed for case D except that the growth rates for the sausage mode are slightly higher for case D compared to case E. Of course, these inferences are true for the electric property ratios specifically chosen for each of 


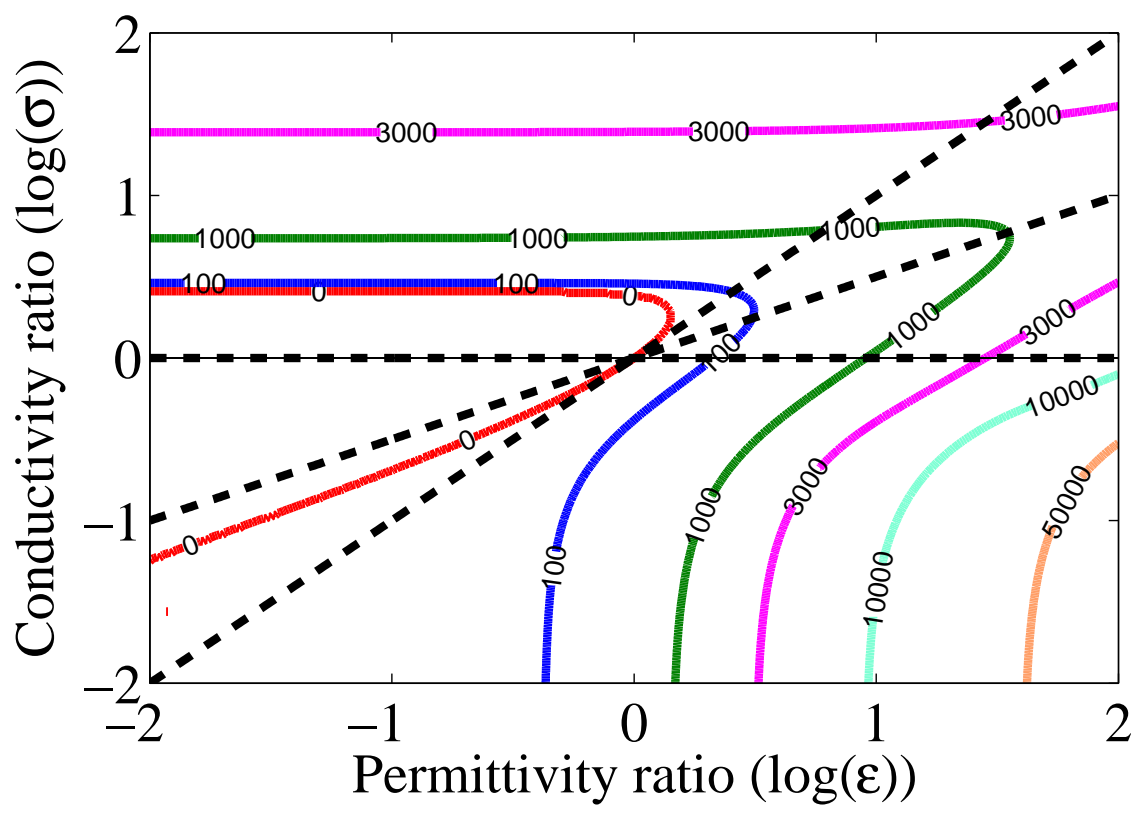

Figure 2.6: Log-log plot of electrical conductivity ratio $(\sigma)$ against dielectric permittivity ratio $(\epsilon)$ with maximum growth rate values for the case of sausage mode with the depth ratio $n=1$. The neutral stability curve (red), a few constant growth rate curves and the three dashed lines corresponding to $\epsilon=\sigma, \epsilon=\sigma^{2}$ and $\sigma=1$ are plotted.

these cases and a more detailed analysis by varying these ratios is required for generalization.

Although the particular values of $\epsilon$ and $\sigma$ chosen in cases A-E above provide some basic qualitative understanding, it would be worthwhile to have a growth rate plot for a certain range of permittivities and conductivities. Uguz et al. [25] presented such a plot for the single interface case to mark regions with stabilizing or destabilizing effects of parallel or perpendicularly applied electric fields, albeit for a very narrow range of electrical property values (omitting very low values). In this work, we provide a detailed log-log plot for electrical conductivity 
and dielectric permittity values each ranging between $10^{-2}$ to $10^{2}$ and the corresponding maximum growth rate value for each possible combination. In this plot, the neutral stability curve (zero growth rate) and certain other constant growth rate regions are marked thereby clearly demarcating stable and unstable regions. Such a plot would provide valuable information about growth rate characteristics in different regions corresponding to cases A-D. Though the stability regions are altered with change in depth ratio $n$, applied electric field and mechanical property ratios, the plots in this section address the case where depth ratio $n=1$, density ratio $\rho=1$, viscosity ratio $\mu=0.284$ and applied electric field $\mathrm{E}=3 \times 10^{5} \mathrm{~V} / \mathrm{m}$. The plots for kink and sausage modes are given in figures 2.5 and 2.6 respectively. Three dashed lines marking $\epsilon=\sigma, \epsilon=\sigma^{2}$ and $\sigma=1$ can also be seen. For the kink mode, as predicted earlier, the most stable region (the one inside the neutral stability curve) belongs predominantly to the one where the product $(\sigma-1)\left(\sigma^{2}-\epsilon\right)$ is negative. The lowest growth rates are observed in the left hand corner of the third quadrant $(\epsilon<<1$ and $\sigma<<1)$ while the growth rates in the right hand corner of the fourth quadrant $(\epsilon>>\sigma)$ are the highest. Lower growth rates are observed on the $\epsilon=\sigma^{2}$ line compared to those slightly away from the line. The sausage mode plot depicts slightly different features compared to the kink mode, although certain similarites can be deduced. The similarities include (a) the stable region being associated with Type-II characteristics, (b) lowest and highest growth rates in the corners of third and four quadrants respectively and (c) lower growth rates on the $\epsilon=\sigma^{2}$ line. The major differences are: (a) the stable region is observed in the third quadrant and (b) relatively higher growth rates are seen in 


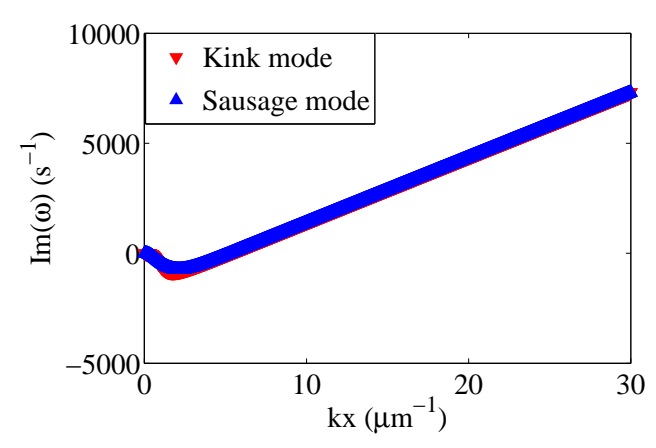

(a) Case $\mathrm{A}\left(\sigma^{2}>\epsilon\right.$ and $\left.\sigma>1\right)$.

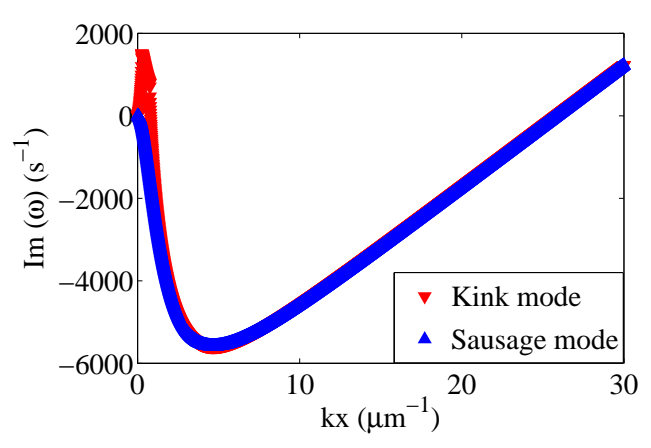

(b) Case B $\left(\sigma^{2}<\epsilon\right.$ and $\left.\sigma<1\right)$.

Figure 2.7: The plot of imaginary part of dimensional growth rate $(\operatorname{Im}(\omega))$ vs. the dimensional wavenumber $(\mathrm{kx})$ for cases A and B (Type-I) and the depth ratio $n$ equals 0.5 . Higher growth rates for both modes compared to cases $\mathrm{C}$ and $\mathrm{D}$ (Type-II) are predicted as the electrical properties tend to destabilize the flow. In figure (a), $\sigma=10^{1}, \epsilon=10^{1}$ and in figure (b) $\sigma=10^{-0.5}, \epsilon=10^{1}$.

the first quadrant region obeying Type-II characteristics. An interesting finding is that the $\epsilon=\sigma^{2}$ line divides the plot almost symmetrically with growth rates increasing along the line from the third quadrant to the first quadrant.

\subsubsection{Effects of depth ratio}

In the previous section, results were presented for the case where the three layers were of equal thickness i.e. $n=1$. Quite obviously, the kink and sausage mode characteristics would be quite different for a different depth ratio. Based on the analytical expression obtained from their long-wave analysis for a single interface, Li et al. [24] explained the importance of depth ratio using cases where the viscosity ratio $\mu>n^{2}$ and $\mu<n^{2}$. We temporarily consider these cases as the derivation of analytical solution for long-wave behaviour of three-layer instability 
will be undertaken in future. With the viscosity ratio being fixed at $\mu=0.284$, the depth ratio $n=1$ belongs to $\mu<n^{2}$ category. In this section, the sandwiched layer is assumed to be twice as much thick as the sandwiching layers. This implies a depth ratio of $n=0.5$ and hence belongs to $\mu>n^{2}$ category. Even though a detailed log-log plot as provided earlier for $n=1$ is still a work in progress, some exemplary results are provided for cases A-D. The growth rate plot for the cases $\mathrm{A}$ and $\mathrm{B}$ is provided in figure 2.7. On comparing with $n=1$ results for cases $\mathrm{A}$ and $\mathrm{B}$ in figure 2.2, no significant changes in the growth rate pattern is observed. But the maximum growth rate values are higher (approximately twice as high for case A) for $n=0.5$. From comparison of the growth rate plots for cases $\mathrm{C}$ and $\mathrm{D}$ in figures 2.8 and 2.3, the maximum growth rate for the kink mode with $n=0.5$ is found to be twice as that for $n=1$ while there is an order of magnitude (about thirty times) reduction in the maximum growth rate for the sausage mode with $n=0.5$.

\subsubsection{Effects of electric field}

The flow stability behaviour can be altered significantly by varying the applied electric field. Though it is expected that increasing the electric field would result in increase in growth rates, we shall provide examples to show that this is not always the case. Kink and sausage mode results for cases A and D are presented in this section. The capillary number "Ca" depends on the electro-viscous velocity and in turn, on the square of the applied electric field. The depth ratio $n$ is 


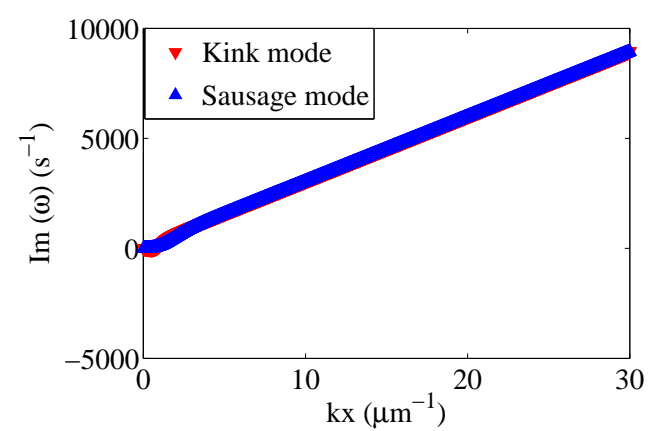

(a) Case $\mathrm{C}\left(\sigma^{2}>\epsilon\right.$ and $\left.\sigma<1\right)$.

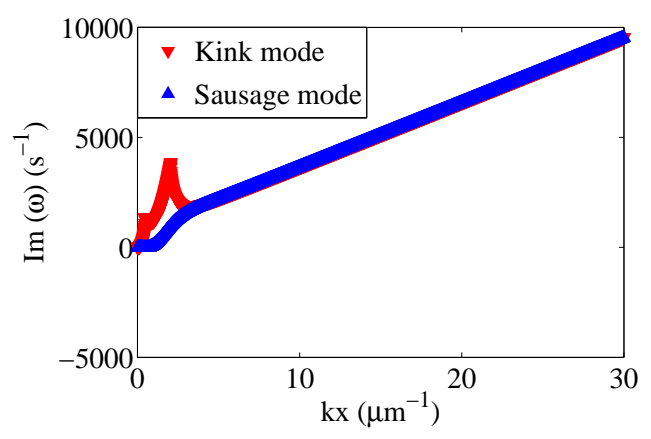

(b) Case $\mathrm{D}\left(\sigma^{2}<\epsilon\right.$ and $\left.\sigma>1\right)$.

Figure 2.8: The plot of imaginary part of dimensional growth rate $(\operatorname{Im}(\omega))$ vs. the dimensional wavenumber $(\mathrm{kx})$ for cases $\mathrm{C}$ and $\mathrm{D}$ (Type-II) and the depth ratio $n$ equals 0.5 . Lower growth rates for both modes compared to cases $\mathrm{A}$ and B (Type-I, figure 2.2) can be observed. The electrical properties are said to have a stabilizing effect on the flow. In figure (a) $\sigma=10^{-0.5}, \epsilon=10^{-1.5}$ and in figure (b) $\sigma=10^{0.25}, \epsilon=10^{1}$ are used.

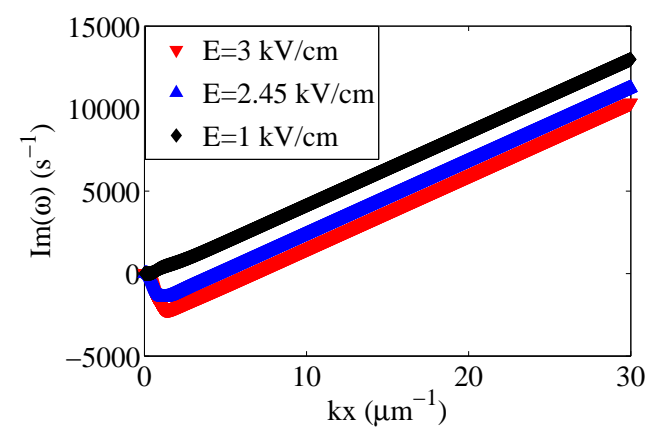

(a) Kink mode (Case A).

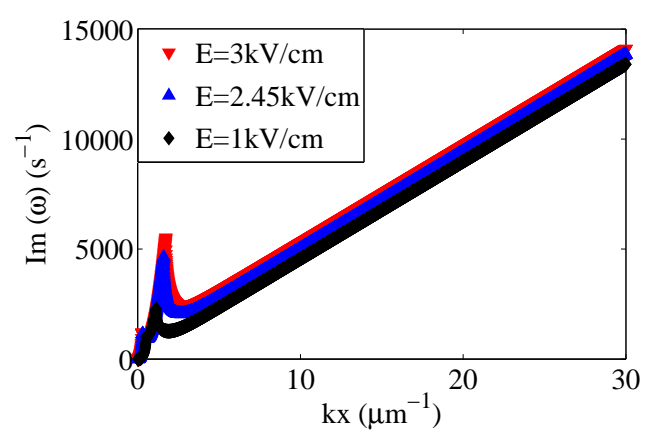

(b) Kink mode (Case D).

Figure 2.9: The growth rate plot for kink mode perturbation pertaining to cases $\mathrm{A}$ and $\mathrm{D}$ with various electric field values. The specific values of $\sigma=10^{1}, \epsilon=10^{1}$ and $\sigma=10^{0.25}, \epsilon=10^{1}$ are taken for figures (a) and (b) respectively. With increasing electric field, flow tends to be more unstable with higher growth rates for case A. An opposite effect is observed for case D. 


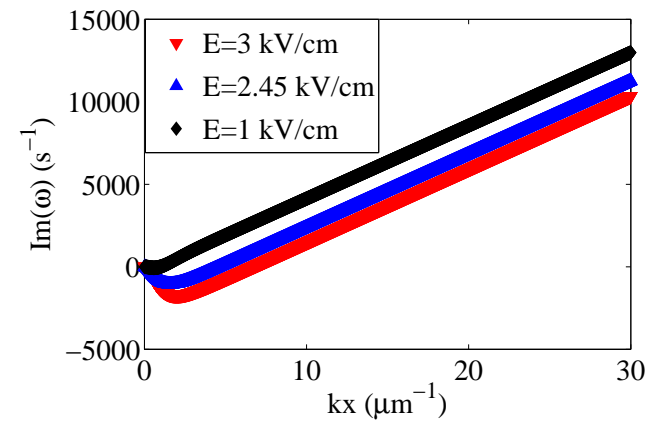

(a) Sausage mode (Case A).

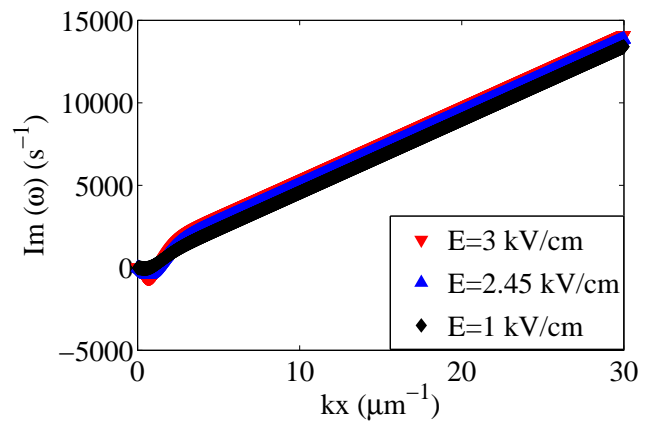

(b) Sausage mode (Case D).

Figure 2.10: The growth rate plot for sausage mode perturbation pertaining to cases $\mathrm{A}$ and $\mathrm{D}$ with various electric field values. Same parametric values as figure 2.9 are used. An anologous behaviour as in the case of kink mode (figure 2.9) has been observed except that for case D, growth rates increase with increase in electric field for low wavenumbers.

taken to be equal to 1 . Three values of electric field: $\mathrm{E}=3 \times 10^{5} \mathrm{~V} / \mathrm{m}(\mathrm{Ca}=1.45)$, $\mathrm{E}=2.49 \times 10^{5} \mathrm{~V} / \mathrm{m}(\mathrm{Ca}=1)$ and $\mathrm{E}=1 \times 10^{5} \mathrm{~V} / \mathrm{m}(\mathrm{Ca}=0.16)$ have been considered. The growth rate plots for the kink mode perturbation related to cases A and D are provided in figure 2.9. As the electric field is increased, the flow stability is considerably reduced for case A (figure 2.9(a)) resulting in higher maximum growth rates and a higher fastest growth rate wavenumber. On the contrary, stability is enhanced for case D (figure 2.9(b)) with higher electric fields. For the sausage mode, a very similar qualitative behaviour as the kink mode is observed for cases A and D, as can be seen in figure 2.10. A slight difference which can be observed for case D (figure $2.10(\mathrm{~b})$ ) is that the growth rates of low wavenumbers are reduced while the high wavenumbers tend to become slightly more unstable 


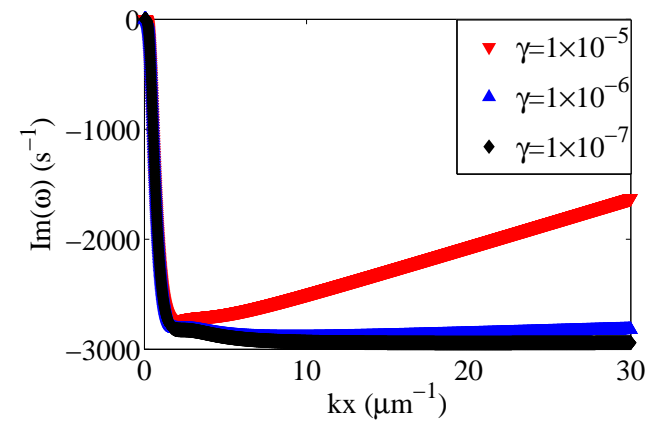

(a) Kink mode.

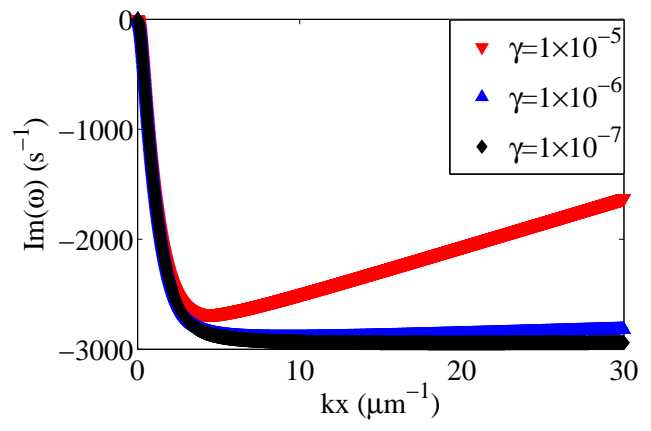

(b) Sausage mode.

Figure 2.11: The growth rate plot for case A and various surface tension coefficients. The decrease in surface tension results in failure to stabilize the waves with high wavenumber (or small wavelength) resulting in higher growth rate values and a wider range of fastest growth rate wavenumbers. This trend has been observed for both kink (figure (a)) and sausage (figure (b)) modes. The values of $\sigma=10^{1}$ and $\epsilon=10^{1}$ were used.

with decreasing electric field. These results indicating opposite effects of increasing electric fields depending on the electrical properties (cases A and D) are in agreement with those of single interface case in Li et al. [24].

\subsubsection{Effects of surface tension}

From the long-wave asymptotic analysis in Appendix A, it can be noticed that surface tension effects do not contribute to terms first-order or lower and hence do not affect the stability behaviour of long waves. Nonetheless, surface tension effects have a third-order contribution and lead to stabilization of short waves. Hence with reduction of surface tension (say, with the help of surfactants), short waves would tend to become more unstable with significantly higher growth rates and it might lead to a wider range of wavenumbers corresponding to the fastest 


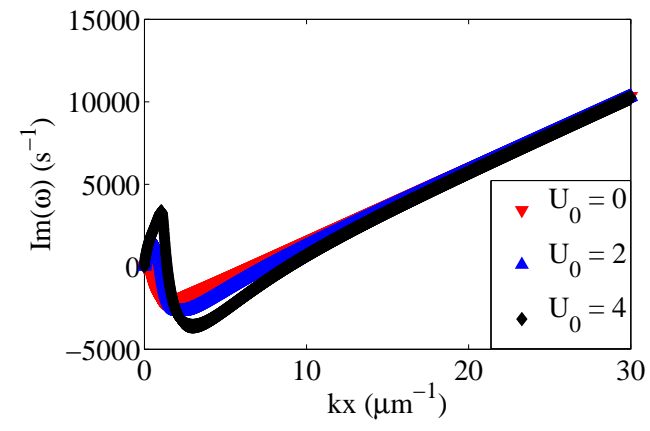

(a) Kink mode (Case A).

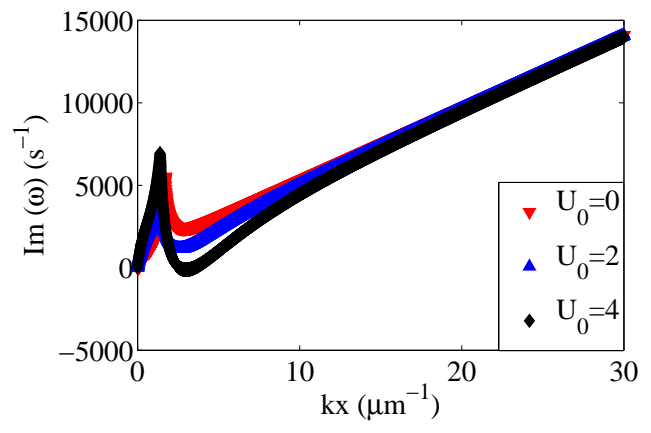

(b) Kink mode (Case D).

Figure 2.12: The growth rate plot for kink mode and various superposed baseflow values (user-defined parameter $U_{c}$ ). Long wave growth is suppressed while short wave growth rates increase with increase in the user-defined parameter $U_{c}$ (pressure gradient). The fastest growth rate wavenumbers increase correspondingly. For case $\mathrm{A}, \sigma=10^{1}$ and $\epsilon=10^{1}$ were used while for case $\mathrm{D}, \sigma=10^{0.25}$ and $\epsilon=10^{1}$ were used.

growth rates. This is exactly what can be observed for the cases of $\gamma=1 \times$ $10^{-6} \mathrm{~N} / \mathrm{m}$ and $\gamma=1 \times 10^{-7} \mathrm{~N} / \mathrm{m}$ in figure 2.11 where the growth rate results are plotted for three different values of surface tension coefficient $(\gamma)$. Both kink and sausage modes exhibit this behaviour immaterial of the electrical property values (other cases not shown for brevity). The depth ratio $n$ is taken as 1 and the applied electric field is $\mathrm{E}=3 \times 10^{5} \mathrm{~V} / \mathrm{m}$.

\subsubsection{Effects of convective base flow}

In all the results mentioned above, the base flow was assumed to be "quiescent". Now, we examine the effects of a superposed plane-Poiseuille base flow on the flow stability. In particular, the growth rates are compared for three different values of the user-defined parameter $U_{c}$ (refer to chapter 2 for details): $U_{c}=0$, 


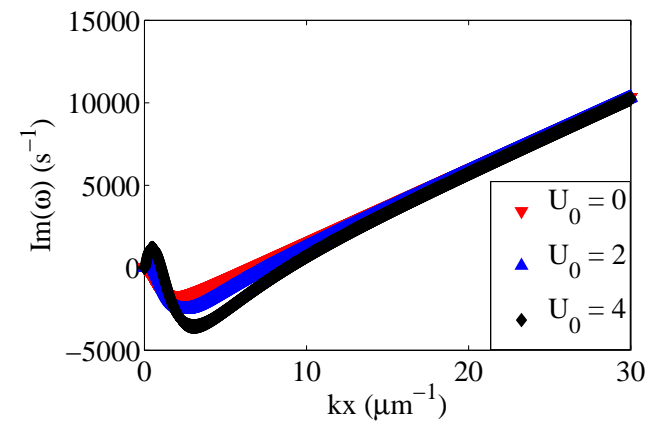

(a) Sausage mode (Case A).

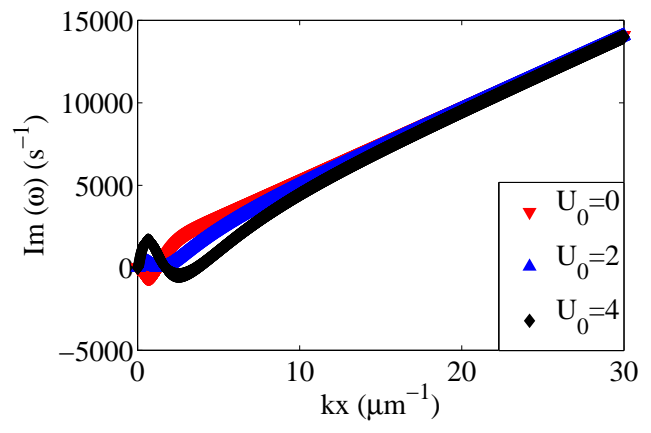

(b) Sausage mode (Case D).

Figure 2.13: The growth rate plot for sausage mode and various superposed baseflow values (user-defined parameter $U_{c}$ ). Same parametric ratios as figure 2.12 are considered. Similar conclusion as that for the kink mode applies for the sausage mode.

$U_{c}=2$ and $U_{c}=4$. Kink and sausage mode results are presented in figures 2.12 and 2.13 respectively. To detect any contribution from the electrical property ratios towards alteration of stability characteristics, cases A and D are considered. The depth ratio $n$ equals 1 and the applied electric field is $\mathrm{E}=3 \times 10^{5} \mathrm{~V} / \mathrm{m}$. First considering kink mode and comparing the results for cases A and D in figure 2.12, we note that increasing the parameter $U_{c}$ (and hence the dimensionless pressure gradient), the long waves are stabilized while the short waves are destabilized. The growth rates of the short waves increase and so does the fastest growth rate wavenumber. Contrary to the results for electric field effects, the there does not seem to be any change in trend for cases A and D. The same inference can be drawn from the results of cases $\mathrm{A}$ and $\mathrm{D}$ shown in figure 2.13 corresponding to the sausage mode. 


\section{Chapter 3}

\section{Fully nonlinear simulation}

In this section, the details of the computational methods utilized to simulate the nonlinear electrohydrodynamic instability in a three-layer viscous stratified flow are discussed. Surface tension effects would be neglected temporarily, though it is straightforward to add using the numerical technique considered. The primary reason for neglecting surface tension is that for the parameters to be considered in the simulations, it was verified from the linear stability analysis that the error due to neglection of surface tension effects resulted in an error of less than about $5 \%$ for the growth rate results (See chapter 4 for more details). This would be relaxed in the future simulations and surface tension effects can be easily included using the front-tracking method. We also consider the presence of a pressure-gradient based convective base flow (Plane-Poiseuille).

\subsection{Nonlinear simulation methodology}

\subsubsection{Governing equations}

The schematic for the problem was shown in the previous section (figure 2.1). The channel is assumed to be infinitely long in the $X$-direction and hence, we 
incorporate periodic boundary conditions in the $X$-direction to reduce the size of the computational domain. We consider the channel width to be $2 H$ and channel length to be $2 \pi H$, with an aspect ratio of $\pi$. The fluids under consideration have different densities $(\rho)$, viscosities $(\mu)$, electrical conductivities $(\sigma)$ and dielectric permittivities $(\epsilon)$. The governing equations for fluid flow are given by:

$$
\begin{aligned}
\nabla \cdot \mathbf{u} & =0, \text { and } \\
\rho\left(\frac{\partial \mathbf{u}}{\partial t}+\mathbf{u} \cdot \nabla \mathbf{u}\right) & =-\nabla p+\nabla \cdot\left[\mu\left(\nabla \mathbf{u}+\nabla \mathbf{u}^{T}\right)\right]+\mathbf{F}_{e}
\end{aligned}
$$

Here, we consider a body-force term in the flow equations, $\mathbf{F}_{e}(\mathbf{x}, t)$, due to the interaction of conductivity and permittivity gradients with the electric field. The electric body force $\mathbf{F}_{\mathbf{e}}$ can be expressed as $\mathbf{F}_{\mathbf{e}}=\rho_{E} \mathbf{E}-\frac{1}{2} \epsilon|\mathbf{E}|^{2}$ (Melcher and Taylor 1969), where $\rho_{E}$ represents the charge density. The inclusion of electric body force $\mathbf{F}_{e}$ term on the right hand side is a standard procedure in front-tracking method in which the interfacial stresses are distributed onto the Cartesian grids, and treated as body forces in the Navier-Stokes equations. The use of a single flow field variable $\mathbf{u}$ is a consequence of the "one-fluid" formulation [37]. The electric potential field and hence the electric field required to obtain the body force term $\mathbf{F}_{e}$ are obtained by solving the Ohmic equation:

$$
\nabla \cdot(\sigma \nabla \Phi)=0
$$


Here $\sigma(\mathbf{x}, t)$ is the electrical conductivity in the entire fluid: in the sandwiched layer, $\sigma=\sigma_{2}$, and for sandwiching layers, $\sigma=\sigma_{1}$. Similar definitions hold for the density, viscosity and permittivity variables. The interfaces advect with the local fluid velocity:

$$
\frac{d \mathbf{x}}{d t}=\mathbf{u}(\mathbf{x})
$$

where, $\mathbf{x}$ represents the interface coordinates.

\subsubsection{Scaling and dimensionless parameters}

We use the channel half-width $(\mathrm{H})$ as the length scale and the electro-viscous velocity $U_{e v}=\epsilon_{2} E_{0}^{2} H / \mu_{2}$ for scaling the velocity. The pressure is scaled by $p^{*}=\rho U_{e v}^{2}$. The fluid properties are scaled with respect to the properties of the sandwiched (organic) fluid. The constant applied potential $V_{0}$ and constant applied electric field $E_{0}$ are used to scale electric potential and electric field respectively. The most important dimensionless parameter is the Reynolds number

$$
R e=\frac{\rho_{2} U_{e v} H}{\mu_{2}}
$$

\subsubsection{Numerical solution technique}

The simulation technique employed to track the interfaces is the front-tracking / immersed boundary method (Peskin [39], Unverdi \& Tryggvason [38], Tryggvason et al. [37]) for multiphase flows. The chief characteristic of the front tracking method is the usage of a single set of equations for both the phases called the 
"one-fluid" formulation. Such a formulation implicitly accounts for the requisite interface jump conditions and it can be verified that this formulation contains the equations written separately for each fluid and the jump conditions. An Eulerian (fixed) grid for solving the flow variables and a Lagrangian (moving) grid for tracking the fluid-fluid interfaces are employed. As the interface front deforms, the fluid properties need to be updated suitably. In the front tracking method, the two different fluids are identified using an indicator (Heaviside) function and the interfaces between them are marked by a nonzero value of the gradient of indicator function. In our case, the indicator function is assigned a value of 1 for the aqueous fluid and 0 for the organic fluid. It was verified that the choice of the values for aqueous and organic fluids had no effect on the numerical solution. The gradient of indicator function $\mathbf{G}$ is defined as:

$$
\mathbf{G}=-\int_{S} \delta^{2}\left(\mathbf{x}-\mathbf{x}^{\prime}\right) \mathbf{n}^{\prime} d s^{\prime}
$$

where, $\mathrm{S}$ represents the interface contour, $\delta^{2}$ is the $2 \mathrm{D}$ dirac delta function and $\mathbf{n}^{\prime}$ is the unit normal to the interface. The $\delta$ function used in (3.1.6) is the product of two $1 \mathrm{D} \delta$ functions:

$$
\delta^{2}\left(\mathbf{x}-\mathbf{x}^{\prime}\right)=\delta\left(x-x^{\prime}\right) \delta\left(y-y^{\prime}\right)
$$

Peskin's distribution function $D$ is commonly utilized as the discrete and smooth form of Dirac-delta function $\delta$ in the front tracking method, whose numerical form 
is given as

$D\left(\mathbf{x}-\mathbf{x}^{\prime}\right)=\frac{1}{16 \Delta^{2}} \prod_{i=1}^{2}\left(1+\cos \frac{\pi}{2 \Delta}\left(x_{i}-x_{i}^{\prime}\right)\right) \quad$ for $\quad\left|x_{i}-x_{i}^{\prime}\right| \leq 2 \Delta, \quad i=1,2$, $D\left(\mathbf{x}-\mathbf{x}^{\prime}\right)=0 \quad$ otherwise,

where, $\Delta$ is the Eulerian grid size. The Peskin's distribution function $D$ imparts a finite thickness to the interface and the fluid properties vary smoothly over a circle of diameter $2 \Delta$ or roughly four fixed grid points close to the actual interface positions. Knowing $\mathbf{G}$, the indicator function $I(\mathbf{x})$ can be obtained by solving the following Poisson equation:

$$
\nabla^{2} I=\nabla \cdot \mathbf{G}
$$

Once the indicator function has been obtained, the fluid properties, like electrical conductivity for example, are updated as:

$$
\sigma(\mathbf{x})=\sigma_{2}+\left(\sigma_{1}-\sigma_{2}\right) I(\mathbf{x})
$$

\subsubsection{Staggered grid}

Before proceeding to discuss the methodology for solving the flow equations, we discuss briefly the finite-volume spatial discretization of the governing partial differential equations in the flow domain. Staggered grids offer enormous advantages, like stability and better conservation properties, over the co-located grids. 
The control volumes for the $\mathrm{u}$ velocity, $\mathrm{v}$ velocity and pressure are depicted in figure 3.1, figure 3.2 and figure 3.3 respectively. For the 2D problem considered, the points at which the velocity in the $\mathrm{x}$-direction $(\mathrm{u})$ is calculated are indicated by circles (o), the points at which the velocity in the y-direction (v) is calculated are indicated by crosses $(\times)$ and the points at which the pressure $(p)$ is calculated are indicated by dark dots $(\bullet)$. The fluid properties like density, viscosity, dielectric permittivity and conductivity are calculated on the pressure points, the electric potential is calculated on the pressure points, while the electric field in the x-direction $\left(E_{x}\right)$ is calculated in the u-velocity points and the electric field in the y-direction $\left(E_{y}\right)$ is calculated in the v-velocity points.

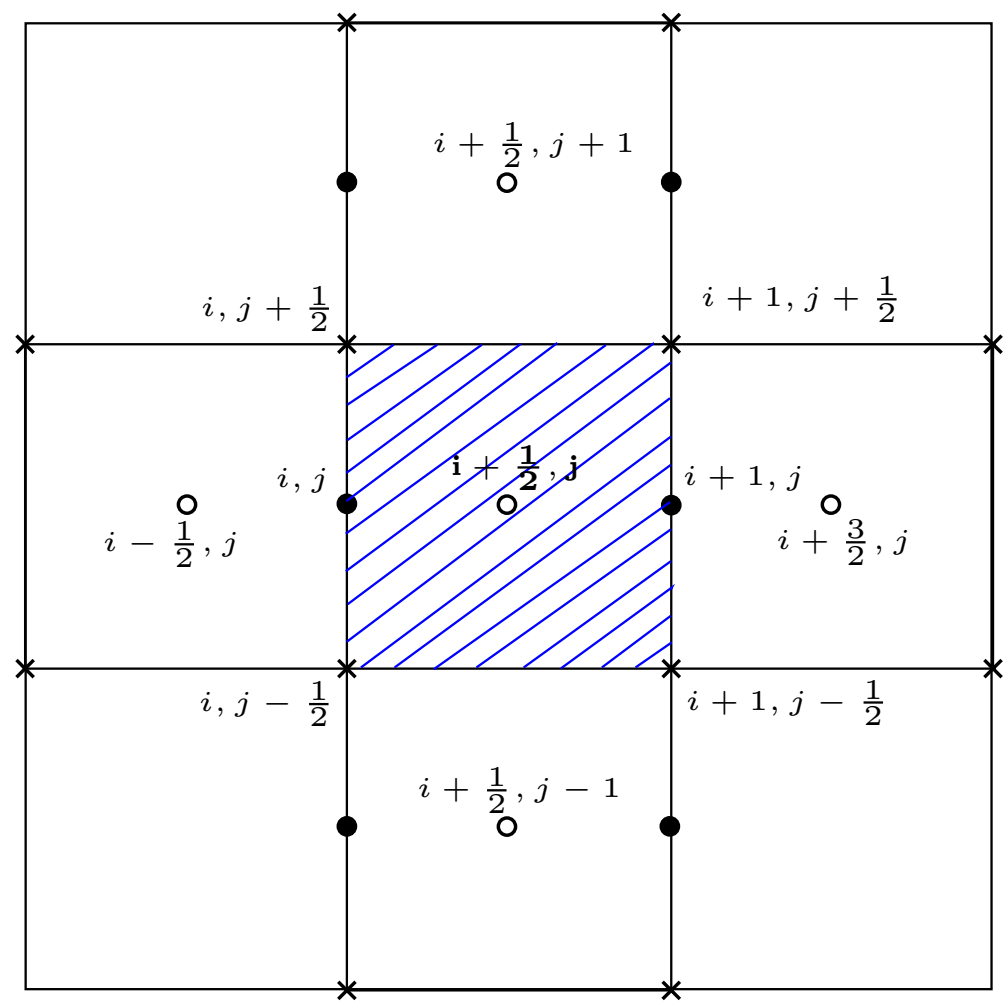

Figure 3.1: control volume for u, $E_{x}$ 


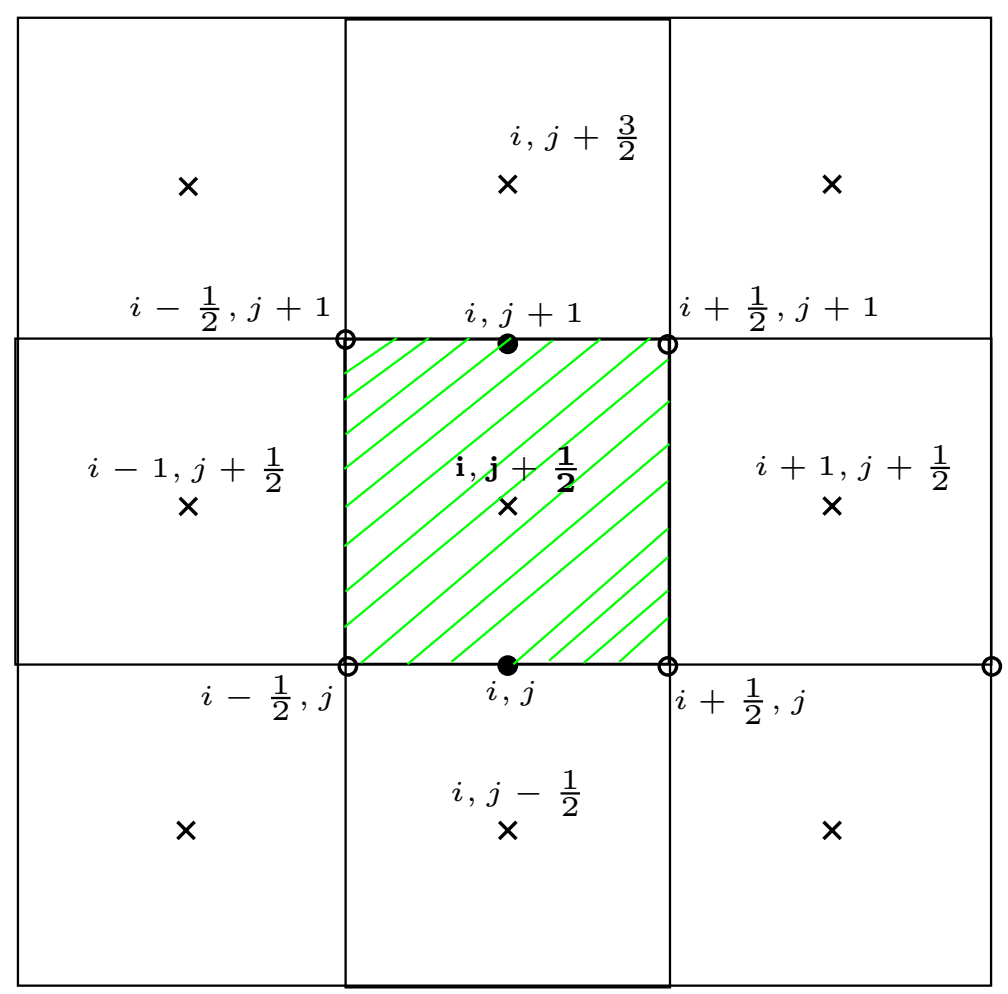

Figure 3.2: control volume for v, $E_{y}$

\subsubsection{Interface tracking}

Since we consider a $2 \mathrm{D}$ problem, the interface is a curve and is discretized using 1D line elements with two nodes (marker points). The interface front points are maintained as linked lists, which makes it easier to insert a new point when two neighbouring interface points drift too far apart or remove new points when two neighbouring points come extremely close to each other. The interfaces are tracked in a Lagrangian manner. Since the interfaces advect with the local fluid velocity, the interface velocity is computed after solving the Navier-Stokes equation at each step as

$$
\mathbf{u}\left(\mathbf{x}^{\prime}\right)=\int_{S} \mathbf{u}(\mathbf{x}) \delta^{2}\left(\mathbf{x}-\mathbf{x}^{\prime}\right) d \mathbf{x}
$$




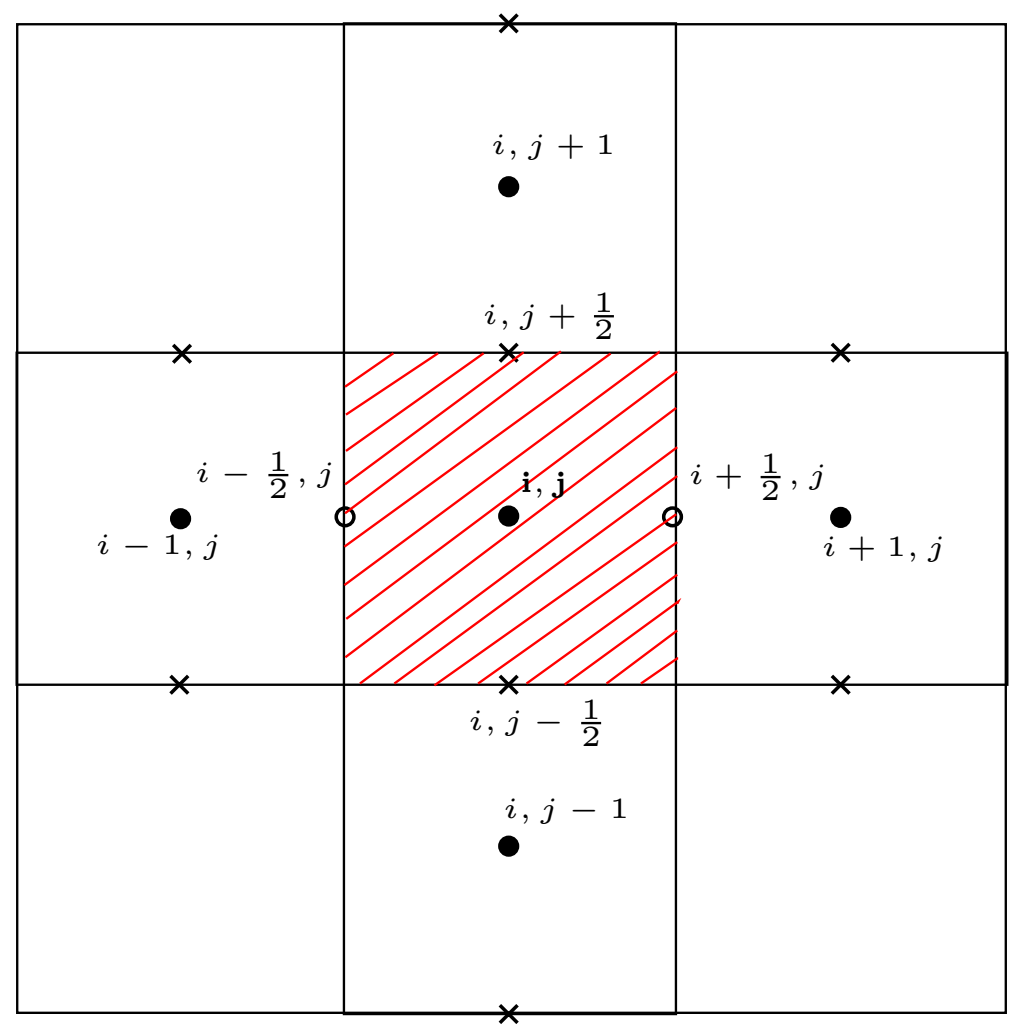

Figure 3.3: control volume for $p, \sigma, \mu, \epsilon, \rho$ and $\Phi$

where, $\mathrm{S}$ indicates the entire flow domain. The discrete delta function defined in (3.1.8) is used to represent $\delta$ in (3.1.11), resulting in a weighted mean interpolation of Eulerian fluid velocities which satisifies the continuity of velocity across the interfaces. The Lagrangian marker points are then advected as

$$
\frac{d \mathbf{x}^{\prime}}{d t}=\mathbf{u}\left(\mathbf{x}^{\prime}\right)
$$

First-order explicit Euler's method is used to numerically integrate the above equation in time.

$$
\mathbf{x}_{n+1}^{\prime}=\mathbf{x}_{n}^{\prime}+\Delta t \mathbf{u}\left(\mathbf{x}_{n}^{\prime}\right)
$$


where, $n, n+1$ denote the current and next time steps. As a result of using explicit Euler scheme, the overall numerical scheme is first order accurate in time. Secondorder accurate schemes have also been proposed [37], which will be implemented in the future works.

\subsubsection{Flow solver}

The initial interface positions, $\mathbf{x}^{(\mathbf{l})} \mathrm{s}$, are known. From the known interfacial coordinates, the gradient of the indicator function (Heaviside function) $(I(\mathbf{x}))$, denoted by $\mathbf{G}(\mathbf{x})$, is calculated as

$$
\mathbf{G}=\sum_{l} D\left(\mathbf{x}-\mathbf{x}^{(l)}\right) \mathbf{n}^{(l)} \Delta s^{(l)}
$$

where, $D\left(\mathbf{x}-\mathbf{x}^{(l)}\right)$ is defined in (3.1.8) and $\Delta s$ represents the length of the interface element. The indicator function is then obtained by solving the Poisson's equation (3.1.9), in the control volume of the figure 3.3, using the ADI method:

$$
A_{c} I_{i, j}+A_{r} I_{i+1, j}+A_{l} I_{i-1, j}+A_{t} I_{i, j+1}+A_{b} I_{i, j-1}+A_{0}=0
$$

where,

$$
\begin{aligned}
& A_{c}=\left(\frac{2}{\Delta x^{2}}+\frac{2}{\Delta y^{2}}\right) \\
& A_{r}=A_{l}=\frac{-1}{\Delta x^{2}}, A_{t}=A_{b}=\frac{-1}{\Delta y^{2}} \\
& A_{0}=\frac{\left(G_{x}\right)_{i+1, j}-\left(G_{x}\right)_{i-1, j}}{2 d x}+\frac{\left(G_{y}\right)_{i, j+1}-\left(G_{y}\right)_{i, j-1}}{2 d y}
\end{aligned}
$$


Here, $G_{x}$ and $G_{y}$ refer to the x- and y- components of $\mathbf{G}$ (a vector). Once the indicator function is known, the electrical conductivity field is obtained using (3.1.10) and the electric potential field is obtained by solving the Ohmic current equation. Referring to figure 3.3, integration of equation (3.1.3) in the control volume and rearrangement gives

$$
A_{c} \Phi_{i, j}+A_{r} \Phi_{i+1, j}+A_{l} \Phi_{i-1, j}+A_{t} \Phi_{i, j+1}+A_{b} \Phi_{i, j-1}=0
$$

where,

$$
\begin{aligned}
A_{c}= & -\left[\left(\frac{\sigma_{i+1, j}+\sigma_{i, j}}{2 \Delta x^{2}}\right)+\left(\frac{\sigma_{i, j}+\sigma_{i-1, j}}{2 \Delta x^{2}}\right)\right] \\
& -\left[\left(\frac{\sigma_{i, j+1}+\sigma_{i, j}}{2 \Delta y^{2}}\right)+\left(\frac{\sigma_{i, j}+\sigma_{i, j-1}}{2 \Delta y^{2}}\right)\right] \\
A_{r}= & \frac{1}{\Delta x^{2}}\left(\frac{\sigma_{i+1, j}+\sigma_{i, j}}{2}\right) \\
A_{l}= & \frac{1}{\Delta x^{2}}\left(\frac{\sigma_{i, j}+\sigma_{i-1, j}}{2}\right) \\
A_{t}= & \frac{1}{\Delta y^{2}}\left(\frac{\sigma_{i, j+1}+\sigma_{i, j}}{2}\right) \\
A_{b}= & \frac{1}{\Delta y^{2}}\left(\frac{\sigma_{i, j}+\sigma_{i, j-1}}{2}\right)
\end{aligned}
$$

(3.1.17) is solved using the Iterative Alternating Direction Implicit (ADI) method. The dielectric permittivity $(\epsilon)$ field is calculated using:

$$
\epsilon=\epsilon_{2}+\left(\epsilon_{1}-\epsilon_{2}\right) I(\mathbf{x})
$$


where, $\epsilon_{2}$ and $\epsilon_{1}$ are the dielectric permittivities of the sandwiched (phenol) and sandwiching (aqueous buffer) fluids respectively. The charge density $\left(\rho_{E}\right)$ can be determined using Gauss's law as

$$
-\nabla \cdot(\epsilon \nabla \Phi)=\rho_{E}
$$

Integration of equation (3.1.20) using the control volume of figure (3.3) gives:

$$
\begin{aligned}
\int_{V_{i, j}} \nabla \cdot(-\epsilon \nabla \Phi) d V= & \int_{\partial V_{i, j}}(-\epsilon \nabla \Phi) d A \\
= & \frac{-1}{\Delta x^{2}}\left[\left(\frac{\epsilon_{i+1, j}+\epsilon_{i, j}}{2}\right)\left(\Phi_{i+1, j}-\Phi_{i, j}\right)\right] \\
& \frac{-1}{\Delta x^{2}}\left[-\left(\frac{\epsilon_{i, j}+\epsilon_{i-1, j}}{2}\right)\left(\Phi_{i, j}-\Phi_{i-1, j}\right)\right] \\
& \frac{-1}{\Delta y^{2}}\left[\left(\frac{\epsilon_{i, j+1}+\epsilon_{i, j}}{2}\right)\left(\Phi_{i, j+1}-\Phi_{i, j}\right)\right] \\
& \frac{-1}{\Delta y^{2}}\left[-\left(\frac{\epsilon_{i, j}+\epsilon_{i, j-1}}{2}\right)\left(\Phi_{i, j}-\Phi_{i, j-1}\right)\right] \\
= & \left(\rho_{E}\right)_{i, j}
\end{aligned}
$$

Having obtained the electric potential and charge density fields, the next step is to solve the Navier-Stokes equation together with the continuity equation. Timeintegration of the Navier-Stokes equation is performed using the two-step projection method (Chorin [41]). The first step is called the "predictor step" in which an advection diffusion equation is solved, without including the pressure-gradient term. The expanded form of $\mathrm{x}$ - and $\mathrm{y}$ - momentum equations are 


$$
\begin{aligned}
\frac{\partial(\rho u)}{\partial t}+\frac{\partial\left(\rho u^{2}\right)}{\partial x}+\frac{\partial(\rho u v)}{\partial y}= & \frac{1}{R e}\left(2 \frac{\partial}{\partial x}\left(\mu \frac{\partial u}{\partial x}\right)+\frac{\partial}{\partial y}\left(\mu \frac{\partial v}{\partial x}\right)+\frac{\partial}{\partial y}\left(\mu \frac{\partial u}{\partial y}\right)\right) \\
& +\frac{1}{R e}\left(F_{e}\right)_{x} \\
& \quad(3.1 .22 \mathrm{a}) \\
\frac{\partial(\rho v)}{\partial t}+\frac{\partial(\rho u v)}{\partial x}+\frac{\partial\left(\rho v^{2}\right)}{\partial y}= & \frac{1}{R e}\left(\frac{\partial}{\partial x}\left(\mu \frac{\partial v}{\partial x}\right)+\frac{\partial}{\partial x}\left(\mu \frac{\partial u}{\partial y}\right)+2 \frac{\partial}{\partial y}\left(\mu \frac{\partial v}{\partial y}\right)\right) \\
& +\frac{1}{R e}\left(F_{e}\right)_{y}
\end{aligned}
$$

where, $\left(F_{e}\right)_{x}$ and $\left(F_{e}\right)_{y}$ are the x- and y-components of the electric body force $\mathbf{F}_{e}$. The density and viscosity fields for the next time step $\rho^{n+1}, \mu^{n+1}$ are determined as:

$$
\begin{gathered}
\rho=\rho_{2}+\left(\rho_{1}-\rho_{2}\right) I(\mathbf{x}) \\
\mu=\mu_{2}+\left(\mu_{1}-\mu_{2}\right) I(\mathbf{x})
\end{gathered}
$$

where, $\rho_{2}, \mu_{2}$ are the density and viscosity of the organic fluid, while $\rho_{1}, \mu_{1}$ are the density and viscosity of the aqueous fluid. In the time-integration, the non-linear terms are treated explicitly using the second-order Adams-Bashforth scheme and the diffusion, electric body force terms are treated semi-implicitly using the second-order Crank-Nicholson scheme. The discretized advection-diffusion equation is then solved using the Iterative Alternating Direction Implicit (ADI) method. For the sake of convenience, in the discretized form of the x-momentum 
equation (3.1.22a), we denote the nonlinear terms as INX1 and INX2, diffusion terms as VIX1, VIX2 and VIX3, electric body force terms as ELX1 and ELX2 respectively. Similar notations are employed for the discretized form of $\mathrm{y}$-momentum equation. The $\mathrm{x}$-momentum and $\mathrm{y}$-momentum equations on discretization yield:

$$
\begin{aligned}
\frac{(\rho u)^{*}-(\rho u)^{n}}{\Delta t} & =-\frac{3}{2}[\mathrm{INX} 1+\mathrm{INX} 2]^{n}+\frac{1}{2}[\mathrm{INX} 1+\mathrm{INX} 2]^{n-1} \\
& +\frac{1}{2 R e}[\mathrm{VIX} 1+\mathrm{VIX} 2+\mathrm{VIX} 3]^{*}+\frac{1}{2 R e}[\mathrm{VIX} 1+\mathrm{VIX} 2+\mathrm{VIX} 3]^{n} \\
& +\frac{1}{2 R e}[\mathrm{ELX} 1+\mathrm{ELX} 2]^{*}+\frac{1}{2 R e}[\mathrm{ELX} 1+\mathrm{ELX} 2]^{n}, \quad(3.1 .25 \mathrm{a}) \\
\frac{(\rho v)^{*}-(\rho v)^{n}}{\Delta t} & =-\frac{3}{2}[\mathrm{INY} 1+\mathrm{INY} 2]^{n}+\frac{1}{2}[\mathrm{INY} 1+\mathrm{INY} 2]^{n-1} \\
& +\frac{1}{2 R e}[\mathrm{VIY} 1+\mathrm{VIY} 2+\mathrm{VIY} 3]^{*}+\frac{1}{2 R e}[\mathrm{VIY} 1+\mathrm{VIY} 2+\mathrm{VIY} 3]^{n} \\
& +\frac{1}{2 R e}[\mathrm{ELY} 1+\mathrm{ELY} 2]^{*}+\frac{1}{2 R e}[\mathrm{ELY} 1+\mathrm{ELY} 2]^{n},
\end{aligned}
$$

where $\left[u^{*}, v^{*}\right]$ are velocities at an intermediate time between " $\mathrm{n}$ " and " $\mathrm{n}+1$ ", which do not satisfy the continuity equation and hence, aren't divergence free. For the spatial discretization, we employ the second-order central differencing. Considering the control volume for u-velocity as illustrated in figure 3.1, the spatial discretization scheme for various terms in equation (3.1.25a) yields:

$$
\mathrm{INX} 1=\frac{1}{\Delta x}\left[\rho_{i+1, j}\left(\frac{u_{i+\frac{3}{2}, j}+u_{i+\frac{1}{2}, j}}{2}\right)^{2}-\rho_{i, j}\left(\frac{u_{i+\frac{1}{2}, j}+u_{i-\frac{1}{2}, j}}{2}\right)^{2}\right]
$$




$$
\begin{aligned}
& \operatorname{INX} 2=\frac{1}{\Delta y}\left(\frac{\rho_{i, j+1}+\rho_{i, j}+\rho_{i+1, j+1}+\rho_{i+1, j}}{4}\right)\left(\frac{v_{i+1, j+\frac{1}{2}}+v_{i, j+\frac{1}{2}}}{2}\right) \\
& \left(\frac{u_{i+\frac{1}{2}, j+1}+u_{i+\frac{1}{2}, j}}{2}\right) \\
& \frac{-1}{\Delta y}\left(\frac{\rho_{i, j}+\rho_{i, j-1}+\rho_{i+1, j}+\rho_{i+1, j-1}}{4}\right)\left(\frac{v_{i+1, j-\frac{1}{2}}+v_{i, j-\frac{1}{2}}}{2}\right) \\
& \left(\frac{u_{i+\frac{1}{2}, j}+u_{i+\frac{1}{2}, j-1}}{2}\right) \\
& \mathrm{VIX} 1=\frac{1}{\Delta x^{2}}\left[\mu_{i+1, j}\left(u_{i+\frac{3}{2}, j}-u_{i+\frac{1}{2}, j}\right)-\mu_{i, j}\left(u_{i+\frac{1}{2}, j}-u_{i-\frac{1}{2}, j}\right)\right] \\
& \operatorname{VIX} 2=\frac{1}{\Delta y^{2}}\left[\left(\frac{\mu_{i, j+1}+\mu_{i, j}+\mu_{i+1, j+1}+\mu_{i+1, j}}{4}\right)\left(u_{i+\frac{1}{2}, j+1}-u_{i+\frac{1}{2}, j}\right)\right] \\
& -\frac{1}{\Delta y^{2}}\left[\left(\frac{\mu_{i, j}+\mu_{i, j-1}+\mu_{i+1, j}+\mu_{i+1, j-1}}{4}\right)\left(u_{i+\frac{1}{2}, j}-u_{i+\frac{1}{2}, j-1}\right)\right] \\
& \mathrm{VIX} 3=\frac{1}{\Delta x \Delta y}\left[\left(\frac{\mu_{i, j+1}+\mu_{i, j}+\mu_{i+1, j+1}+\mu_{i+1, j}}{4}\right)\left(v_{i+1, j+\frac{1}{2}}-v_{i, j+\frac{1}{2}}\right)\right] \\
& -\frac{1}{\Delta x \Delta y}\left[\left(\frac{\mu_{i, j}+\mu_{i, j-1}+\mu_{i+1, j}+\mu_{i+1, j-1}}{4}\right)\left(v_{i+1, j-\frac{1}{2}}-v_{i, j-\frac{1}{2}}\right)\right] \\
& \mathrm{ELX} 1=\left[\frac{\left(\rho_{E}\right)_{i+1, j}+\left(\rho_{E}\right)_{i, j}}{2}\right]\left(E_{x}\right)_{i+\frac{1}{2}, j} \\
& \mathrm{ELX} 2=\left[\frac{1}{2}\left(\left(E_{x}^{2}\right)_{i+\frac{1}{2}, j}+\left(E_{y}^{2}\right)_{i+\frac{1}{2}, j}\right)\left(\epsilon_{i, j}-\epsilon_{i+1, j}\right)\right] \text {, }
\end{aligned}
$$

Similarly, considering the control volume for $\mathrm{v}$ as illustrated in figure 3.2 , the spatial discretization scheme for various terms in equation (3.1.25b) gives:

$$
\begin{aligned}
\mathrm{INY} 1= & \frac{1}{\Delta x}\left(\frac{\rho_{i, j+1}+\rho_{i, j}+\rho_{i+1, j+1}+\rho_{i+1, j}}{4}\right)\left(\frac{u_{i+\frac{1}{2}, j+1}+u_{i+\frac{1}{2}, j}}{2}\right) \\
& \left(\frac{v_{i+1, j+\frac{1}{2}}+v_{i, j+\frac{1}{2}}}{2}\right) \\
& \frac{-1}{\Delta x}\left(\frac{\rho_{i, j}+\rho_{i, j+1}+\rho_{i-1, j}+\rho_{i-1, j+1}}{4}\right)\left(\frac{u_{i-\frac{1}{2}, j+1}+u_{i-\frac{1}{2}, j}}{2}\right) \\
& \left(\frac{v_{i, j+\frac{1}{2}}+v_{i-1, j+\frac{1}{2}}}{2}\right) \\
\mathrm{INY2} \mathrm{=} & \frac{1}{\Delta y}\left[\rho_{i, j+1}\left(\frac{v_{i, j+\frac{3}{2}}+v_{i, j+\frac{1}{2}}}{2}\right)^{2}-\rho_{i, j}\left(\frac{v_{i, j+\frac{1}{2}}+v_{i, j-\frac{1}{2}}}{2}\right)^{2}\right]
\end{aligned}
$$




$$
\begin{aligned}
\mathrm{VIY} 1 & =\frac{1}{\Delta y^{2}}\left[\mu_{i, j+1}\left(v_{i, j+\frac{3}{2}}-v_{i, j+\frac{1}{2}}\right)-\mu_{i, j}\left(v_{i, j+\frac{1}{2}}-v_{i, j-\frac{1}{2}}\right)\right] \\
\mathrm{VIY} 2 & =\frac{1}{\Delta x^{2}}\left[\left(\frac{\mu_{i, j+1}+\mu_{i, j}+\mu_{i+1, j+1}+\mu_{i+1, j}}{4}\right)\left(v_{i+1, j+\frac{1}{2}}-v_{i, j+\frac{1}{2}}\right)\right] \\
& -\frac{1}{\Delta x^{2}}\left[\left(\frac{\mu_{i, j}+\mu_{i, j+1}+\mu_{i-1, j}+\mu_{i-1, j+1}}{4}\right)\left(v_{i, j+\frac{1}{2}}-v_{i-1, j+\frac{1}{2}}\right)\right] \\
\mathrm{VIY} 3 & =\frac{1}{\Delta x \Delta y}\left[\left(\frac{\mu_{i, j+1}+\mu_{i, j}+\mu_{i+1, j+1}+\mu_{i+1, j}}{4}\right)\left(u_{i+\frac{1}{2}, j+1}-u_{i+\frac{1}{2}, j}\right)\right] \\
& -\frac{1}{\Delta x \Delta y}\left[\left(\frac{\mu_{i, j}+\mu_{i, j+1}+\mu_{i-1, j}+\mu_{i-1, j+1}}{4}\right)\left(u_{i-\frac{1}{2}, j+1}-u_{i-\frac{1}{2}, j}\right)\right] \\
\mathrm{ELY} 1 & =\left[\frac{\left(\rho_{E}\right)_{i, j}+\left(\rho_{E}\right)_{i, j+1}}{2}\right]\left(E_{y}\right)_{i, j+\frac{1}{2}} \\
\mathrm{ELY} 2 & =\left[\frac{1}{2}\left(\left(E_{x}^{2}\right)_{i, j+\frac{1}{2}}+\left(E_{y}^{2}\right)_{i, j+\frac{1}{2}}\right)\left(\epsilon_{i, j}-\epsilon_{i, j+1}\right)\right]
\end{aligned}
$$

Substituting the terms in (3.1.26) into the x-momentum equation (3.1.25a) and rearranging, we get,

$$
A_{c} u_{i+\frac{1}{2}, j}^{*}+A_{r} u_{i+\frac{3}{2}, j}^{*}+A_{l} u_{i-\frac{1}{2}, j}^{*}+A_{t} u_{i+\frac{1}{2}, j+1}^{*}+A_{b} u_{i+\frac{1}{2}, j-1}^{*}+A_{0}=0
$$

where, the coefficients are given by,

$$
\begin{aligned}
A_{c}= & \left(\frac{\rho_{i, j}^{*}+\rho_{i+1, j}^{*}}{2}\right)+\frac{\Delta t}{\operatorname{Re} \Delta x^{2}} \mu_{i, j}^{*}+\frac{\Delta t}{\operatorname{Re} \Delta x^{2}} \mu_{i+1, j}^{*} \\
& +\frac{\Delta t}{2 R e \Delta y^{2}}\left(\frac{\mu_{i, j+1}^{*}+\mu_{i, j}^{*}+\mu_{i+1, j+1}^{*}+\mu_{i+1, j}^{*}}{4}\right) \\
& +\frac{\Delta t}{2 \operatorname{Re} \Delta y^{2}}\left(\frac{\mu_{i, j}^{*}+\mu_{i, j-1}^{*}+\mu_{i+1, j}^{*}+\mu_{i+1, j-1}^{*}}{4}\right) \\
A_{r}= & -\frac{\Delta t}{\operatorname{Re} \Delta x^{2}} \mu_{i+1, j}^{*} \\
A_{l}= & -\frac{\Delta t}{\operatorname{Re} \Delta x^{2}} \mu_{i, j}^{*} \\
A_{t}= & -\frac{\Delta t}{2 \operatorname{Re} \Delta y^{2}}\left(\frac{\mu_{i, j+1}^{*}+\mu_{i, j}^{*}+\mu_{i+1, j+1}^{*}+\mu_{i+1, j}^{*}}{4}\right)
\end{aligned}
$$




$$
\begin{aligned}
A_{b}= & -\frac{\Delta t}{2 R e \Delta y^{2}}\left(\frac{\mu_{i, j}^{*}+\mu_{i, j-1}^{*}+\mu_{i+1, j}^{*}+\mu_{i+1, j-1}^{*}}{4}\right) \\
A_{0}= & -\frac{\Delta t}{2 R e \Delta x \Delta y}\left(\frac{\mu_{i, j+1}^{*}+\mu_{i, j}^{*}+\mu_{i+1, j+1}^{*}+\mu_{i+1, j}^{*}}{4}\right)\left(v_{i+1, j+\frac{1}{2}}^{*}-v_{i, j+\frac{1}{2}}^{*}\right) \\
& +\frac{\Delta t}{2 R e \Delta x \Delta y}\left(\frac{\mu_{i, j}^{*}+\mu_{i, j-1}^{*}+\mu_{i+1, j}^{*}+\mu_{i+1, j-1}^{*}}{4}\right)\left(v_{i+1, j-\frac{1}{2}}^{*}-v_{i, j-\frac{1}{2}}^{*}\right) \\
& -\left(\frac{\rho_{i, j}^{n}+\rho_{i+1, j}^{n}}{2}\right) u_{i+\frac{1}{2}, j}^{n}+\frac{3}{2} \Delta t[I N X 1]^{n}-\frac{1}{2} \Delta t[I N X 1]^{n-1} \\
& +\frac{3}{2} \Delta t[I N X 2]^{n}-\frac{1}{2} \Delta t[I N X 2]^{n-1}-\frac{\Delta t}{R e}[V I X 1]^{n}-\frac{\Delta t}{2 R e}[V I X 2]^{n} \\
& -\frac{\Delta t}{2 R e}[V I X 3]^{n}-\frac{\Delta t}{2 R e}[E L X 1]^{*}-\frac{\Delta t}{2 R e}[E L X 1]^{n}-\frac{\Delta t}{2 R e}[E L X 2]^{*} \\
& -\frac{\Delta t}{2 R e}[E L X 2]^{n},
\end{aligned}
$$

Similarly, substituting the terms (3.1.27) into the y-momentum equation (3.1.25b) and rearranging, we obtain,

$$
A_{c} v_{i, j+\frac{1}{2}}^{*}+A_{r} v_{i+1, j+\frac{1}{2}}^{*}+A_{l} v_{i-1, j+\frac{1}{2}}^{*}+A_{t} v_{i, j+\frac{3}{2}}^{*}+A_{b} v_{i, j-\frac{1}{2}}^{*}+A_{0}=0
$$

where, the coefficients are given by,

$$
\begin{aligned}
A_{c}= & \left(\frac{\rho_{i, j+1}^{*}+\rho_{i, j}^{*}}{2}\right)+\frac{\Delta t}{R e \Delta y^{2}} \mu_{i, j}^{*}+\frac{\Delta t}{R e \Delta y^{2}} \mu_{i, j+1}^{*} \\
& +\frac{\Delta t}{2 \operatorname{Re} \Delta x^{2}}\left(\frac{\mu_{i, j+1}^{*}+\mu_{i, j}^{*}+\mu_{i+1, j+1}^{*}+\mu_{i+1, j}^{*}}{4}\right) \\
& +\frac{\Delta t}{2 \operatorname{Re} \Delta x^{2}}\left(\frac{\mu_{i, j}^{*}+\mu_{i, j+1}^{*}+\mu_{i-1, j}^{*}+\mu_{i-1, j+1}^{*}}{4}\right) \\
A_{r}= & -\frac{\Delta t}{2 \operatorname{Re} \Delta x^{2}}\left(\frac{\mu_{i, j+1}^{*}+\mu_{i, j}^{*}+\mu_{i+1, j+1}^{*}+\mu_{i+1, j}^{*}}{4}\right) \\
A_{l}= & -\frac{\Delta t}{2 \operatorname{Re} \Delta x^{2}}\left(\frac{\mu_{i, j}^{*}+\mu_{i, j+1}^{*}+\mu_{i-1, j}^{*}+\mu_{i-1, j+1}^{*}}{4}\right) \\
A_{t}= & -\frac{\Delta t}{\operatorname{Re} \Delta y^{2}} \mu_{i, j+1}^{*}
\end{aligned}
$$




$$
\begin{aligned}
A_{b}= & -\frac{\Delta t}{R e \Delta y^{2}} \mu_{i, j}^{*} \\
A_{0}= & -\frac{\Delta t}{2 R e \Delta x \Delta y}\left(\frac{\mu_{i, j+1}^{*}+\mu_{i, j}^{*}+\mu_{i+1, j+1}^{*}+\mu_{i+1, j}^{*}}{4}\right)\left(u_{i+\frac{1}{2}, j+1}^{*}-u_{i+\frac{1}{2}, j}^{*}\right) \\
& +\frac{\Delta t}{2 R e \Delta x \Delta y}\left(\frac{\mu_{i, j}^{*}+\mu_{i, j+1}^{*}+\mu_{i-1, j}^{*}+\mu_{i-1, j+1}^{*}}{4}\right)\left(u_{i-\frac{1}{2}, j+1}^{*}-u_{i-\frac{1}{2}, j}^{*}\right) \\
& -\left(\frac{\rho_{i, j}^{n}+\rho_{i, j+1}^{n}}{2}\right) v_{i, j+\frac{1}{2}}^{n}+\frac{3}{2} \Delta t[I N Y 1]^{n}-\frac{1}{2} \Delta t[I N Y 1]^{n-1} \\
& +\frac{3}{2} \Delta t[I N Y 2]^{n}-\frac{1}{2} \Delta t[I N Y 2]^{n-1}-\frac{\Delta t}{R e}[V I Y 1]^{n}-\frac{\Delta t}{2 R e}[V I Y 2]^{n} \\
& -\frac{\Delta t}{2 R e}[V I Y 3]^{n}-\frac{\Delta t}{2 R e}[E L Y 1]^{*}-\frac{\Delta t}{2 R e}[E L Y 1]-\frac{\Delta t}{2 R e}[E L Y 2]^{*} \\
& -\frac{\Delta t}{2 R e}[E L Y 2]^{n},
\end{aligned}
$$

Equations (3.1.28) and (3.1.30) are solved using the iterative ADI method to obtain the velocities $u^{*}$ and $v^{*}$.

Next, we move to the second step of the projection method called the corrector step in which we include the pressure gradient term and solve for the velocity field at time step "n+1"

$$
\frac{\mathbf{u}^{n+1}-\mathbf{u}^{*}}{\Delta t}=-\nabla P
$$

or, by taking divergence,

$$
\frac{\nabla \cdot \mathbf{u}^{n+1}-\nabla \cdot \mathbf{u}^{*}}{\Delta t}=-\nabla^{2} P
$$

where, the pressure field is obtained by using the constraint of $\mathbf{u}^{n+1}$ to be divergencefree and hence,

$$
\nabla^{2} P^{n+1}=\frac{\rho\left(\nabla \cdot \mathbf{u}^{*}\right)}{\Delta t}
$$


Considering the control volume in figure 3.3, the Poisson's equation for pressure (3.1.33) can be discretized to obtain

$$
a P_{i, j}^{n+1}+b P_{i+1, j}^{n+1}+c P_{i-1, j}^{n+1}+d P_{i, j+1}^{n+1}+e P_{i, j-1}^{n+1}+f=0
$$

where, the coefficients are given by,

$$
\begin{aligned}
a & =\left[\frac{\Delta t}{\Delta x^{2} \rho_{i+\frac{1}{2}, j}^{n+1}}+\frac{\Delta t}{\Delta x^{2} \rho_{i-\frac{1}{2}, j}^{n+1}}+\frac{\Delta t}{\Delta y^{2} \rho_{i, j+\frac{1}{2}}^{n+1}}+\frac{\Delta t}{\Delta y^{2} \rho_{i, j-\frac{1}{2}}^{n+1}}\right] \\
b & =-\frac{\Delta t}{\Delta x^{2} \rho_{i+\frac{1}{2}, j}^{n+1}} \\
c & =-\frac{\Delta t}{\Delta x^{2} \rho_{i-\frac{1}{2}, j}^{n+1}} \\
d & =-\frac{\Delta t}{\Delta y^{2} \rho_{i, j+\frac{1}{2}}^{n+1}} \\
e & =-\frac{\Delta t}{\Delta y^{2} \rho_{i, j-\frac{1}{2}}^{n+1}} \\
f & =\frac{1}{\Delta x}\left[\left(u_{i+\frac{1}{2}, j}^{*}-u_{i-\frac{1}{2}, j}^{*}\right)\right]+\frac{1}{\Delta y}\left[\left(v_{i, j+\frac{1}{2}}^{*}-v_{i, j-\frac{1}{2}}^{*}\right)\right],
\end{aligned}
$$

In the above equation, "f" is known as the source term. The solution to the pressure Poisson equation should be such that the source term is close to machine zero for the continuity equation to be satisfied. The pressure Poisson equation can be solved with the iterative alternating-direction-implicit (ADI) technique. 


\subsection{Validation}

\subsubsection{Step size accuracy}

The front tracking algorithm implemented for the current study is first-order accurate in time step size $\Delta t$ and second-order accurate in spatial discretization size $\Delta x$. We verify the time step size accuracy for two different cases: (1) with electrical conductivity variation alone and (2) with electrical conductivity and density variations, using data collected from simulations with different time step sizes. It is worth mentioning that in the following sections, time step sizes $\Delta t$ and grid sizes $\Delta x$ are dimensionless. Time step sizes $\Delta t=5 \times 10^{-4}, 1 \times 10^{-3}, 1.25 \times$ $10^{-3}, 2 \times 10^{-3}, 2.5 \times 10^{-3}, 4 \times 10^{-3}, 5 \times 10^{-3}, 8 \times 10^{-3}, 0.01,0.02,0.025,0.05$ and 0.1 were considered. Since there is neither an analytical solution nor Direct Numerical Simulation data available for the nonlinear problem under consideration, we assume that the data corresponding to $\Delta t=5 \times 10^{-4}$ is the most accurate and hence use that as the baseline for the relative error norm calculation. The u-velocity data has been used for the relative error norm calculation in both the afore-mentioned cases. Figures 3.4(a) and (b) show the log-log plot of the relative error norm (2-norm) against the time-step size for cases (1) and (2) respectively. The slope of the log-log plot gives us the order of accuracy with respect to the time-step size and we obtain slope values of 0.8123 and 0.7528 for cases (1) and (2) respectively. This verifies that the algorithm is approximately first-order accurate in time. 


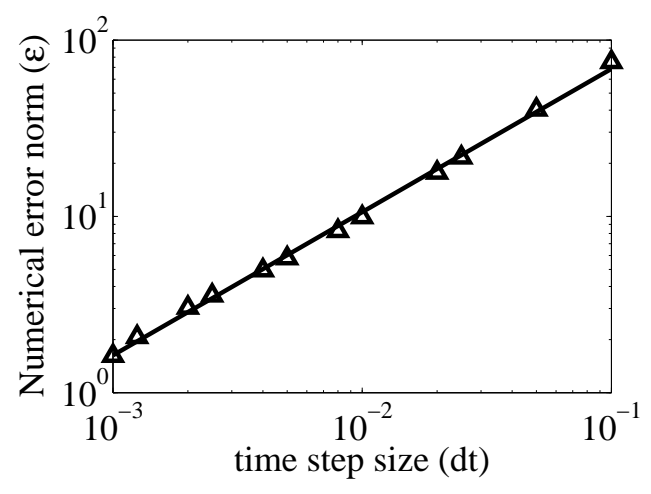

(a) $\sigma$ variation alone.

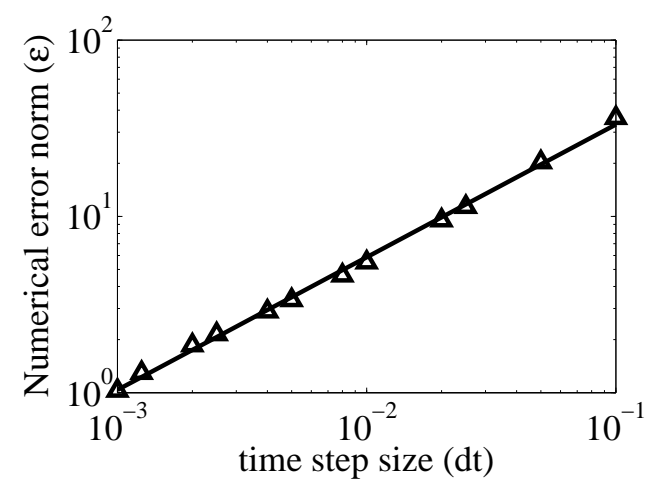

(b) $\sigma$ and $\rho$ variations.

Figure 3.4: Log-log plot for error norm variation with time-step size for (a) $\sigma$ variation alone and (b) $\sigma$ and $\rho$ variations. The slope values obtained are (a) 0.8123 and (b) 0.7528 respectively, meaning that the algorithm is approximately first-oder accurate.

\subsubsection{Growth rate calculation accuracy}

In our current work, we are primarily interested in comparing the growth rate values obtained from both the linear stability analysis and non-linear simulations. Hence, it would be worthwhile to verify the accuracy of the growth rate calculations using different time-step sizes. From the non-linear simulation data, we plot the maximum interfacial displacement (with respect to equilibrium configuration) against time. A least-squares (LS) fit is performed from which the slope is obtained, which provides us an estimate of the growth rate. The growth rate calculation from three different time-step values of $\Delta t=5 \times 10^{-4}, 1 \times 10^{-3}$ and $5 \times 10^{-3}$ for cases (1) and (2) are shown in Figures 3.5(a) and (b) respectively. For case (1) $\sigma$ variation alone, we obtain growth rate values of $1.87689 \times 10^{5} s^{-1}$, $1.87576 \times 10^{5} s^{-1}$ and $1.87409 \times 10^{5} s^{-1}$ for $\Delta t=5 \times 10^{-4}, 1 \times 10^{-3}$ and $5 \times 10^{-3}$ 


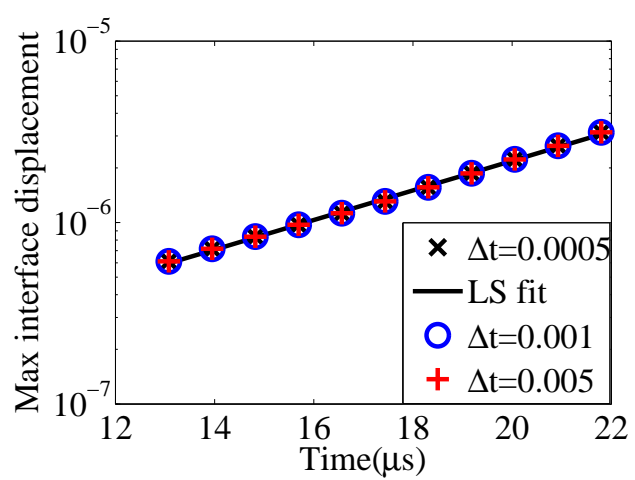

(a) $\sigma$ variation alone.

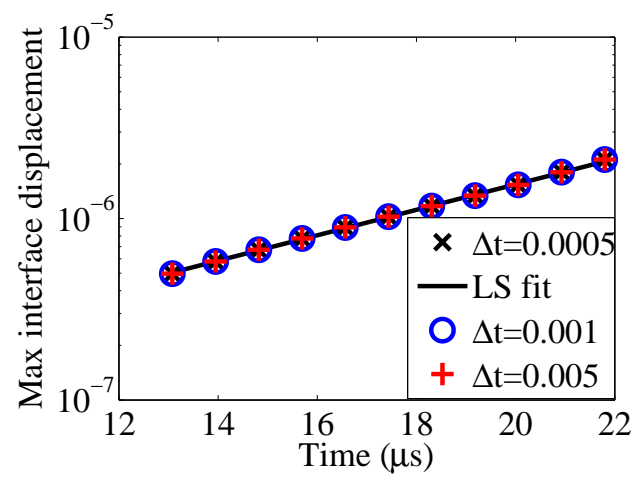

(b) $\sigma$ and $\rho$ variations.

Figure 3.5: Semi-log plot of maximum interface displacement with time for (a) $\sigma$ variation alone and (b) $\sigma$ and $\rho$ variations for three different time-step sizes. With respect to the baseline values obtained for $\Delta t=5 \times 10^{-4}$, the maximum error in the growth rate calculation for cases (a) and (b) are $0.15 \%$ and $0.11 \%$ respectively.

respectively. With respect to the value obtained for $\Delta t=5 \times 10^{-4}$, the maximum error in the growth rate calculation is $0.15 \%$. Similarly, for case (2) $\sigma$ and $\rho$ variations, we obtain growth rate values of $1.614 \times 10^{5} \mathrm{~s}^{-1}, 1.615 \times 10^{5} \mathrm{~s}^{-1}$ and $1.61579 \times 10^{5} s^{-1}$ for $\Delta t=5 \times 10^{-4}, 1 \times 10^{-3}$ and $5 \times 10^{-3}$ respectively. With respect to the baseline value from $\Delta t=5 \times 10^{-4}$, the maximum error in the growth rate calculation is $0.11 \%$. In the simulation results and growth rate results provided in subsequent sections, the time-step size employed in the numerical simulations is $5 \times 10^{-4}$, which has been observed to be highly accurate.

\subsubsection{Grid resolution validation}

Finally, we provide preliminary validation results with regard to grid resolution.

The growth rate results from the original and double grid resolution datasets are 


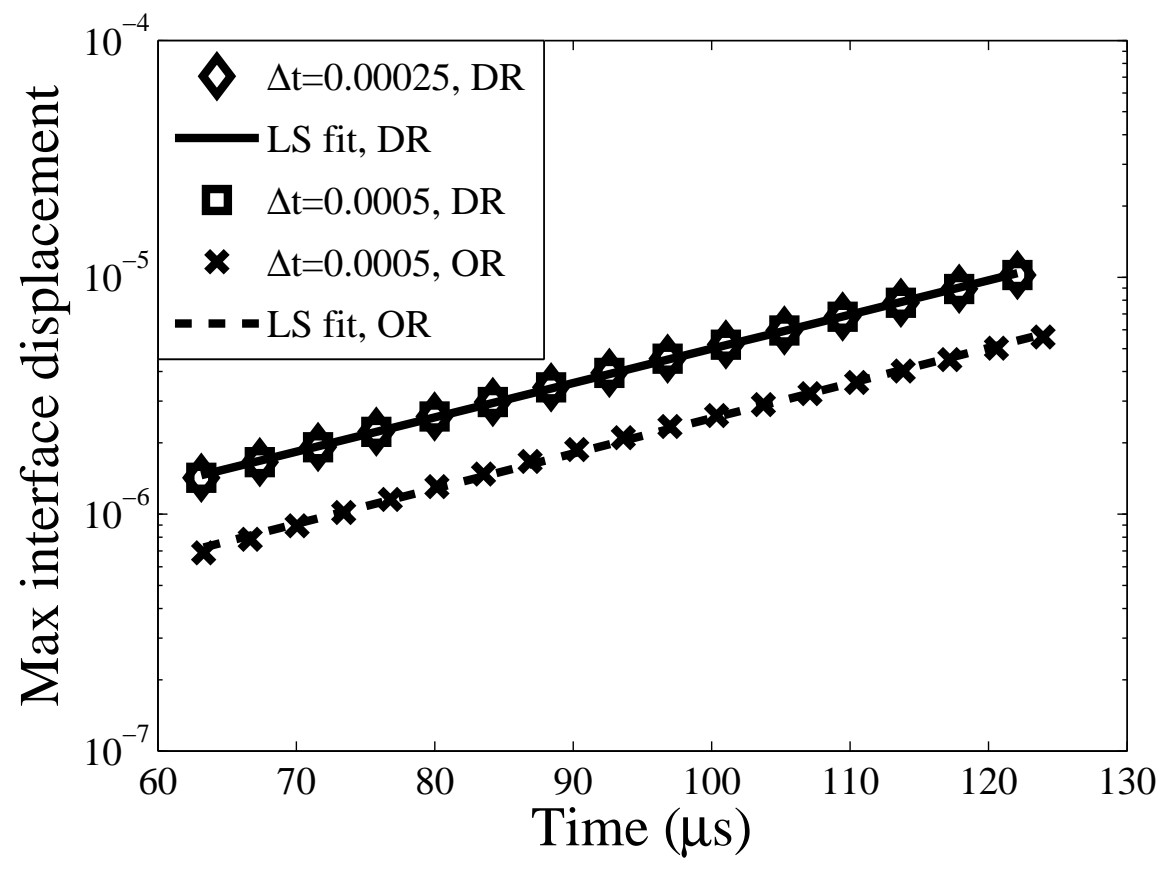

Figure 3.6: Growth rates extracted from nonlinear simulation for $k^{*}=10.0$, sausage mode for a conductivity ratio of 100 , density ratio of 0.752 , permittivity ratio of 10.974 , viscosity ratio of 0.284 . The extracted growth rate for double resolution (DR) case is $3.331 \times 10^{4} s^{-1}$, a $2.67 \%$ difference from the growth rate result obtained from the original resolution (OR) dataset captured in the same time interval.

compared and plotted in figure 3.2.3. It can be observed from the plot that data sets captured for the double resolution (mentioned as DR in the legend) case with two different time step sizes of $5 \times 10^{-4}$ and $2.5 \times 10^{-4}$ are in agreement with each other, thereby validating the time-step size accuracy. Such a validation is also necessitated by the fact that with increase in grid resolution and hence a decrease in $\Delta x$, there is a possibility of the Courant-Friedrichs-Levy (CFL) stability criterion violation. Such a violation was not observed with the grid resolution and time-step size combinations considered in the simulation. The slopes of the least-squares plot are $3.42 \times 10^{4} s^{-1}$ and $3.331 \times 10^{4} s^{-1}$ for the original resolution 
(mentioned as OR in the legend) and double grid resolution cases respectively, a relative error of around $3 \%$. Inspite of the excellent agreement with the growth rate results, the interfacial displacements between the two cases can be observed to be noticeably different. The reasons for this disagreement would be considered in a future study.

\subsubsection{Miscellaneous validations}

There are some additional validations performed which would not be covered in detail for the sake of brevity of the report. They are outlined briefly in the following subsections:

\section{Discretization of convective terms}

It is well understood that the convective terms are the major source of numerical issues in the solution of Navier-Stokes equations: (a) With the the centraldifferencing scheme mentioned earlier, unphysical oscillatory behaviour maybe observed in certain regions of flow where convection effects dominate viscous effects and (b) although the first-order upwind schemes sidestep difficulties mentioned in (a), numerical diffusion errors are introduced when the Reynolds number (Re) of the flow is increased. Hence, the numerical method needs to be validated with higher-order convective schemes to minimize both the oscillatory behaviour and artificial numerical diffusion. Some of these schemes were considered in numerical simulation of the hydraulic jump by Ferreira et al. [42]. In addition to the central-difference scheme (CD) implemented originally, higher-order schemes 
Quadratic Upstream Interpolation for Convective Kinematics [43] (QUICK) and Sharp and Monotonic Algorithm for Realistic Transport [44] (SMART) were also additionally considered and the results compared with the central-differencing scheme. The solution relative error norms were well within $1 \%$ meaning that the CD scheme was accurate enough.

\section{Effect of interface elements number}

Apart from discretizing the flow domain, we also discretize the top and bottom interfaces using 2D line elements. Tryggvason et al. [37] suggested that the number of interface elements to be around 4 or 5 per grid point. Our numerical scheme was tested with 3, 5, 10 and 20 elements per grid point and it was indeed found that 5 elements per grid point were providing accurate numerical results (less than $1 \%$ error with respect to the denser 10 and 20 element results). For our original resolution cases $(\Delta x=1 / 70)$, the number of interface elements used was 1100 while for the double resolution cases $(\Delta x=1 / 140), 2200$ elements were used.

\subsection{Results from nonlinear analysis}

In the following sections, we compare the growth rates from both the linear analysis and nonlinear simulation with each other. For this purpose, we initiated the nonlinear simulation with a single mode (kink or sausage), and capture the maximum interface deformation from the equilibrium position at various times. 
The resulting slope of the semi-log plot provides an estimate for the growth rate. We simulate the nonlinear two-phase instability mechanism for the experimental values of density ratio $\rho=0.75$, permittivity ratio $\epsilon=10.97$, viscosity ratio $\mu=0.28$ but utilize a conductivity ratio of $\sigma=100$ rather than the experimental value of 5405. As mentioned earlier, we also neglect surface tension effects in the current work. The parametric ratios used in the simulation results of the following sections are chosen in such a way that the variation between growth rate results obtained from linear stability analysis for (a) the parameters considered (i.e. $\sigma=100$ and $\gamma=1 \times 10^{-16} \mathrm{~N} / \mathrm{m}$ ) and (b) the experimental parameters (i.e. $\sigma=5405$ and $\gamma=1 \times 10^{-4} \mathrm{~N} / \mathrm{m}$ ) is less than $5 \%$. We briefly quantify using two examples here, the first in which we compare the growth rate results in the presence of conductivity variations alone, with all other property ratios equaling 1. The error resulting from considering $\sigma=100$ and $\gamma=1 \times 10^{-16} \mathrm{~N} / \mathrm{m}$ compared to the experimental values is around $3 \%$. In the second example, density, viscosity and permittivity variations are included. The growth rate result variation resulting from $\sigma=100$ and $\gamma=1 \times 10^{-16} \mathrm{~N} / \mathrm{m}$ with respect to the experimental values is around 2.3\%. A dimensionless wavenumber value of $k^{*}=10$ was used in the afore-mentioned results and the same value would also be used in our nonlinear simulations. Hence, the neglection of surface tention is justified for the parametric values considrered in our results. Nonetheless, the surface tension has been implemented in the linear code, and can be added straightforwardly in the nonlinear simulation following the Immersed Boundary Method outline provided in the previous chapter. For all cases the applied field strength is $\mathrm{E}=9 \mathrm{kV} / \mathrm{cm}$ 
and since the electric field is applied in the positive y-direction, positive charges accumulate on the bottom interface and negative charges accumulate on the top interface resulting in electric body forces on both the interfaces leading to interface deformation. The base flow field is set to be quiescent, unless mentioned otherwise.

\subsubsection{Electrical conductivity variation}

Firstly, we provide representative results for the case of electrical conductivity variation alone. The electrical conductivity ratio is 100 , while all the other property ratios are set to be equal to 1 . Figure 3.7 provides snapshots of the evolution of conductivity and charge density profiles respectively, at various time instants prior to the droplet formation. The initial condition in figure 3.7 is biased with a sausage-mode with a dimensionless wavenumber of $k^{*}=10$, and a dimensionless amplitude of $5 \times 10^{-3}$, both scaled with the channel half-width $(\mathrm{H}=75 \mu \mathrm{m})$. The electroviscous velocity scale, time scale and the Reynolds number are calculated to be $43.011 \mathrm{~m} / \mathrm{s}, 1.75 \mu \mathrm{s}$ and 3226 respectively. Note that regions of different conductivity also represent regions of different liquid, because the conductivity is uniform within a single phase. From the figures, it is clear that the interfacial deformations are out of phase with each other by $\pi$ meaning the profile evolution reflects the initial perturbation mode. From the linear analysis, the growth rate for the sausage-mode perturbation with a conductivity ratio $\sigma=100$, dimensionless wavenumber $k^{*}=10$ and electric field $\mathrm{E}=9 \times 10^{5} \mathrm{~V} / \mathrm{m}$ is determined to be 
$1.733 \times 10^{5} \mathrm{~s}^{-1}$. On the other hand, from the nonlinear simulation data collected

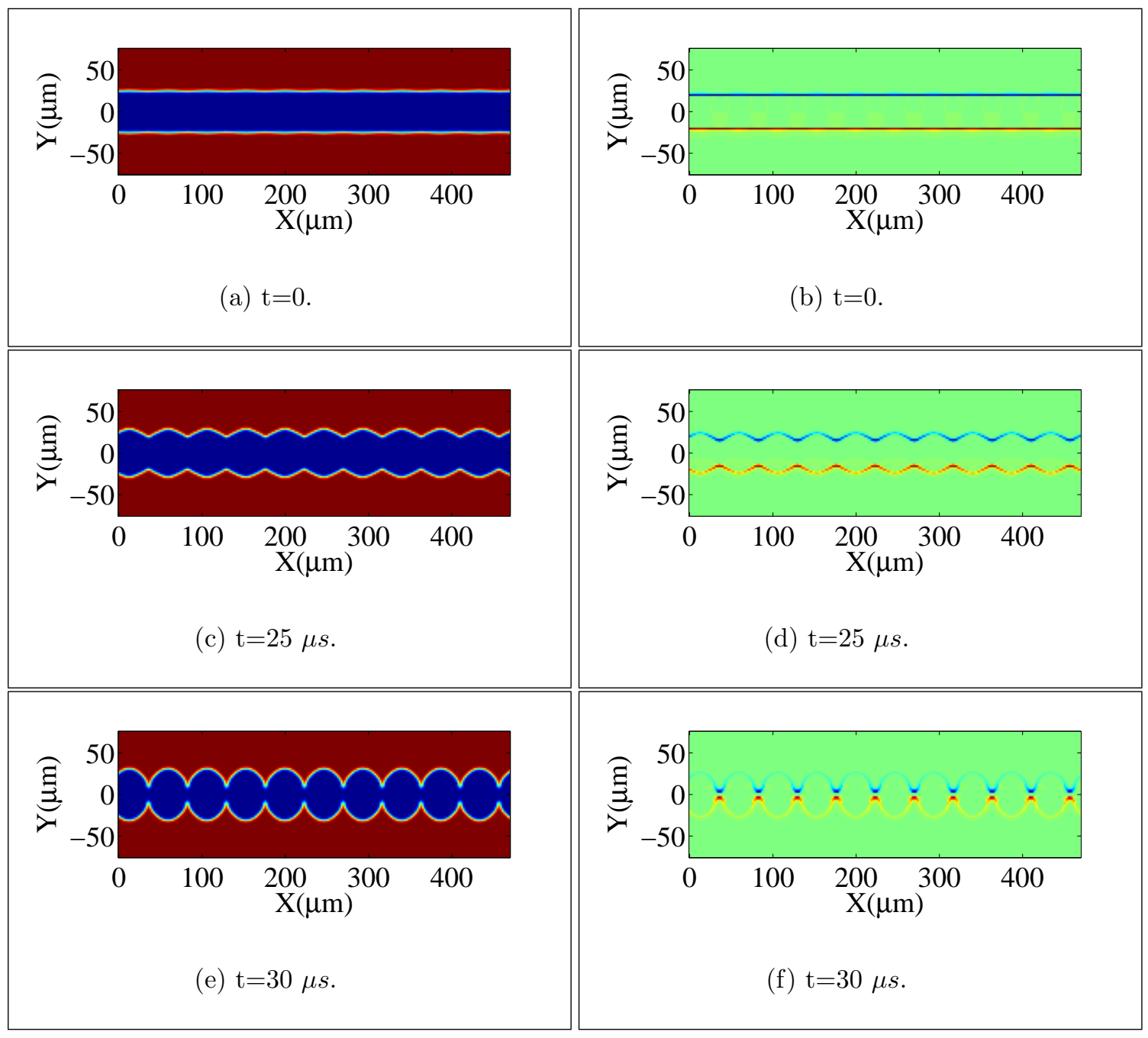

Figure 3.7: Electrical conductivity profile evolution for the case of conductivity ratio 100 and all other property ratios equal to 1 . The (a) initial profile, profiles at (c) $25 \mu \mathrm{s}$ and (e) $30 \mu \mathrm{s}$ for conductivity are shown. Red denotes the high conductivity regions (aqueous phase), and blue, the low conductivity region (phenol). (b), (d) and (f) depict the charge density snapshots at corresponding times.

in the linear regime, the slope of the Least-Squares fit for the semilog plot shown in figure 3.3 .1 is calculated to be $1.877 \times 10^{5} \mathrm{~s}^{-1}$. The relative error in growth rate calculation with respect to the linear analysis is $8.25 \%$, providing favourable agreement. The discrepancy arises possibly due to the nature of the front-tracking method used in nonlinear simulation. In this method, the interfacial forces are 


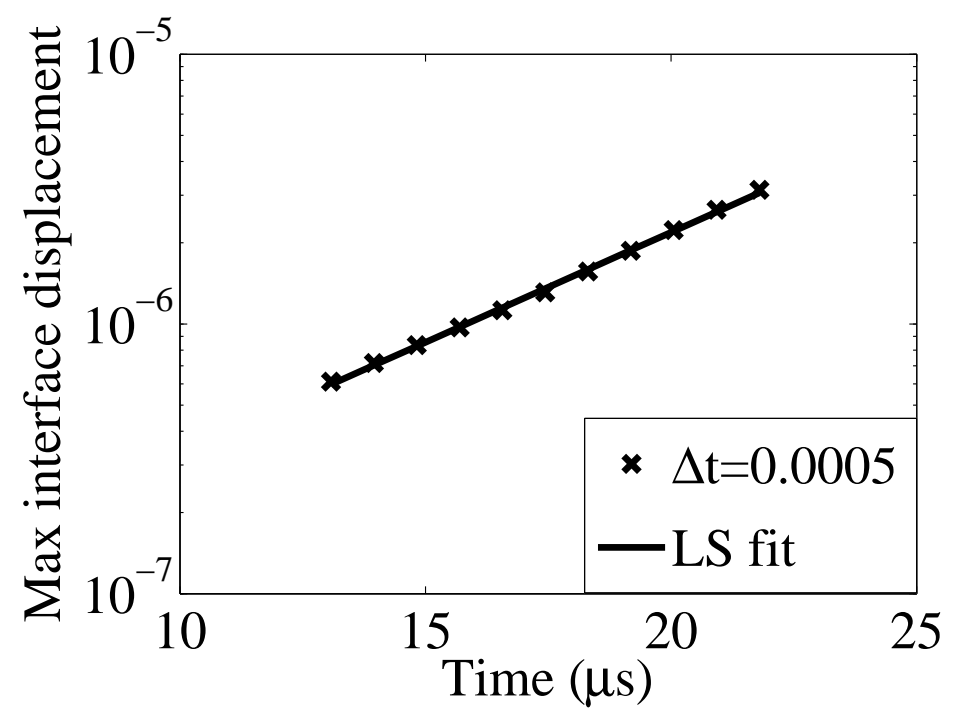

Figure 3.8: Growth rates extracted from nonlinear simulation for conductivity variation alone with $k^{*}=10.0$, sausage mode. The ' $x$ ' are from the simulation with a dimensionless time step size of $\Delta t=5 \times 10^{-4}$, and the line is from leastsquare (LS) fitting, from which the growth rates are calculated. The extracted growth rate is $1.877 \times 10^{5} \mathrm{~s}^{-1}$, an $8.25 \%$ difference from the linear analysis result.

spread over a finite width (typically a few grid points), whereas in the linear analysis the interfaces are considered sharp (with zero thickness).

\subsubsection{Electrical conductivity and density variations}

Next, we add density variation with the experimental density ratio value of 0.752 .

The organic fluid is slightly more dense compared to the aqueous fluid. An initial bias of a sausage-mode with a dimensionless wavenumber of $k^{*}=10$, and a dimensionless amplitude of $5 \times 10^{-3}$ is applied. The density profiles at two different time instants are shown in figure 3.9. The electroviscous velocity scale and time scale are the same as that for the conductivity variation case while the Reynolds number is 4290 which is higher because of introducing higher density 


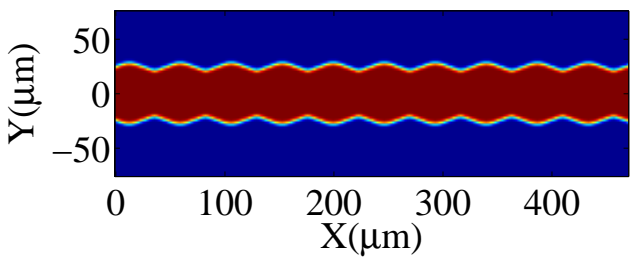

(a) $\mathrm{t}=26 \mu \mathrm{s}$.

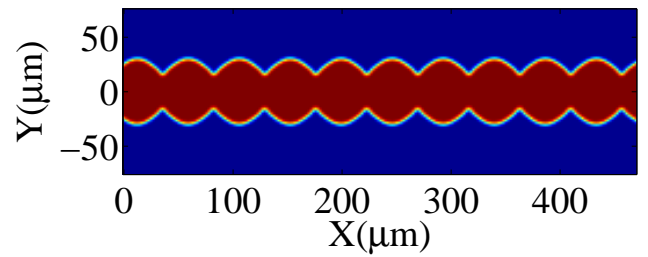

(b) $\mathrm{t}=30 \mu \mathrm{s}$.

Figure 3.9: Interface evolution for the case of conductivity ratio 100, density ratio 0.752 and all other property ratios equal to 1 . The profiles at (b) $26 \mu s$ and (c) $30 \mu s$ are shown. Red denotes the high density region (phenol), and blue, the low density regions (aqueous phase).

value for the organic fluid. Comparing the interface deformation profiles in the current case with the case of just conductivity variation alone, we do not observe any significant differences since the density ratio is close to 1 . Hence, we can predict that the growth rate results for these two cases would not show substantial difference and this indeed is found to be observed. The growth rate obtained from the linear analysis for this case is $1.619 \times 10^{5} \mathrm{~s}^{-1}$, while the slope of the nonlinear simulation data shown in figure 3.3.2 is $1.615 \times 10^{5} \mathrm{~s}^{-1}$. The relative error with respect to the linear analysis result is about $0.35 \%$, showing excellent agreement with the linear analysis result.

\subsubsection{Electrical conductivity, density and permittivity vari- ations}

The next level of complexity is the addition of dielectric permittivity variation. The experimental permittivity ratio value is 10.974 . The initial condition in this 


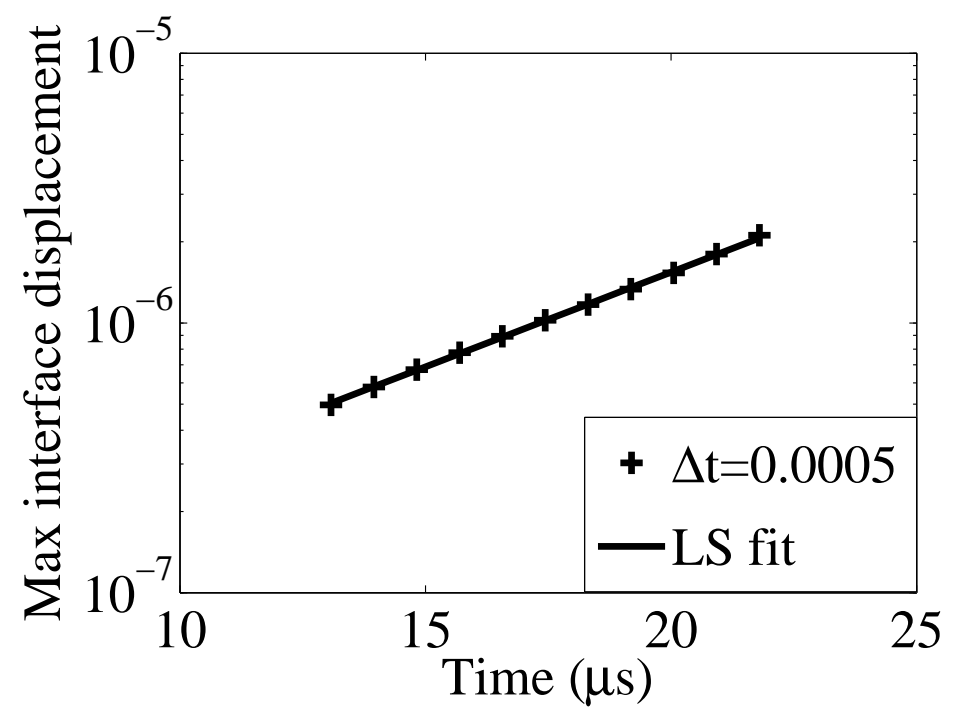

Figure 3.10: Growth rates extracted from nonlinear simulation with $k^{*}=10.0$, sausage mode for a conductivity ratio 100 and density ratio 0.752 . The ' + ' are from the simulation with a dimensionless time step size of $\Delta t=5 \times 10^{-4}$. The extracted growth rate is $1.615 \times 10^{5} \mathrm{~s}^{-1}$, a $0.35 \%$ difference from the linear analysis result.

case is taken to be a kink-mode disturbance with a dimensionless wavenumber of $k^{*}=10$, and a dimensionless amplitude of $5 \times 10^{-3}$. The permittivity profile and charge density evolution snapshots at two different instants of time are provided in figure 3.11. The electroviscous velocity scale for this case is calculated to be $3.92 \mathrm{~m} / \mathrm{s}$, time scale being $19.13 \mu \mathrm{s}$ and the Reynolds number is 391. We can predict from velocity scale that the growth rate value would be significantly lower than the previous cases. A similar conclusion can be arrived at by comparing the electroviscous time scales as well and this is substantiated by the calculated growth rate values. The growth rate from linear analysis for the kink-mode disturbance is obtained as $4.005 \times 10^{4} s^{-1}$. From the nonlinear simulation data plotted in figure 3.3.3, the slope of the least-squares fit is calculated to be $4.602 \times 10^{4} s^{-1}$, 


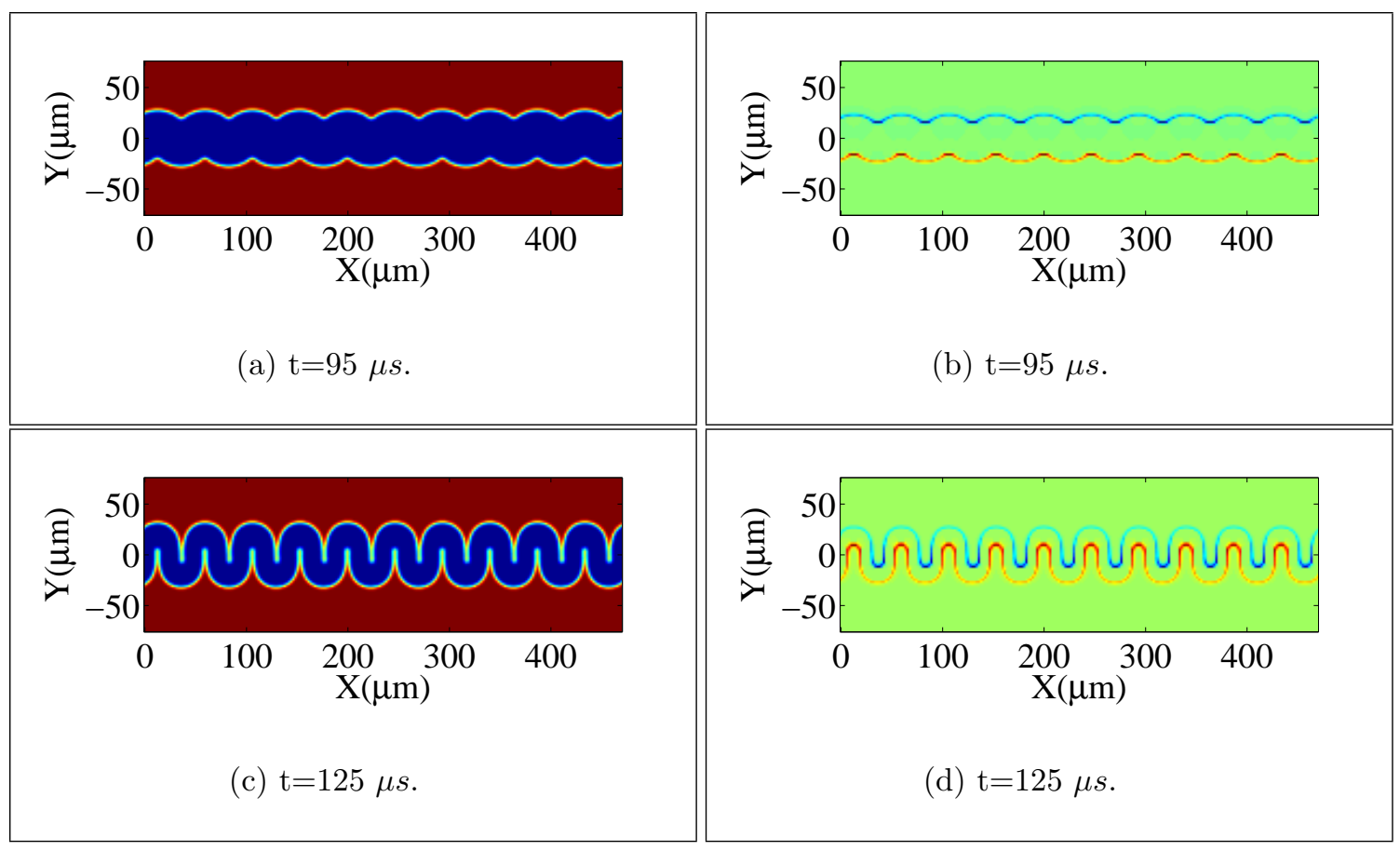

Figure 3.11: Interface evolution for the case of conductivity ratio 100, density ratio 0.752 , permittivity ratio 10.974 and viscosity ratio 1 . The profiles at (a) 95 $\mu s$ and (c) $125 \mu s$ for permittivity are shown. Red denotes the high permittivity regions (aqueous phase), and blue, the low permittivity region (phenol). The corresponding charge density snapshots are shown in (b) and (d) respectively.

a relative error of $14.9 \%$ compared to the linear analysis result. The magnitude of the relative error for the kink mode is observed to be higher than that for sausage mode (simulation results not shown here for brevity) perturbation. The exact reason for this difference is yet to be determined and will be analyzed in a future investigation.

\subsubsection{Electrical conductivity, density, permittivity and vis- cosity variations}

Finally, the effects of viscosity variation are included by considering the experimental viscosity ratio of 0.284 . Phenol is about 3.5 times more viscous than the 


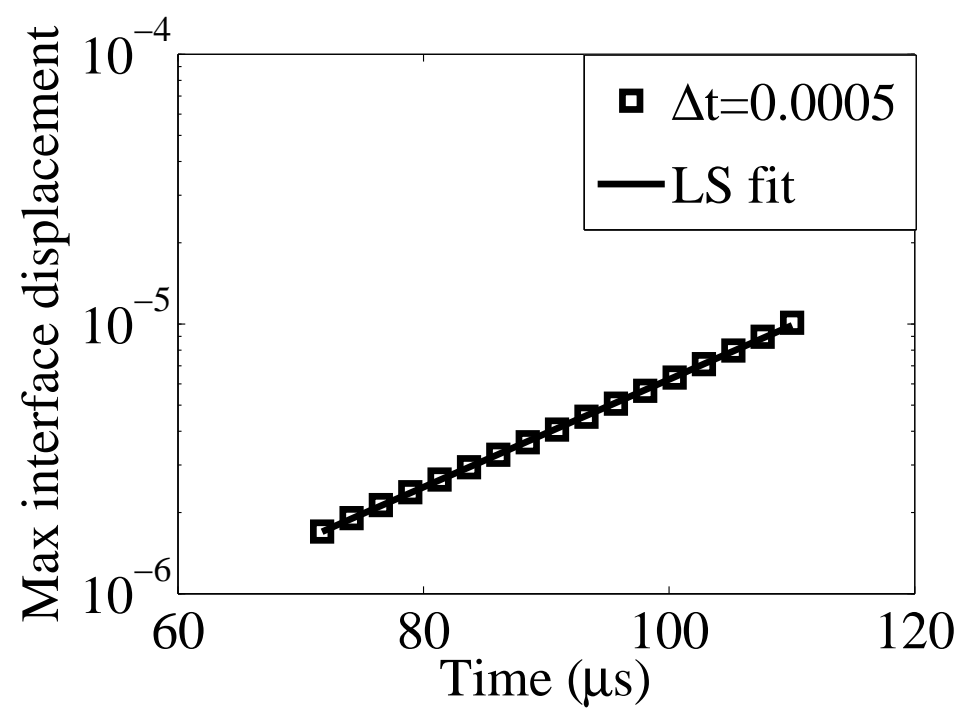

Figure 3.12: Growth rates extracted from nonlinear simulation for $k^{*}=10.0$, kink mode for a conductivity ratio 100 , density ratio 0.752 and permittivity ratio 10.974. The ' $\square$ ' are from the simulation with a dimensionless time step size of $\Delta t=5 \times 10^{-4}$. The extracted growth rate is $4.602 \times 10^{4} s^{-1}$, a $14.90 \%$ difference from the linear analysis result.

aqueous phase. This represents the complete set of property variations considered in the experiments of Zahn and Reddy [3], albeit with a lower conductivity ratio and negligible surface tension values due to the justification provided earlier. It is also worth mentioning that solving the Poisson's equation for indicator function with a very high conductivity ratio (for example, the experimental ratio of 5405) was found to be computationally expensive with the current solution methodology and highly efficient algorithms like Multigrid methods need to be implemented in such a situation. A detailed study and implementation of such algorithms is out of scope of the current work and will be considered in future works. The initial condition in this case is taken to be a sausage-mode disturbance with a dimensionless wavenumber of $k^{*}=10$, and a dimensionless amplitude 


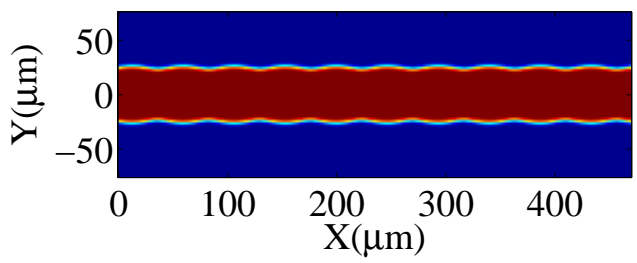

(a) $\mathrm{t}=75 \mu \mathrm{s}$.

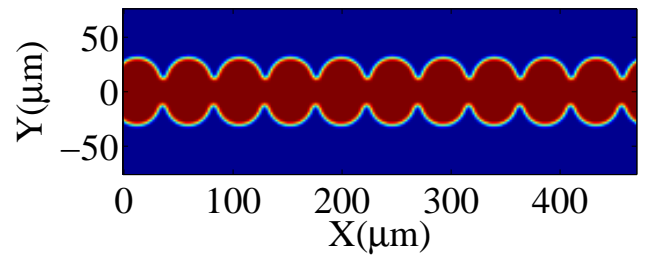

(b) $\mathrm{t}=150 \mu \mathrm{s}$.

Figure 3.13: Interface evolution for the case of conductivity ratio 100, density ratio 0.752 , permittivity ratio 10.974 and viscosity ratio 0.284 with an initial sausage-mode perturbation of $k^{*}=10.0$. The profiles at (a) $75 \mu \mathrm{s}$ and (b) 150 $\mu s$ are shown. Red denotes the high viscosity region (phenol), and blue, the low viscosity regions (aqueous phase).

of $5 \times 10^{-3}$. The viscosity profile evolution snapshots at two different instants of time are provided in figures 3.13. The electroviscous velocity scale for this case is calculated to be $1.11 \mathrm{~m} / \mathrm{s}$, time scale being $67.35 \mu \mathrm{s}$ and the Reynolds number is 32. The electroviscous time scale is higher than that for the case without viscosity variation and hence, we would expect the growth rate would be smaller. The growth rate from linear analysis for the sausage-mode disturbance is obtained as $3.014 \times 10^{4} s^{-1}$. From the nonlinear simulation data plotted in figure 3.3.4, the slope of the least-squares fit is calculated to be $3.306 \times 10^{4} s^{-1}$, a relative error of $9.7 \%$ compared to the linear analysis result, showing reasonable agreement with the linear analysis result. All the afore-mentioned simulation results pertain to an idealization with regard to the initial condition viz., biasing with either a low-amplitude kink or a sausage-mode perturbation. In order to simulate more realistically experimental conditions as in [3], we perturb the initially quiescent interface with a white Gaussian noise of dimensionless amplitude $6.67 \times 10^{-4}$ and 


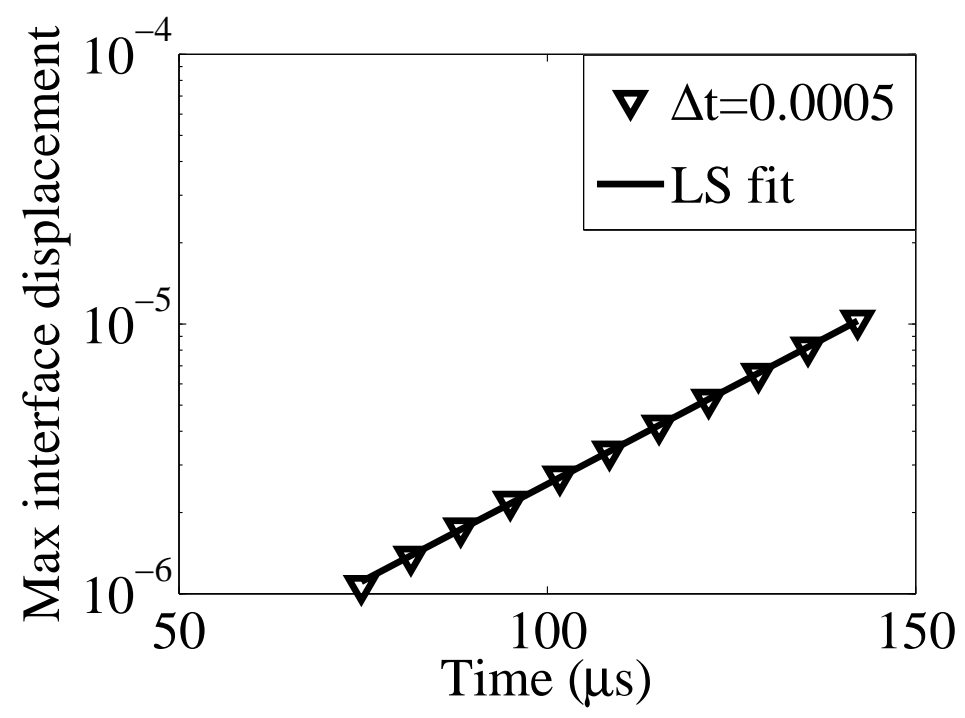

Figure 3.14: Growth rates extracted from nonlinear simulation for $k^{*}=10.0$, sausage mode for a conductivity ratio 100 , density ratio 0.752 , permittivity ratio 10.974 and viscosity ratio 0.284 . The ' $\nabla$ ' are from the simulation with a dimensionless time step size of $\Delta t=5 \times 10^{-4}$. The extracted growth rate is $3.298 \times 10^{4} s^{-1}$, a $9.70 \%$ difference from the linear analysis result.

provide some preliminary results showing the evolution of the interface fronts. The snapshots taken at $\mathrm{t}=270 \mu \mathrm{s}$ and $\mathrm{t}=320 \mu \mathrm{s}$ for conductivity are shown in figure 3.15. As can be seen from the figures, the resulting interfacial deformation exhibits mixed kink and sausage modes at different wavenumbers. It is well known that though the interface profile evolution exhibits mixed modes, there is a certain mode (kink/sausage) of suitable wavenumber which has the highest growth rate and is most prominently observed. To determine the fastest growth rate wavenumber and the corresponding mode, a Fourier-mode spectrum analysis on the white noise results needs to be performed after sufficient validation of comprehensive data sets. In this regard, addition of surface tension becomes important as surface tension sufficiently stabilizes the high wavenumbers. This 


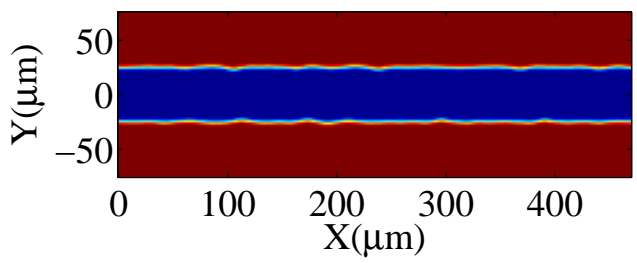

(a) $\mathrm{t}=270 \mu s$.

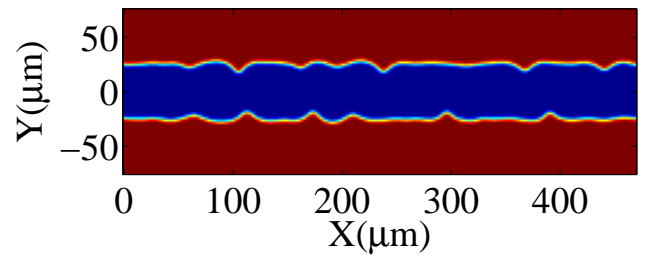

(b) $\mathrm{t}=320 \mu \mathrm{s}$.

Figure 3.15: Interface profile evolution snapshots at (a) $270 \mu \mathrm{s}$ and (b) $320 \mu \mathrm{s}$ for the interfaces initialized with small-amplitude white-noise disturbance and properties mentioned in figure 3.13. The profiles exhibit mixed unstable modes of different wavenumbers as observed in experiments of Zahn and Reddy [3].

\begin{tabular}{|c|c|c|c|}
\hline $\begin{array}{c}\text { Case } \\
(\text { Mode }- \text { Sausage }(\mathrm{S}) \text { or Kink }(\mathrm{K}))\end{array}$ & $\begin{array}{c}\text { Linear } \\
\left(s^{-1}\right)\end{array}$ & $\begin{array}{c}\text { Nonlinear } \\
\left(s^{-1}\right)\end{array}$ & $\begin{array}{c}\text { Rel. } \\
\text { error }(\%)\end{array}$ \\
\hline I $(\sigma=100(\mathrm{~S}))$ & $1.733 \times 10^{5}$ & $1.877 \times 10^{5}$ & 8.25 \\
\hline II $(\sigma=100, \rho=0.75(\mathrm{~S}))$ & $1.619 \times 10^{5}$ & $1.615 \times 10^{5}$ & 0.35 \\
\hline III $(\sigma=100, \rho=0.75, \epsilon=10.97(\mathrm{~K}))$ & $4.005 \times 10^{4}$ & $4.602 \times 10^{4}$ & 14.9 \\
\hline IV $(\sigma=100, \rho=0.75, \epsilon=10.97, \mu=0.28(\mathrm{~S}))$ & $3.014 \times 10^{4}$ & $3.306 \times 10^{4}$ & 9.7 \\
\hline
\end{tabular}

Table 3.1: Summary of growth rate results comparison between linear and nonlinear analyses for the four different cases.

has not yet been performed and would be conducted as part of future research. The comparison between linear and nonlinear analyses results for the various parametric ratios considered above have been summarized in table 3.1 .

\subsubsection{Grid resolution results}

It was mentioned earlier that the front-tracking algorithm employed for the simulations was second-order accurate with respect to grid resolution $\Delta x$. Though in-depth grid resolution related studies and validations are earmarked for future 
work, we provide some preliminary results for the case where all property variations are considered and the grid resolution is doubled. The initial condition is biased with a sausage-mode perturbation with a dimensionless wavenumber of $k^{*}=10$, and a dimensionless amplitude of $5 \times 10^{-3}$, so that a comparison of growth rate results with the original grid resolution is possible. Figure 3.16 depicts snapshots at $80 \mu s$ and $120 \mu s$ for conductivity and charge density profiles.

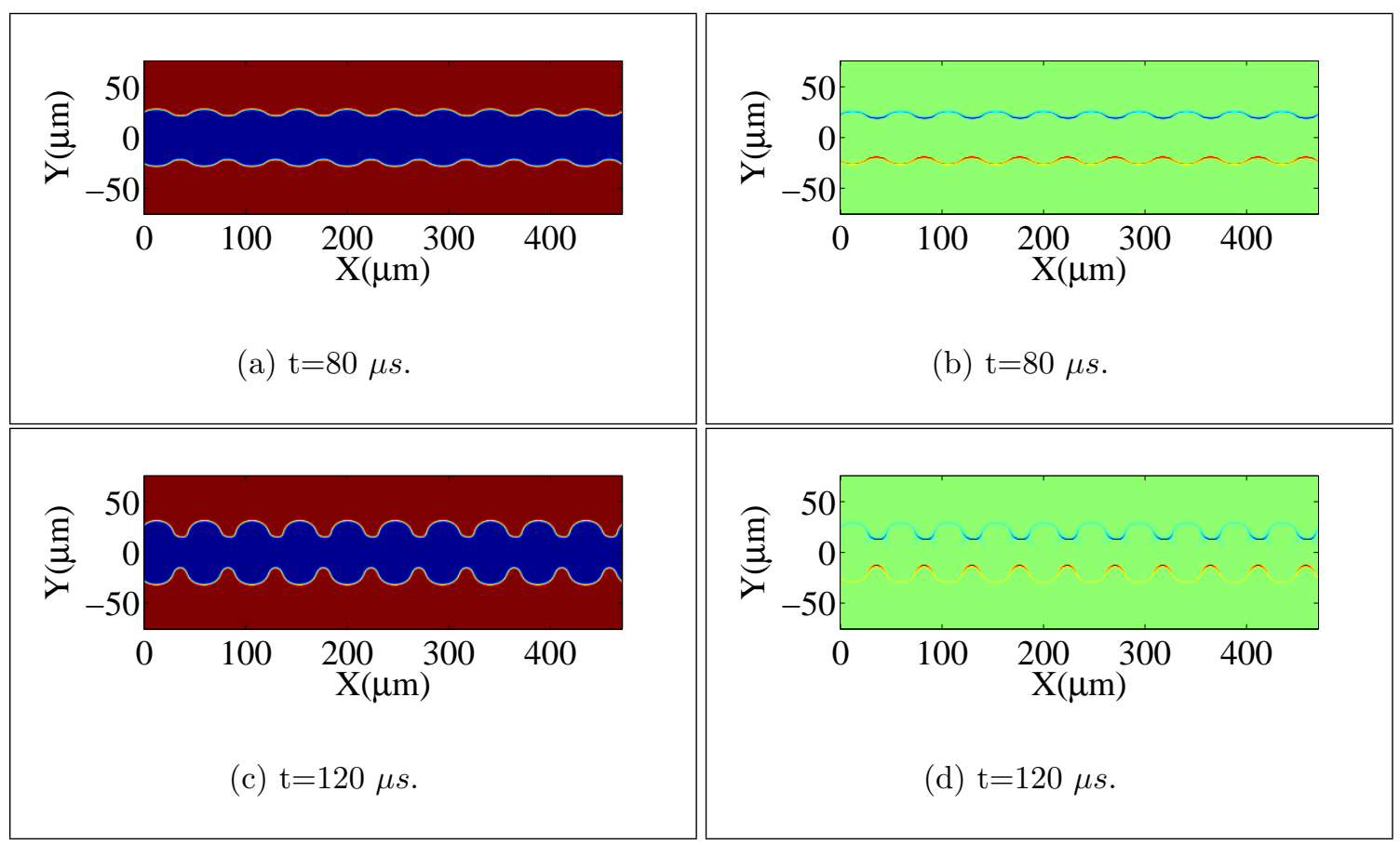

Figure 3.16: Interface profile evolution for the case of conductivity ratio of 100 , density ratio of 0.752 , permittivity ratio of 10.974 , viscosity ratio of 0.284 with an initial sausage-mode perturbation of $k^{*}=10.0$ and double the grid resolution compared to the previous cases. Conductivity and charge density snapshots at $80 \mu s$ are given in (a) and (b) respectively. Snapshots at $120 \mu s$ are provided in (c) and (d). 


\section{Chapter 4}

\section{Conclusions and directions of future work}

\subsection{Summary of the thesis}

Linear stability analysis and fully nonlinear simulations for two-dimensional electrohydrodynamic (EHD) instability in a three-layer viscous stratified (immiscible fluid) flow are presented. The motivation for the problem stems from the desired application of aqueous-organic (phenol) extraction technique in microfluidic platforms where efficient mixing of the two phases are limited by low Reynolds numbers. To overcome the shortcoming, electric fields are utilized to deform and ultimately rupture the fluid interfaces into droplets leading to efficient dispersion of one phase into another. The linear stability analysis was performed using Chebyshev pseudo-spectral method where growth rates corresponding to arbitrary wavenumbers were calculated by a solution of an eigenvalue problem. The results of the pseudo-spectral calculations specifically for the case of quiescent base flow were independently verified by solution using a root-finding methodology (not presented in this report). On the other hand, the methodology for nonlinear simulations is based on a front-tracking immersed boundary method to track interfacial deformation until the merging of the two interfaces. This tracking till 
interface merger is done fundamentally because a cogent multiphysics model to explain the progression from interface rupture to droplet formation is still a work in progress. In this thesis, our eventual objective is comparison of the growth rate results of "kink" (in-phase interfacial deformation) and "sausage" (interfacial deformations out of phase by $\pi$ ) modes from both linear and nonlinear analyses. The major results from the thesis are summarized as follows.

We outline the results from the linear stability analysis first. Based on the governing equations derived for the long-wave asymptotic analysis, it was determined that the electrical properties - electrical conductivity $\sigma$ and dielectric permittivity $\epsilon$ play the most crucial role in determining the stability/instability of the fluid flow. The contribution of the electrical properties is primarily through the terms $\left(Q_{T}\right)_{1},\left(Q_{T}\right)_{2},\left(Q_{N}\right)_{1}$ and $\left(Q_{N}\right)_{2}$ in tangential and normal shear stress balances at the two interfaces. Five different cases - A, B, C, D and E were considered based on the sign of the product " $(\sigma-1)\left(\sigma^{2}-\epsilon\right)$ ". The property ratios under the categories A and B (together called "Type-I") have a positive value for $(\sigma-1)\left(\sigma^{2}-\epsilon\right)$, those under the categories $\mathrm{C}$ and $\mathrm{D}$ (together named "Type-II") have a negative value and those under category E have a value of zero. We showed, for the cases of depth ratios " $\mathrm{n}$ " equaling 0.5 and 1 , that the perturbation growth rates are substantially higher for type-I compared to type-II meaning that a positive value leads to destabilizing effects and a negative value has stabilizing effects of the electric field. This finding was observed for both kink and the sausage modes and extends the results of EHD instability in a single 
interface between two immiscible fluids considered by Ozen et al. [23]. For the depth ratio $n=1$, a detailed log-log plot for a wide range $\left(10^{-2}\right.$ to $\left.10^{2}\right)$ of electrical properties combination exemplified the significant differences in characteristics of the maximum growth rates between kink and sausage modes. Next, we studied the effects of the electric field on growth rates and found that increase in electric field produces stabilizing or destabilizing effects based on the electrical property ratios. Overall for kink and sausage modes, with the increase in electric field, the flow was found to be stabilizing for case D with the contrary effect for case A. Subsequently, surface-tension effects were studied which revealed that with decrease in surface-tension, the growth rates for the short waves increased leading to a wider range of wavenumbers with the fastest growth rate. This clearly reflected the importance of surface tension in stabilizing short waves and resolving the fastest growth rate wavenumber. Finally, the effects of a convective base flow (plane Poiseuille) were considered. The results showed decrease in growth rates of the long waves and an increase in growth rates of the short waves leading to a conclusion that increase in the pressure gradient stabilized the long waves and destabilized the short waves. This behaviour was observed for both kink and sausage modes of cases A and D although the actual growth rate values were much higher for case A compared to case D (as expected from the earlier discussion).

We turn to the nonlinear simulation results next. The front tracking algorithm employed for the simulations was found to be first-order accurate in time. Since the immiscible fluids under consideration possess different mechanical (density 
and viscosity) and electrical (conductivity and permittivity) properties, it was necessary to introduce variations in sequence as a means of proving the robustness of the algorithm. The most important variation of electrical conductivity was considered first, followed by addition of density variation next, dielectric permittivity variation added subsequently and finally the viscosity ratio was included. The interfacial fronts and hence the relevant properties' profile evolution were tracked and plotted at several time instants for each of these cases. We temporarily ignore the surface tension effects and verified using the linear analysis that for the parametric ratios and wavenumbers considered in the simulation results, the neglection of surface tension results in an error of less than $3 \%$ in the growth rate calculation. The growth rate results were calculated from the slope of a least-squares fit of a semilog plot containing maximum interfacial deformation as the ordinate and time (in seconds) as the abscissa. The calculated growth rate results from nonlinear analysis for both kink and sausage modes provide reasonable to excellent agreement with those from linear analysis. The growth rates for the wavenumber considered $\left(k^{*}=10\right)$ were found to be the highest for the case with conductivity variation alone and decreased with a subsequent introduction of other property ratios, with the dielectric permittivity inclusion contributing to the most significant decrease. Lastly, exemplary results were provided for growth rate calculation considering all the property variations but with the resolution (grid points) doubled. It was shown that the growth rate calculation was in excellent agreement with that calculated using the original resolution data. 


\subsection{Future directions}

The need for efficient mixing of immiscible fluids in miniaturized microfluidic platforms has led to research studies aimed at control of droplet formation using external fields. The increase in attention has been fueled particularly due to the immense applications of mixing in chemical and biomedical fields like drug delivery systems, bio-purification systems to name a few. Interface deformation, rupture and subsequent droplet formation using electric fields in a microfluidic device is an extremely complex multiphase, multiscale problem and hence a comprehensive modeling of these processes is out of scope for a single thesis. This thesis is a preliminary work on stability analysis of generalized two phase microfluidic mixing using electric fields and therefore further research work is required. We address the future directions in the following paragraphs.

With regard to the linear analysis, the study of long-wave asymptotic behaviour would provide further invaluable insights into the contributions of electrical properties and depth ratio towards stabilization/destabilization of flow. The derivation of an analytical solution for long wave behaviour (governing equations provided in this thesis) in two-dimensional three layer viscous stratified flow would be taken up first and the results would be validated with the pseudospectral method. Simlar to the analytical expressions obtained for the single interface case in Li et al. [24], we expect detailed study of various important parameters like the depth ratio. For a quiescent base flow case for which this long wave analysis would not be valid, the "root-finding" methodology (not provided in this thesis) 
would be employed. Neutral stability curves for a wide range of depth ratios, electrical property ratios and electric fields (and hence, Capillary numbers) for both quiescent and plane Poiseuille base flows would be the eventual objective.

It was mentioned earlier that surface tension effects were neglected in the nonlinear simulations since the parametric ratios considered in the results yielded an error of less than $3 \%$ without its inclusion. Nonetheless, from the linear analysis it was noted that surface tension stabilizes short waves and hence needs to be included in the future for simulating kink and sausage modes for higher wavenumbers. The growth rate results for kink mode from the simulation data was found to be less accurate compared to those for sausage mode. The reasons for this observation need to be investigated. Furthermore, all the simulation results were for the case of a quiescent base flow and therefore plane Poiseuille base flow (with different pressure gradient values) results would need to be provided. Most importantly, it was stated that the algorithm was second-order accurate in space while validation of the algorithm for grid resolution independence was not provided. Even though growth rate results were in excellent agreement for original and double resolution cases, a noticeable difference in the maximum interfacial deformations was observed. Grid resolution studies and related discrepancies would be addressed in future works. Finally, parallelization and efficient numerical algorithms suitable for very high conductivity ratios would be implemented. 


\section{Appendix A}

\section{Long-wave asymptotic analysis}

A slightly different convention (refer to Chapter 2) is adopted here: the bottom interface is numbered 1 , its displacement denoted by $S_{1}(x, t)$ and the top interface is numbered 2 with its displacement denoted by $S_{2}(x, t)$.

\section{A.1 Scaling and dimensionless parameters}

We intend to provide the dimensionless forms of the governing equations (as in [24]), the solution of which would provide key understanding of the effects of various important dimensionless parameters on flow stability. The scales considered in the long-wave analysis are listed in table A.1 and the dimensionless ratios are given in equation (A.1.1):

$$
\begin{aligned}
& \mu=\frac{\mu_{1}}{\mu_{2}}, \rho=\frac{\rho_{1}}{\rho_{2}}, \sigma=\frac{\sigma_{1}}{\sigma_{2}}, \epsilon=\frac{\epsilon_{1}}{\epsilon_{2}},, \eta=2 n+1, n=\frac{a}{b}, \\
& C a=\frac{\mu_{2} U_{e v}}{\gamma}, R e_{j}=\frac{\rho_{j} U_{e v}(d)}{\mu_{2}}(j=1,2), \frac{R e_{2}}{R e_{1}}=\frac{1}{\rho},
\end{aligned}
$$

where, $U_{e v}$ is the electro-viscous velocity scale [12] representing the balance between electric body force and the viscous forces, $t_{e v}$ is the electro-viscous time scale, $n$ is the depth ratio and $\eta$ is the channel half-width, $C a$ is the capillary 


\begin{tabular}{|c|c|c|}
\hline Parameter & Scale & Scale description \\
\hline Length & $d=b / 2$ & Half width of sandwiched layer \\
\hline Velocity & $U_{e v}=\epsilon_{2} E_{0}^{2}(d) / \mu_{2}$ & Electro-viscous velocity \\
\hline Time & $t_{e v}=d / U_{e v}$ & Electro-viscous time \\
\hline Pressure & $\rho_{2} U_{e v}^{2}$ & \\
\hline Potential & $V_{0}$ & Lower wall electric potential \\
\hline Viscosity & $\mu_{2}$ & Viscosity of organic fluid \\
\hline Density & $\rho_{2}$ & Density of organic fluid \\
\hline Conductivity & $\sigma_{2}$ & Conductivity of organic fluid \\
\hline Permittivity & $\epsilon_{2}$ & Permittivity of organic fluid \\
\hline
\end{tabular}

Table A.1: Various scales used in the long-wave analysis.

number which is the ratio of the viscous force to the surface tension force and $R e$ is the Reynolds number. $\sigma, \epsilon, \rho$ and $\mu$ are the electrical conductivity, dielectric permittivity, density and viscosity ratios respectively.

\section{A.2 Dimensionless governing equations}

Firstly, the fluid flow variables i.e., velocities in $\mathrm{x}$ - and $\mathrm{y}$ - directions are considered. The continuity equation in each layer is given by:

$$
\frac{\partial u^{(j)}}{\partial x}+\frac{\partial v^{(j)}}{\partial y}=0, \quad(j=1,2,3)
$$

The $\mathrm{x}$ - and $\mathrm{y}$-momentum equations in each layer are given by:

$$
\begin{aligned}
& \frac{\partial u^{(1)}}{\partial t}+\mathbf{u}^{(1)} \cdot \nabla u^{(1)}=-\frac{1}{\rho} \frac{\partial p^{(1)}}{\partial x}+\frac{1}{R e_{1}}\left(\frac{\partial^{2} u^{(1)}}{\partial x^{2}}+\frac{\partial^{2} u^{(1)}}{\partial y^{2}}\right), 1 \leq \mathrm{y} \leq \eta \\
& \frac{\partial v^{(1)}}{\partial t}+\mathbf{u}^{(1)} \cdot \nabla v^{(1)}=-\frac{1}{\rho} \frac{\partial p^{(1)}}{\partial y}+\frac{1}{R e_{1}}\left(\frac{\partial^{2} v^{(1)}}{\partial x^{2}}+\frac{\partial^{2} v^{(1)}}{\partial y^{2}}\right), 1 \leq \mathrm{y} \leq \eta
\end{aligned}
$$




$$
\begin{gathered}
\frac{\partial u^{(2)}}{\partial t}+\mathbf{u}^{(2)} \cdot \nabla u^{(2)}=-\frac{\partial p^{(2)}}{\partial x}+\frac{1}{R e_{2}}\left(\frac{\partial^{2} u^{(2)}}{\partial x^{2}}+\frac{\partial^{2} u^{(2)}}{\partial y^{2}}\right),-1 \leq \mathrm{y} \leq 1 \\
\frac{\partial v^{(2)}}{\partial t}+\mathbf{u}^{(2)} \cdot \nabla v^{(2)}=-\frac{\partial p^{(2)}}{\partial y}+\frac{1}{R e_{2}}\left(\frac{\partial^{2} v^{(2)}}{\partial x^{2}}+\frac{\partial^{2} v^{(2)}}{\partial y^{2}}\right),-1 \leq \mathrm{y} \leq 1 \\
\frac{\partial u^{(3)}}{\partial t}+\mathbf{u}^{(3)} \cdot \nabla u^{(3)}=-\frac{1}{\rho} \frac{\partial p^{(3)}}{\partial x}+\frac{1}{R e_{1}}\left(\frac{\partial^{2} u^{(3)}}{\partial x^{2}}+\frac{\partial^{2} u^{(3)}}{\partial y^{2}}\right),-\eta \leq \mathrm{y} \leq-1 \\
\frac{\partial v^{(3)}}{\partial t}+\mathbf{u}^{(3)} \cdot \nabla v^{(3)}=-\frac{1}{\rho} \frac{\partial p^{(3)}}{\partial y}+\frac{1}{R e_{1}}\left(\frac{\partial^{2} v^{(3)}}{\partial x^{2}}+\frac{\partial^{2} v^{(3)}}{\partial y^{2}}\right),-\eta \leq \mathrm{y} \leq-1
\end{gathered}
$$

subject to the no-slip wall boundary conditions:

$$
u^{(3)}(-\eta)=0, v^{(3)}(-\eta)=0,
$$

at the lower wall and,

$$
u^{(1)}(\eta)=0, v^{(1)}(\eta)=0
$$

at the upper wall. Next, the conditions at the two interfaces need to be specified. The continuity of $\mathrm{x}$ - and $\mathrm{y}$ - velocity at the two interfaces require:

$$
\begin{gathered}
u^{(2)}=u^{(3)}, v^{(2)}=v^{(3)}, \quad \text { at } y=-1+S_{1}(x, t), \\
S_{1 t}+S_{1 x} u^{(j)}=v^{(j)}, \quad \text { at } y=-1+S_{1}(x, t),
\end{gathered}
$$

for the bottom interface where $\mathrm{j}=2,3$ and,

$$
\begin{gathered}
u^{(1)}=u^{(2)}, v^{(1)}=v^{(2)}, \quad \text { at } y=1+S_{2}(x, t), \\
S_{2 t}+S_{2 x} u^{(j)}=v^{(j)}, \quad \text { at } y=1+S_{2}(x, t),
\end{gathered}
$$


for the top interface where $\mathrm{j}=1,2$. Equations (A.2.2j) and (A.2.21) represent the kinematic constraints imposed on the lower and upper interfaces respectively. Secondly, the electrical field variables are considered. The electric potential in each layer satisfies the Laplace equation (2.2):

$$
\frac{\partial^{2} \Phi^{(j)}}{\partial x^{2}}+\frac{\partial^{2} \Phi^{(j)}}{\partial y^{2}}=0, \quad(j=1,2,3),
$$

subject to the potential wall boundary conditions:

$$
\Phi^{(3)}(-\eta)=1,
$$

for the applied electric potential at the lower wall and,

$$
\Phi^{(1)}(\eta)=0,
$$

for the grounded upper wall. The continuity of tangential electric field (2.2.5) entails:

$$
S_{1 x}\left(\Phi_{y}^{(3)}-\Phi_{y}^{(2)}\right)+\left(\Phi_{x}^{(3)}-\Phi_{x}^{(2)}\right)=0, \quad \text { at } y=-1+S_{1}(x, t),
$$

for the bottom interface and,

$$
S_{2 x}\left(\Phi_{y}^{(2)}-\Phi_{y}^{(1)}\right)+\left(\Phi_{x}^{(2)}-\Phi_{x}^{(1)}\right)=0, \quad \text { at } y=1+S_{2}(x, t),
$$


for the top interface. Similarly, the normal electric field continuity (2.2.6) can be expanded to obtain:

$$
S_{1 x}\left(\sigma \Phi_{x}^{(3)}-\Phi_{x}^{(2)}\right)=\left(\sigma \Phi_{y}^{(3)}-\Phi_{y}^{(2)}\right), \quad \text { at } y=-1+S_{1}(x, t),
$$

for the bottom interface and,

$$
S_{2 x}\left(\Phi_{x}^{(2)}-\sigma \Phi_{x}^{(1)}\right)=\left(\Phi_{y}^{(2)}-\sigma \Phi_{y}^{(1)}\right), \quad \text { at } y=1+S_{2}(x, t),
$$

for the top interface. Note that the subscripts in (A.2.3d)-(A.2.3g) denote the partial differentiation with respect to the relevant variable (x or y). Finally, the total stress continuity in the tangential and normal direction to the interfaces have to be satisfied. Substituting the expression for the total stress tensor (sum of fluid and electric stress tensors) in (2.2.4) into the tangential stress continuity equation (2.2.9) and expanding, it becomes:

$$
\begin{aligned}
& S_{1 x}\left\{2 \mu\left(\frac{\partial v^{(3)}}{\partial y}-\frac{\partial u^{(3)}}{\partial x}\right)-2\left(\frac{\partial v^{(2)}}{\partial y}-\frac{\partial u^{(2)}}{\partial x}\right)\right\} \\
& +\left(1-S_{1 x}^{2}\right)\left\{\mu\left(\frac{\partial u^{(3)}}{\partial y}+\frac{\partial v^{(3)}}{\partial x}\right)-\left(\frac{\partial u^{(2)}}{\partial y}+\frac{\partial v^{(2)}}{\partial x}\right)\right\} \\
& +S_{1 x}\left\{\epsilon\left(E_{2}^{(3) 2}-E_{1}^{(3) 2}\right)-\left(E_{2}^{(2) 2}-E_{1}^{(2) 2}\right)\right\} \\
& +\left(1-S_{1 x}^{2}\right)\left\{\epsilon E_{1}^{(3)} E_{2}^{(3)}-E_{1}^{(2)} E_{2}^{(2)}\right\}=0, \quad \text { at } y=-1+S_{1}(x, t),
\end{aligned}
$$


for the lower interface and:

$$
\begin{aligned}
& S_{2 x}\left\{2\left(\frac{\partial v^{(2)}}{\partial y}-\frac{\partial u^{(2)}}{\partial x}\right)-2 \mu\left(\frac{\partial v^{(1)}}{\partial y}-\frac{\partial u^{(1)}}{\partial x}\right)\right\} \\
& +\left(1-S_{2 x}^{2}\right)\left\{\left(\frac{\partial u^{(2)}}{\partial y}+\frac{\partial v^{(2)}}{\partial x}\right)-\mu\left(\frac{\partial u^{(1)}}{\partial y}+\frac{\partial v^{(1)}}{\partial x}\right)\right\} \\
& +S_{2 x}\left\{\left(E_{2}^{(2) 2}-E_{1}^{(2) 2}\right)-\epsilon\left(E_{2}^{(1) 2}-E_{1}^{(1) 2}\right)\right\} \\
& +\left(1-S_{2 x}^{2}\right)\left\{E_{1}^{(2)} E_{2}^{(2)}-\epsilon E_{1}^{(1)} E_{2}^{(1)}\right\}=0, \quad \text { at } y=1+S_{2}(x, t),
\end{aligned}
$$

for the upper interface. Proceeding similarly for the normal components of viscous and the electric stresses in (2.2.10), we obtain the normal stress continuity as:

$$
\begin{aligned}
& \left(1+S_{1 x}^{2}\right) R e_{2}\left(p_{2}-p_{3}\right)+2 \mu\left\{S_{1 x}^{2} \frac{\partial u^{(3)}}{\partial x}-S_{1 x}\left(\frac{\partial u^{(3)}}{\partial y}+\frac{\partial v^{(3)}}{\partial x}\right)+\frac{\partial v^{(3)}}{\partial y}\right\} \\
& -2\left\{S_{1 x}^{2} \frac{\partial u^{(2)}}{\partial x}-S_{1 x}\left(\frac{\partial u^{(2)}}{\partial y}+\frac{\partial v^{(2)}}{\partial x}\right)+\frac{\partial v^{(2)}}{\partial y}\right\} \\
& +\left(S_{1 x}^{2}-1\right)\left\{\frac{1}{2}\left(E_{2}^{(2) 2}-E_{1}^{(2) 2}\right)-\frac{\epsilon}{2}\left(E_{2}^{(3) 2}-E_{1}^{(3) 2}\right)\right\} \\
& -2 S_{1 x}\left\{\epsilon E_{1}^{(3)} E_{2}^{(3)}-E_{1}^{(2)} E_{2}^{(2)}\right\}-\frac{1}{C a} S_{1 x x}\left(1+S_{1 x}^{2}\right)^{-1 / 2}=0, \quad \text { at } y=-1+S_{1}(x, t)
\end{aligned}
$$

for the lower interface and:

$$
\begin{aligned}
& \left(1+S_{2 x}^{2}\right) R e_{2}\left(p_{1}-p_{2}\right)+2\left\{S_{2 x}^{2} \frac{\partial u^{(2)}}{\partial x}-S_{2 x}\left(\frac{\partial u^{(2)}}{\partial y}+\frac{\partial v^{(2)}}{\partial x}\right)+\frac{\partial v^{(2)}}{\partial y}\right\} \\
& -2 \mu\left\{S_{2 x}^{2} \frac{\partial u^{(1)}}{\partial x}-S_{2 x}\left(\frac{\partial u^{(1)}}{\partial y}+\frac{\partial v^{(1)}}{\partial x}\right)+\frac{\partial v^{(1)}}{\partial y}\right\} \\
& +\left(S_{2 x}^{2}-1\right)\left\{\frac{\epsilon}{2}\left(E_{2}^{(1) 2}-E_{1}^{(1) 2}\right)-\frac{1}{2}\left(E_{2}^{(2) 2}-E_{1}^{(2) 2}\right)\right\} \\
& -2 S_{2 x}\left\{E_{1}^{(2)} E_{2}^{(2)}-\epsilon E_{1}^{(1)} E_{2}^{(1)}\right\}-\frac{1}{C a} S_{2 x x}\left(1+S_{2 x}^{2}\right)^{-1 / 2}=0, \quad \text { at } y=1+S_{2}(x, t)
\end{aligned}
$$


for the upper interface. Note that $E_{1}$ and $E_{2}$ in the equations (A.2.4)-(A.2.7) denote the electric fields in $\mathrm{x}$ - and $\mathrm{y}$-directions respectively. Equations (A.2.1)(A.2.7) represent the complete set of dimensionless equations and relevant boundary conditions that fully specify the three-layer immiscible fluid EHD instability problem.

\section{A.3 Perturbation analysis}

We now perform the temporal stability analysis i.e. test the stability of the system subject to perturbations of the form $f^{\prime}(x, y, t)=\hat{f}(y) e^{(i k(x-c t))}$. " $f$ " can denote any variable (flow related or electrostatic). Here, $\hat{f}(y)$ represents the amplitude (complex), $\mathrm{k}$ is the wavenumber (real) and $\mathrm{c}\left(=c_{r}+i c_{i}\right)$ is the growth rate (complex) of the perturbation. $c_{r}$ and $c_{i}$ are the real and imaginary parts of the growth rate respectively. From the form of the perturbation, we can observe that the system is said to be stable if $c_{i}$ is negative and unstable if $c_{i}$ is positive. The bottom and top interfacial displacement amplitudes are denoted by $\hat{s}_{1}$ and $\hat{s}_{2}$ respectively.

We first derive the base state solution for the velocity and electric potential. The convention of representing the base state values using the subscript "0" will be followed unless mentioned otherwise. We assume a pressure-gradient driven convective base flow. By solving the continuity and Navier-Stokes equations with 
no-slip at the wall and velocity continuity at the interfaces, the velocity (dimensionless) in each layer can be obtained as:

$$
\begin{aligned}
& U_{0}^{(1)}=A_{1} y^{2}+b_{1}, \quad 1 \leq y \leq \eta, \\
& U_{0}^{(2)}=A_{2} y^{2}+b_{2}, \quad-1 \leq y \leq 1, \\
& U_{0}^{(3)}=A_{1} y^{2}+b_{1}, \quad-\eta \leq y \leq-1,
\end{aligned}
$$

where, $A_{1}=\left(R e_{2} / 2 \mu\right)(\partial p / \partial x), b_{1}=-A_{1} \eta^{2}, A_{2}=\mu A_{1}$ and $b_{2}=\left(1-\mu-\eta^{2}\right) A_{1}$. Note that $(\partial p / \partial x)$ is the base state dimensionless pressure gradient which is a constant and assumed to be known. The base state electric potential satisfies the Laplace's equation in each layer and combined with the tangential and normal electric field continuity at the interfaces, the solution for electric potential (dimensionless) in each layer can be expressed as:

$$
\begin{aligned}
& \Phi_{0}^{(1)}(y)=\frac{y-\eta}{2(1-\sigma-\eta)}, \quad 1 \leq y \leq \eta \\
& \Phi_{0}^{(2)}(y)=\frac{\sigma y}{2(1-\sigma-\eta)}+\frac{1}{2}, \quad-1 \leq y \leq 1, \\
& \Phi_{0}^{(3)}(y)=\frac{y+\eta}{2(1-\sigma-\eta)}+1, \quad-\eta \leq y \leq-1,
\end{aligned}
$$

For the interfaces in equilibrium, the dimensionless normal stress balance at the 
top and bottom interfaces yield:

$$
\begin{gathered}
\operatorname{Re}_{2}\left(p_{0}^{(1)}-p_{0}^{(2)}\right)+\frac{\eta^{2}}{2} \frac{\left(\sigma^{2}-\epsilon\right)}{(1-\sigma-\eta)^{2}}=0, \quad \text { at } y=1, \\
\operatorname{Re}_{2}\left(p_{0}^{(2)}-p_{0}^{(3)}\right)+\frac{\eta^{2}}{2} \frac{\left(\epsilon-\sigma^{2}\right)}{(1-\sigma-\eta)^{2}}=0, \quad \text { at } y=-1,
\end{gathered}
$$

respectively. Having obtained the base state profiles, we now proceed to the linear analysis. Considering the flow field first, we linearize the Navier-Stokes equations using $u^{(j)}=U_{0}^{(j)}+u^{(j)}, v^{(j)}=v^{(j)}, p^{(j)}=p_{0}^{(j)}+p^{(j)}(\mathrm{j}=1,2,3)$, where the primes indicate perturbation variables. We utilize streamfunction " $\psi$ " instead of primitive variables (x- and y-velocities), following the approach of Yih [4] and Li et al. [24]. The velocity perturbations are related to their streamfunction counterparts as $u^{(j)}=\partial \psi^{(j)} / \partial y, v^{(j)}=-\partial \psi^{(j)} / \partial x$, where $\psi^{(j)}(x, y, t)=\phi^{(j)} \exp (i k(x-c t))$ with $\phi^{(j)}$ being the complex amplitude. Substituting the total velocities and pressure into the Navier-Stokes equations (A.2.2a)-(A.2.2f), and neglecting second-order terms and higher, we obtain the classical Orr-Sommerfeld equation for each fluid layer by combining the expressions for $\mathrm{x}$ - and $\mathrm{y}$ - momentum equations:

$$
\begin{aligned}
& \left(\frac{d^{2}}{d y^{2}}-k^{2}\right)^{2} \phi^{(1)}=i k \operatorname{Re}_{1}\left[\left(U_{0}^{(1)}-c\right)\left(\frac{d^{2}}{d y^{2}}-k^{2}\right) \phi^{(1)}-\phi^{(1)} \frac{d^{2} U_{0}^{(1)}}{d y^{2}}\right], \\
& \left(\frac{d^{2}}{d y^{2}}-k^{2}\right)^{2} \phi^{(2)}=i k \operatorname{Re}_{2}\left[\left(U_{0}^{(2)}-c\right)\left(\frac{d^{2}}{d y^{2}}-k^{2}\right) \phi^{(2)}-\phi^{(2)} \frac{d^{2} U_{0}^{(2)}}{d y^{2}}\right], \\
& \left(\frac{d^{2}}{d y^{2}}-k^{2}\right)^{2} \phi^{(3)}=i k \operatorname{Re}_{1}\left[\left(U_{0}^{(3)}-c\right)\left(\frac{d^{2}}{d y^{2}}-k^{2}\right) \phi^{(3)}-\phi^{(3)} \frac{d^{2} U_{0}^{(3)}}{d y^{2}}\right],
\end{aligned}
$$


subject to boundary conditions (A.2.2g)-(A.2.2l) rewritten in terms of the streamfunction as:

$$
\begin{gathered}
\phi^{(3)}(-\eta)=0, \frac{d \phi^{(3)}}{d y}(-\eta)=0, \\
\phi^{(1)}(\eta)=0, \frac{d \phi^{(1)}}{d y}(\eta)=0, \\
\phi^{(2)}=\phi^{(3)}=\phi(-1), \frac{d \phi^{(3)}}{d y}(-1)-\frac{d \phi^{(2)}}{d y}(-1)=\frac{2 \phi(-1)}{c_{I}^{\prime}}\left(A_{1}-A_{2}\right), \\
S_{1}=\frac{\phi(-1)}{c_{I}^{\prime}} \exp (i k(x-c t)), \\
\phi^{(1)}=\phi^{(2)}=\phi(1), \frac{d \phi^{(2)}}{d y}(1)-\frac{d \phi^{(1)}}{d y}(1)=\frac{2 \phi(1)}{c_{I I}^{\prime}}\left(A_{1}-A_{2}\right), \\
S_{2}=\frac{\phi(1)}{c_{I I}^{\prime}} \exp (i k(x-c t)),
\end{gathered}
$$

where, $c_{I I}^{\prime}=c-U_{0}^{(1)}(1)=c-U_{0}^{(2)}(1)$ and $c_{I}^{\prime}=c-U_{0}^{(2)}(-1)=c-U_{0}^{(3)}(-1)$. Proceeding to the electric field related effects, we denote the total electric potential as $\Phi^{(j)}=\Phi_{0}^{(j)}+\chi^{(j)}(\mathrm{j}=1,2,3)$, where $\chi^{(j)}=\hat{\chi}(y) \exp (i k(x-c t))$ is the electric potential perturbation. Substituting the total potential into (A.2.3a)-(A.2.3g), and cancelling out base-state terms, the governing equations for the potential perturbations are:

$$
\frac{\partial^{2} \chi^{(j)}}{\partial x^{2}}+\frac{\partial^{2} \chi^{(j)}}{\partial y^{2}}=0, \quad(j=1,2,3)
$$

subject to the potential wall boundary conditions:

$$
\chi^{(3)}(-\eta)=0
$$




$$
\chi^{(1)}(\eta)=0,
$$

and interfacial continuity conditions:

$$
\begin{gathered}
S_{1 x} \frac{(1-\sigma)}{2(1-\sigma-\eta)}+\left(\chi_{x}^{(3)}-\chi_{x}^{(2)}\right)=0, \quad \text { at } y=-1, \\
S_{2 x} \frac{(\sigma-1)}{2(1-\sigma-\eta)}+\left(\chi_{x}^{(2)}-\chi_{x}^{(1)}\right)=0, \quad \text { at } y=1, \\
\chi_{y}^{(2)}=\sigma \chi_{y}^{(3)}, \quad \text { at } y=-1, \\
\sigma \chi_{y}^{(1)}=\chi_{y}^{(2)}, \quad \text { at } y=1,
\end{gathered}
$$

Equations (A.3.5a)-(A.3.5g) can be solved straightforwardly to obtain the potential perturbations in each layer as:

$$
\begin{aligned}
& \hat{\chi}^{(1)}=\frac{(\sigma-1) \sinh (\{\eta-y\} k)}{4(1-\sigma-\eta) \cosh (\{\eta-1\} k)}\left[K_{1}\left(\hat{s}_{1}+\hat{s}_{2}\right) s h-M_{1}\left(\hat{s}_{1}-\hat{s}_{2}\right) c h\right], \\
& \hat{\chi}^{(2)}=\frac{-\sigma(\sigma-1)}{4(1-\sigma-\eta)}\left[K_{1}\left(\hat{s}_{1}+\hat{s}_{2}\right) \cosh (k y)-M_{1}\left(\hat{s}_{1}-\hat{s}_{2}\right) \sinh (k y)\right], \\
& \hat{\chi}^{(3)}=\frac{(\sigma-1) \sinh (\{y+\eta\} k)}{4(1-\sigma-\eta) \cosh (\{\eta-1\} k)}\left[K_{1}\left(\hat{s}_{1}+\hat{s}_{2}\right) s h+M_{1}\left(\hat{s}_{1}-\hat{s}_{2}\right) c h\right],
\end{aligned}
$$

where, $K_{1}=(\operatorname{thsh}+\sigma c h)^{-1}, M_{1}=(t h c h+\sigma s h)^{-1}, \operatorname{th}=\tanh (\{\eta-1\} k)$, $s h=\sinh (k)$ and $c h=\cosh (k)$. Note that $\sinh$ and $\cosh$ are the hyperbolic sine and cosine functions respectively. Finally, substituting the total velocities and electric potential into the tangential and normal stress balance at the two 
interfaces in equations (A.2.4)-(A.2.7), and neglecting terms second-order and higher, we obtain the final forms as:

$$
\left[\mu\left(\frac{d^{2}}{d y^{2}}+k^{2}\right) \phi^{(3)}(-1)-\left(\frac{d^{2}}{d y^{2}}+k^{2}\right) \phi^{(2)}(-1)\right]+i k \eta^{2}\left(Q_{T}\right)_{1}=0
$$

for the lower interface, where, $\left(Q_{T}\right)_{1}$ represents the contribution of the electric stresses to the tangential stress continuity at top interface and is expressed as:

$$
\begin{aligned}
\left(Q_{T}\right)_{1} & =\frac{\hat{s}_{1}\left(\epsilon-\sigma^{2}\right)}{(1-\sigma-\eta)^{2}}+\frac{(\sigma-1)}{2(1-\sigma-\eta)^{2}}\left\{\frac{\left(\epsilon t h s h+\sigma^{2} c h\right)}{t h s h+\sigma c h}\left(\hat{s}_{1}+\hat{s}_{2}\right)\right\} \\
& +\frac{(\sigma-1)}{2(1-\sigma-\eta)^{2}}\left\{\frac{\left(\epsilon t h c h+\sigma^{2} s h\right)}{t h c h+\sigma s h}\left(\hat{s}_{1}-\hat{s}_{2}\right)\right\}
\end{aligned}
$$

and:

$$
\left[\left(\frac{d^{2}}{d y^{2}}+k^{2}\right) \phi^{(2)}(1)-\mu\left(\frac{d^{2}}{d y^{2}}+k^{2}\right) \phi^{(1)}(1)\right]+i k \eta^{2}\left(Q_{T}\right)_{2}=0,
$$

for the upper interface, where, $\left(Q_{T}\right)_{2}$ represents the contribution of the electric stresses to the tangential stress continuity at bottom interface and is expressed as:

$$
\begin{aligned}
\left(Q_{T}\right)_{2} & =\frac{\hat{s}_{2}\left(\sigma^{2}-\epsilon\right)}{(1-\sigma-\eta)^{2}}-\frac{(\sigma-1)}{2(1-\sigma-\eta)^{2}}\left\{\frac{\left(\epsilon t h s h+\sigma^{2} c h\right)}{t h s h+\sigma c h}\left(\hat{s}_{1}+\hat{s}_{2}\right)\right\} \\
& +\frac{(\sigma-1)}{2(1-\sigma-\eta)^{2}}\left\{\frac{\left(\epsilon t h c h+\sigma^{2} s h\right)}{t h c h+\sigma s h}\left(\hat{s}_{1}-\hat{s}_{2}\right)\right\}
\end{aligned}
$$

Proceeding similarly for the normal stress continuity, the final forms are: 


$$
\begin{aligned}
& {\left[\mu\left(\frac{d^{2}}{d y^{2}}-3 k^{2}\right) \frac{d \phi^{(3)}}{d y}(-1)-\left(\frac{d^{2}}{d y^{2}}-3 k^{2}\right) \frac{d \phi^{(2)}}{d y}(-1)\right]+i k\left[\rho\left(Q_{0}\right)_{1}-\frac{k^{2} \hat{s}_{1}}{C a}\right]} \\
& -i k \eta^{2}\left(Q_{N}\right)_{1}=0,
\end{aligned}
$$

for the lower interface, where, in addition to the electric stress contribution $\left(Q_{N}\right)_{1}$, we also have the contribution of the pressure terms $\left(Q_{0}\right)_{1}$ to the normal stress continuity at the top interface. The expressions for $\left(Q_{N}\right)_{1}$ and $\left(Q_{0}\right)_{1}$ are given by:

$$
\begin{aligned}
\left(Q_{N}\right)_{1} & =\frac{\left(\epsilon-\sigma^{2}\right)(\sigma-1) k}{2(1-\sigma-\eta)^{2}}\left\{\frac{s h}{t h s h+\sigma c h}\left(\hat{s}_{1}+\hat{s}_{2}\right)\right\} \\
& +\frac{\left(\epsilon-\sigma^{2}\right)(\sigma-1) k}{2(1-\sigma-\eta)^{2}}\left\{\frac{c h}{t h c h+\sigma s h}\left(\hat{s}_{1}-\hat{s}_{2}\right)\right\}, \\
\left(Q_{0}\right)_{1} & =-\operatorname{Re}_{2}\left[\left(c-U^{(3)}(-1)\right)\left(\frac{1}{\rho} \frac{d \phi^{(2)}}{d y}-\frac{d \phi^{(3)}}{d y}\right)+2 \phi(-1)\left(A_{1}-\frac{A_{2}}{\rho}\right)\right],
\end{aligned}
$$

and:

$$
\begin{aligned}
& {\left[\mu\left(\frac{d^{2}}{d y^{2}}-3 k^{2}\right) \frac{d \phi^{(2)}}{d y}(1)-\left(\frac{d^{2}}{d y^{2}}-3 k^{2}\right) \frac{d \phi^{(1)}}{d y}(1)\right]+i k\left[\rho\left(Q_{0}\right)_{2}-\frac{k^{2} \hat{\zeta}_{1}}{C a}\right]} \\
& -i k \eta^{2}\left(Q_{N}\right)_{2}=0,
\end{aligned}
$$

for the upper interface, where, $\left(Q_{0}\right)_{2}$ and $\left(Q_{N}\right)_{2}$ represent the contributions of the pressure terms and electric stresses to the normal stress continuity at bottom interface and are given as: 


$$
\begin{aligned}
\left(Q_{N}\right)_{2} & =\frac{\left(\epsilon-\sigma^{2}\right)(\sigma-1) k}{2(1-\sigma-\eta)^{2}}\left\{\frac{s h}{\text { thsh }+\sigma c h}\left(\hat{s}_{1}+\hat{s}_{2}\right)\right\} \\
& -\frac{\left(\epsilon-\sigma^{2}\right)(\sigma-1) k}{2(1-\sigma-\eta)^{2}}\left\{\frac{c h}{\text { thch }+\sigma s h}\left(\hat{s}_{1}-\hat{s}_{2}\right)\right\}, \\
\left(Q_{0}\right)_{2} & =-\operatorname{Re}_{2}\left[\left(c-U^{(2)}(1)\right)\left(\frac{d \phi^{(1)}}{d y}-\frac{1}{\rho} \frac{d \phi^{(2)}}{d y}\right)+2 \phi(1)\left(A_{1}-\frac{A_{2}}{\rho}\right)\right],
\end{aligned}
$$

It is worth mentioning that $\left(Q_{0}\right)_{1}$ and $\left(Q_{0}\right)_{2}$ would also contain contributions from body force terms (for example, gravity as considered in Li et al. [24]) considered in the fluid momentum equations. Since we neglect gravity, such a contribution is absent in those expressions. As discussed in Li et al. [24], we verify our results for the two limiting cases of (1) perfect dielectrics and (2) perfectly conducting surface where one fluid is highly conductive compared to the other. Substituting $\epsilon=\sigma$ for the case of perfect dielectrics in the expressions of $\left(Q_{T}\right)_{1}$ and $\left(Q_{T}\right)_{2}$, we get $\left(Q_{T}\right)_{1}=\left(Q_{T}\right)_{2}=0$, thereby recovering the result that perfect dielectrics do produce electrical normal stresses alone and no electrical tangential shear stresses. Subsequently, as $\sigma \rightarrow \infty$, we obtain $\left(Q_{T}\right)_{1} \rightarrow 0$ and $\left(Q_{T}\right)_{2} \rightarrow 0$ thereby verifying that electrical tangential stresses vanish at perfectly conducting interfaces. It can also be observed that when $\epsilon=\sigma^{2},\left(Q_{N}\right)_{1}=\left(Q_{N}\right)_{2}=0$ while $\left(Q_{T}\right)_{1} \neq 0$ and $\left(Q_{N}\right)_{2} \neq 0$, implying that electrical tangential stresses do not vanish and hence would be fundamentally responsible for deciding the flow stability for this case. This observation is also in agreement with that from Li et al. [24]. 


\section{References}

[1] R.J. HUNTER. Foundations of Colloid Science. Oxford University Press, 1989 .

[2] J. SAMBROOK, E.F. FRITSCH, and T. MANIATIS. Molecular cloning: a laboratory manual, vol 1-3. Cold Spring Harbor Laboratory Press, 1989.

[3] J.D. ZAHN, and V. REDDY. Two phase micromixing and analysis using electrohydrodynamic instabilities. Microfluid Nanofluid, 2:399-415, 2006.

[4] CHIA-SHUN YIH. Instability due to viscosity stratification. J. Fluid Mech., 27:337-352, 1967.

[5] CHIA-SHUN YIH. Stability of two dimensional parallel flows for three dimensional disturbances. Quart. Appl. Math., 12:434-435, 1955.

[6] CHIN-HSIU LI. Instability of three-layer viscous stratified flow. Physics of Fluids, 12:2473-2481, 1969.

[7] A.P. HOOPER, and W.G.C. BOYD. Shear-flow instability at the interface between two viscous fluids. J. Fluid Mech., 128:507-528, 1983.

[8] T.W. KAO, and C. PARK. Experimental investigation of the stability of channel flow. Part-2. Two-layered co-current flow in a rectangular channel. J. Fluid Mech., 52:401-423, 1972.

[9] S.G. YIANTSIOS, and B.G. HIGGINS. Linear stability of plane Poiseuille flow of two superposed fluids. Physics of Fluids, 11:3225-3238, 1988.

[10] M.H. ODDY, J.G. SANTIAGO, and J.C. MIKKELSEN. Electrokinetic instability micromixing. Anal Chem, 73:5822-5832, 2001.

[11] I. GLASGOW, J. BATTON, and N. AUBRY. Electroosmotic mixing in microchannels. Lab Chip, 4:558-562, 2004.

[12] H. LIN, B.D. STOREY, M.H. ODDY, C.H. CHEN, and J.G. SANTIAGO. Instability of electrokinetic microchannel flows with conductivity gradients. Physics of Fluids, 16:1922-1935, 2004.

[13] J.C. BAYGENTS and F. BALDESSARI. Electrohydrodynamic instability in a thin fluid layer with an electrical conductivity gradient. Physics of Fluids, 10:301, 1998. 
[14] C.H. CHEN, H. LIN, S.K. LELE and J.G. SANTIAGO. Convective and absolute electrokinetic instability with conductivity gradients. J. Fluid Mech., 524:263-303, 2005.

[15] O. OZEN, N. AUBRY, D.T. PAPAGEORGIOU, and P.G. PETROPOULOS. Monodisperse drop formation in square microchannels. Physical Review Letters, 96:144501, 2006.

[16] G.I. TAYLOR. Studies in electrohydrodynamics I. The circulation produced in a drop by an electric field. Proc. $R$. Soc. A, 291:159-66, 1966.

[17] J.R. MELCHER, and W.J. SCHWARZ. Interfacial relaxation overstability in a tangential electric field. Physics of Fluids, 11:2604, 1968.

[18] J.R. MELCHER, and C.V. SMITH. Electrohydrodynamic charge relaxation and interfacial perpendicular-field instability. Physics of Fluids, 12:778, 1969.

[19] J.F. HOBURG, and J.R. MELCHER. Internal electrohydrodynamic instability and mixing of fluids with orthogonal field and conductivity gradients. J. Fluid Mech., 73:333-351, 1976.

[20] A.A. MOHAMED, E.F. ELSHEHAWEY, and M.F. EL-SAYED. Electrohydrodynamic stability of two superposed viscous fluids. J. Colloid Interface Sci., 169:65-78, 1994.

[21] K. ABDELLA and H. RASMUSSEN. Electrohydrodynamic instability of two superposed fluids in normal electric fields. J. Computational and Applied Math., 78:33-61, 1997.

[22] D.D. JOSEPH, and Y. RENARDY. Fundamentals of two-fluid dynamics, Part I:Mathematical Theory and Applications. Springer, 1993.

[23] O. OZEN, N. AUBRY, D.T. PAPAGEORGIOU, and P.G. PETROPOULOS. Electrohydrodynamic linear stability of two immiscible fluids in channel flow. Electrochimica Acta, 51:5316-5323, 2006.

[24] F. LI, O. OZEN, N. AUBRY, D.T. PAPAGEORGIOU, and P.G. PETROPOULOS. Linear stability of a two-fluid interface for electrohydrodynamic mixing in a channel. Physical Review Letters, 583:347-377, 2007.

[25] A.K. UGUZ, O. OZEN, and N. AUBRY. Electric field effect on a two-fluid interface instability in channel flow for fast electric times. Physics of Fluids, 20:031702, 2008.

[26] D.A. SAVILLE. Electrohydrodynamics: The Taylor-Melcher Leaky Dielectric model. Annu. Rev. Fluid Mech., 29:27-64, 1997.

[27] J. SHERWOOD. Breakup of fluid droplets in electric and magnetic fields. J. Fluid Mech., 188:133-146, 1988. 
[28] J.W. HA and S.M. YANG. Effect of non-ionic surfactant on the deformation and breakup of a drop in an electric field. J. Colloid Interface Sci., 206:195204, 1998.

[29] J.C. BAYGENTS, N.J. RIVETTE and H.A. STONE. Electrohydrodynamic deformation and interaction of drop pairs. J. Fluid Mech., 188:133-146, 1988.

[30] M.T. HARRIS and A. BASARAN. Axisymmetric shapes and stability of pendant and sessile drops in an electric field. J. Colloid Interface Sci., 140(1):10-30, 1990.

[31] M.T. HARRIS and A. BASARAN. Capillary electrohydrostatics of conducting drops hanging from a nozzle in an electric field. J. Colloid Interface Sci., 170:308-319, 1995.

[32] M.T. HARRIS and A. BASARAN. Equilibrium shapes and stability of nonconducting pendant drops surrounded by a conducting fluid in an electric field. J. Colloid Interface Sci., 170:308-319, 1995.

[33] P.K. NOTZ and A. BASARAN. Dynamics of drop formation in an electric field. J. Colloid Interface Sci., 213:218-237, 1999.

[34] T. TSUKUDA, T. KATAYAMA, Y. ITO, and M. HOZAWA. Theoretical and experimental studies of circulations inside and outside a deformed drop under a uniform electric field. J. Chem. Eng. Jpn., 26:698, 1993.

[35] T. TSUKUDA, Y. YAMAMOTO, T. KATAYAMA and M. HOZAWA. Effect of an electric field on the behavior of a drop moving in a quiescent liquid. $J$. Chem. Eng. Jpn., 27:662, 1994.

[36] ARTURO FERNANDEZ, GRETAR TRYGGVASON, JUDY CHE and STEVEN L. CECCIO. The effects of electrostatic forces on the distribution of drops in a channel flow: Two-dimensional oblate drops. J. Colloid Interface Sci., 161:389-413, 1993.

[37] G. TRYGGVASON, B. BUnNER, A. ESMAEELI, D. JURIC, N. ALRAWAHI, W. TAUBER, J. HAN, S. NAS, and Y.J. JAN. A front-tracking method for the computations of multiphase flows. J. Comp. Phys., 169:708$759,2001$.

[38] S.O. UNVERDI and G.TRYGGVASON. A front-tracking method for viscous, incompressible, multi-fluid flows. J. Comp. Phys., 100:25-37, 1992.

[39] C.S. PESKIN. Flow patterns around heart valves: A numerical method. J. Comp. Phys., 25:220, 1977.

[40] C.S. PESKIN and D.M. MCQUEEN. A three-dimensional computational method for blood flow in the heart. J. Comp. Phys., 81:372, 1989. 
[41] A.J. CHORIN. Numerical Solution of the Navier-Stokes Equations. Math. Comp., 22:745-762, 1968.

[42] V.G. FERREIRA, M.F. TOME', N. MANGIAVACCHI, A. CASTELO, J.A. CUMINATO, A.O. FORTUNA and S. MCKEE. High-order upwinding and the hydraulic jump. Int. J. Numer. Meth. Fluids, 39:549-583, 2002.

[43] B.P. LEONARD. A stable and accurate convective modelling procedure based on quadratic upstream interpolation. Comp. Meth. Appl. Mech. Eng., 19:5998, 1979.

[44] P.H. GASKELL and A.K.C. LAU. Curvature-compensated convective transport: SMART, a new boundedness-preserving transport algorithm. Int. J. Num. Meth. Fluids, 8:617 641, 1988.

[45] W. TAUBER, S.O. UNVERDI, and G. TRYGGVASON. The nonlinear behavior of a sheared immiscible fluid interface. Physics of Fluids, 14:2871$2885,2002$.

[46] J. ZHANG and D.Y. KWOK. A 2D lattice Boltzmann study on electrohydrodynamic drop deformation with the leaky dielectric theory. J. Comput. Phys., 206:150-161, 2005.

[47] G. TOMAR, D. GERLACH, G. BISWAS, N. ALLEBORN, A. SHARMA, F. DURST, S.W.J. WELCH, and A. DELGADO. Two-phase electrohydrodynamic simulations using a volume-of-fluid approach. J. Comput. Phys., 227:1267-1285, 2007.

[48] J.P. BOYD. Chebyshev and Fourier Spectral Methods . Dover Publications Inc., 2001.

[49] R. PEYRET. Spectral methods for incompressible viscous flow. SpringerVerlag New York Inc., 2002.

[50] L.N. TREFETHEN. Spectral methods in MATLAB. Society for industrial and applied mathematics, 2000.

[51] J.A.C. Wiedemann and S.C. REDDY. A MATLAB differentiation matrix suite. ACM TOMS, 26:465-519, 2000. 